\begin{tabular}{lc}
\hline IFA $-95 / 29$ & July 1995 \\
\hline AARHUS-ASTRO-1995-17 & ISSN 0906-3870
\end{tabular}

\title{
TESTING A SOLAR MODEL: THE FORWARD PROBLEM
}

Jørgen Christensen-Dalsgaard
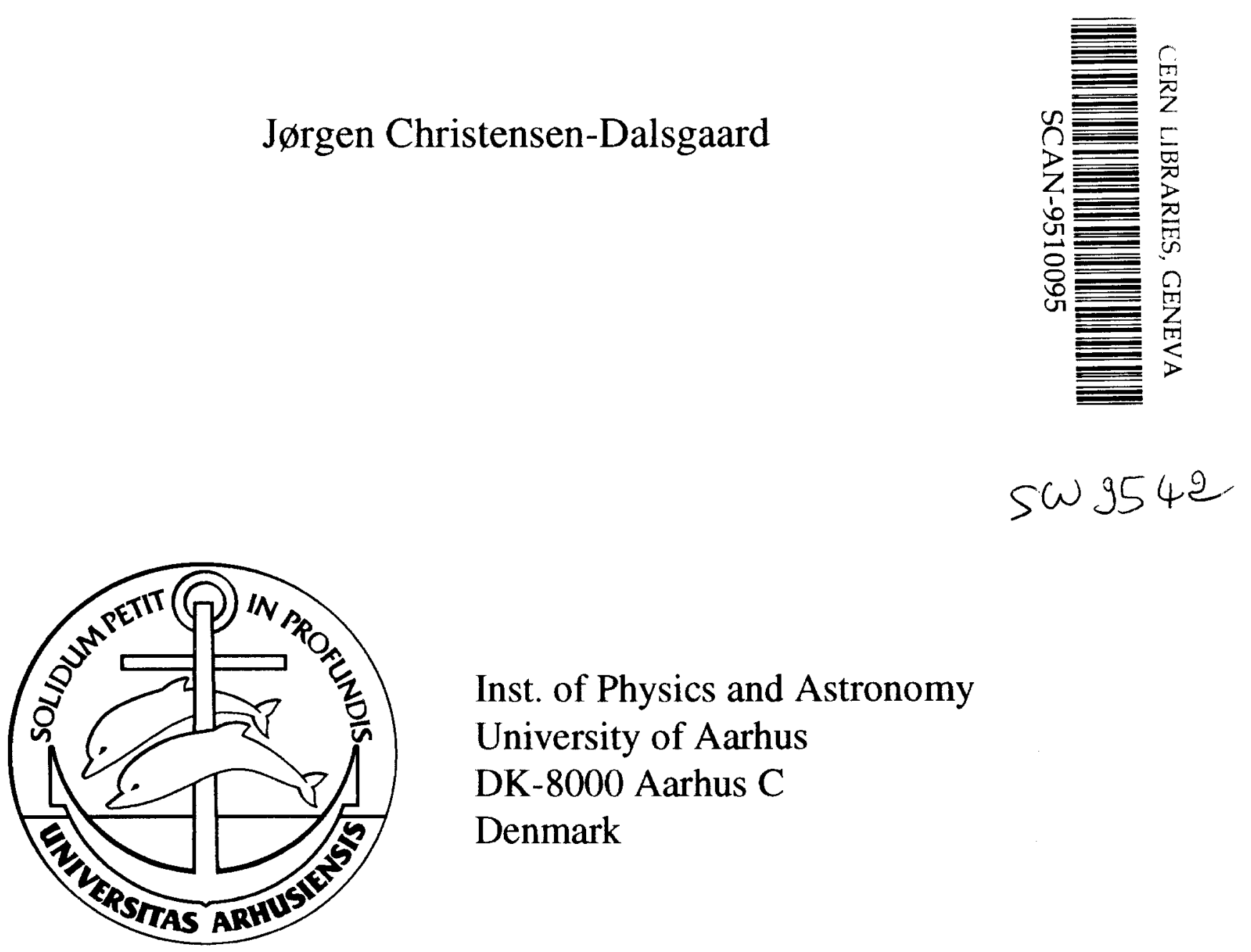

To appear in Proc. VI IAC Winter School "The Structure of the Sun", ed. T. Roca Cortes, Cambridge University Press 


\title{
Testing a solar model: the forward problem
}

\author{
Jorgen Christensen-Dalsgaard
}

Teoretisk Astrofysik Center, Danmarks Grundforskningsfond, and Institut for Fysik og Astronomi

Aarhus Universitet

Dh 8000 Aarhus C, Denmark 


\section{Contents}

Testing a solar model: the forward problem

by Jurgen ('hristensen-Dalsgaard

1 INTRODUCTION 3

1.1 A little history . . . . . . . . . . . . . . . . . 3

1.2 Definition of the forward problem ........... 4

2 PHYSICS OF SOLAR MODELS 5

2.1 Introduction . . . . . . . . . . . . . . . 5

2.2 Microphysics. . . . . . . . . . . . . . . . . . . . . . . . . 6

2.2 .1 Equation of state . . . . . . . . . . . . 6

2.2 .2 Opacity . . . . . . . . . . . . . . . 8

2.2 .3 Nuclear reactions . . . . . . . . . . . . . . 8

2.2.4 Microscopic diffusion and gravitational settling . . . . 9

2.3 Macrophysics . . . . . . . . . . . . . . . 10

2.3 .1 Outer convection zone . . . . . . . . . . 10

2.3 .2 Mixing in the solar interior? . . . . . . . . . 11

2.4 Structure of the solar convection zone . . . . . . . . 12

2.5) Overview of solar structure . . . . . . . . . . . 13

3 PROPERTIES OF ADIABATIC OSCILLATIONS 15

3.1 Introduction . . . . . . . . . . . . . . 15

3.2 What do the oscillation frequencies depend on? . . . . . 16

3.3 Simple p-mode asymptotics . . . . . . . . . . . . 18

3.3.1 Simple derivation of Duvall law . . . . . . . . 18

3.3.2 Effects of changing the dispersion relation . . . . . . . 19

3.3.3 Asymptotic effects of changes in structure . . . . . . 20

3.3.4 Effect of perturbation in gravitational potential . . . . 21

3.3.5 Refinements of asymptotic theory . . . . . . . . . . 22

3.3.6 Asymptotics of low-degree modes . . . . . . . . . . . 23

3.4 Perturbation analysis of effects of model changes . . . . . . . . 24

3.4.1 Reformulation of the oscillation equations . . . . . . . 25

3.4.2 Effects of changes in solar structure . . . . . . . . . 26

3.4 .3 Effects of sharply localized feature . . . . . . . 28

3.4 .4 Effects of near-surface modifications . . . . . . . . . 31

4 APPLICATION TO MODELS AND OBSERVED FREQUENCIES 
1.1 Effects of changes in the superadiabatic region of the convection

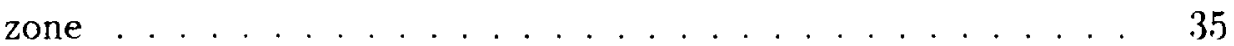

4.2 Opacity increase near the base of the convection zone . . . . 36

4.3 Diffusion and settling of helium . . . . . . . . . . 39

4.4 Opacity decrease in the core . . . . . . . . . . . . . . 44

4.5 The base of the convection zone . . . . . . . . . . . 47

4.6 The equation of state .................. 50

4.6.1 Comparison of EFF and CEFF formulations . . . . . 51

4.6.2 Comparison of CEFF and MHD formulations . . . . 55

5 ANALYSIS OF OBSERVED FREQUENCIES $\mathbf{5 5}$

5.1 Asymptotic analysis of observed frequencies . . . . . . . . 56

5.2 Test of the equation of state .................. 61

5.3 Matching the near-surface effects . . . . . . . . . 67

6 TOWARDS THE STARS? $\quad 69$

$6.1 \eta$ Bootis . . . . . . . . . . . . . . . . . 71

6.2 Concluding remarks . . . . . . . . . . . . . . . 79

$\begin{array}{llr}7 & \text { REFERENCES } & \mathbf{8 1}\end{array}$ 


\section{INTRODUCTION}

The present chapter addresses the forward problem, i.e., the relation between lhe structure of a solar model and the corresponding frequencies. As important, howerer, is the extent to which the frecuencies reflect the physics and other assumptions underlying the model calculation. Thus in Section 2 I consider some aspects of solar morlel computation. In addition, the understanding of the diagnostir potential of the frecpurncies requires information about the properties of the oscillations, beyond what was supplied in the chapter by Leibacher. This is provided in Section 3. Section 4 investigates the relation between the properties of solar structure and the oscillations by considering several examples of modifications to the solat models and their effects on the frequencies, while Section 5 consicters further analyses of the observed frequencies. Finally, the prospects of extending this ype of work to other stars are addressed in Section 6.

The present clapter assumes as background the chapter by Leibacher in this volume. Other general presentations of the properties of solar and stellat oscillations have been given by for example. Thuo et al. (1989), (iough \& 'Fommer (19991). (iongh (1993) and ('hristensen-Daliggard (1994).

\section{$1.1 \quad$ A little history}

The realization that observed frequencies of solar oscillation might provide information about the solar interior goes back at least two decades. Observations of fluctuations in the solar limb intensity (Hill \&. Stebbins 1975; Hill, Stebbins (A. Brown 1976), and the (laimed detection of a Doppler velocity oscillation with a period close to 160 minutes (Brookes, Isaak \& vau der Raay 1976; Severny, Kotov \& Tsap 1976) provided early indications that global solar oscillations might be detectable and led to the first comparisons of the reported frequencies with those of solar models (e.g. Scuflaire et al. 1975; Christensen-Dalsgaard \& (iough 1976; Iben \& Mahaffy 1976; Rouse 1977). Although the reality of these carly claims is questionable, they undoubtedly provided an important starting point for this type of work.

At about the same time the first detailed observations of the five-minute oscillations of high degree (Deubner 1975; Rhodes. Ulrich \& Simon 1976) confirmed their nature as trapped acoustic modes of oscillation in the outermost parts of the Sun, previously inferred by Ulrich (1970) and Leibacher \& Stein (1971). More detailed computations of frecuencies for solar envelope models yielded results in overall agreement with the observations and suggested that the observed modes were overstable (e.g. Ando \&: Osaki 1975, 1977; it should 
be noted that later stability analyses, taking into account the interaction with convection, indicate that the modes are stable; sce Balmforth 1992a). Further comparisons of the observed and computed frequencies inclicated that the solar convection zone was deeper than previously assumed (Gough 1977a; Ulrich \&: Rhodes 1977) and showed that the frequencies were sensitive to details of the equation of state of matter in the Sun (e.g. Berthonieu et al. 1980; Lubow, Rhodes \& Ulrich 1980).

The first definite detection of modes extending through most of the Sun resulted from whole-disk Doppler measurements which clearly showed oscillations in the five-minute region (Fossat \& Ricort 1975; Claverie et al. 1979); particularly important was the identification in the latter data of an approximately uniformly spaced set of peaks in the power spectrum, corresponding to the asymptotically predicted behaviour of the frequencies of low-degree acoustic modes. A much more detailed spectrum, resolving individual modes, was obtained in almost continuous observations over several days from the geographical South Pole (Grec, Fossat \& Pomerantz 1980).

The connection between these low-degree modes and the high-degree oscillations mentioned above was established by observations by Harvey \& Duvall (1984). This enabled an unambiguous identification of the radial orders of the low-degree modes and provided extensive data on the structure of the solar interior. Further observations using a variety of techniques have since then dramatically increased the number of identified modes and the accuracy with which the frequencies have been determined, providing the current basis for helioseismic investigations.

\subsection{Definition of the forward problem}

Given a set of observed frequencies, probably the most :mmediate and obvious method of analysis is to compare them with frequencies computed for a solar model. This essentially defines the forward problem, as a test of a solar model. As such, the oscillation frequencies have several major advantages over other measurements that might relate to the structure of the solar interior: they can be determined observationally with great accuracy; different modes probe very different aspects of the structure; and, given a solar model, the frequencies can be computed with substantial precision. As discussed in more detail below the last statement must be qualified: aspects of the superficial region of the Sun introduce uncertainties in the computed frequencies which must be kept in mind when carrying out the comparison with the observations. Nonetheless, the frequencies remain very powerful diagnostics of the solar interior. 
In some sense the solar model in itself is uninteresting: what requires testing are the assumptions and physical properties that underly the calculation of the model, thus improving the basis for general stellar-evolution calculations. Furhliemore. the accuracy and extent of the solar data allow the properties of matter in the Sun to be probed in considerable detail, thus providing information that is totally inaccessible in laboratory studies. In this sense, therefore, the forward problem link the physics to the observed frequencies. This point of view will be employed extensively in the following.

Facel with the inevitable discrepancies between the computed and observed frecpuencies, what does one do? The obvious goal is to correct the model, or more fundanentally the physics, in such a way as to reduce the discrepancies. (One approach is to compute several models and frequencies, adopting that model which best fits the data. At the opposite extreme, techniques for inverse analysis (sce the chapter by (iongh) offer systematic ways of determining the corrections. In between is a grey area of least-squares fits to the data, varying small sets of suitably chosen paraneters. Such procedures were employed extensively in the allalysis of the carly, limited helioseismic data (c.g. Christensen-Dalsgaard \& (iough 1980; (iabriel. Sicullaire \& Noels 1982). Although the current wealth of lata nakes inverse lechnigues attractive, parancter fitting still has an important role 10 play for more specialized applications, where the data can be combined in such a way as to inolate specific aspects of the solar interior. An example of this will be considered in Section 5.2 .

\section{PHYSICS OF SOLAR MODELS}

\subsection{Introduction}

The computed solar models, and hence their frequencies, depend on assumptions about the physical properties of matter in stars, in particular the equation of state, the opacity and the rates of nuclear reactions; these aspects of the calculation might be called the microphysics. Furthermore, the computations involve a number of simplifying assumptions, often covering much complex physics which might be called the macrophysics:

- The treatment of convection is approximated by mixing-length theory which provides a parametrization of the structure of the uppermost part of the convection zone in terms of the mixing-length parameter $\alpha_{c}$.

- The dynamical effects of convection (the so-called turbulent pressure) are ignored. 
- It is assumed that there is no mixing outside convectively unstable regions.

- Effects of magnetic fields are ignored.

Similarly, the calculations of oscillation frequencies are often done in the adiabatic approximation. Even when nonadiabatic effects are taken into account, their treatment is uncertain, since there is no definite theory for the perturbation in the convective flux, induced by the oscillations. Also, the perturbations in the turbulent pressure are usually neglected.

The goals of the analysis of observed frequencies are evidently to test both the microphysics and the simplifying assumptions. This is complicated by the fact that a given region of the model in general is affected by several aspects of the microphysics, $e . g$. both the opacity and the equation of state; under these circumstances it may evidently be difficult or impossible to isolate the cause of discrepancies between observations and models.

The computation of solar models requires the specification of a number of parameters. The age of the Sun can be estimated from ages determined for meteorites (e.g. Guenther 1989; Appendix by ( $;$. Wasserburg in the paper by Bahcall \& Pinsonneault 1995). The present ratio $Z / X$ between the abundances of heavy elements and hydrogen on the solar surface is approximately known from spectroscopy (e.g. Anders \& Grevesse 1989; Grevesse \& Noels 1993). Also, the computed models must match the photospheric radius and surface luminosity of the present Sun. This is achieved by adjusting the initial abundance $Y_{0}$ of helium and a parameter characterizing convective energy transport. The latter parameter (in mixing-length theory taken to be the ratio $\alpha_{c}$ between the mixing length and the pressure scale height) serves to fix the value $s$ of the specific entropy in the bulk of the convection zone, where the temperature stratification is essentially adiabatic and where $s$ is therefore nearly constant.

In the following I provide a brief overview of the some aspects of the physics of particular relevance to the analysis of the oscillation frequencies.

\subsection{Microphysics}

\subsubsection{Equation of state}

As mentioned in Section 1.1 the potential for using the observed frequencies to test the equation of state was recognized quite early. A detailed analysis of the treatment of the thermodynamical properties of solar matter and its effect on the frequencies was given by Christensen-Dalsgaard \& Däppen (1992). 
The theoretical description of the solar plasma is complicated by the interactions between its constituents, which strongly affect. e.g. the degree of partial ionization in the onter parts of the Sun. A specific problem is to ensure that matter becomes fully ionized in the deep solar interior; this is normally achieved by including some formulation for the so-called "pressure ionization", taking effert at high density. We are still far removed from a definitive trealment of these processes. However, an essential feature of any treatment is that it be thermodynamically consistent. If this is not the case, the results obtained depend on the details of how. for example. the oscillation equations are formulated, potentially lading to umpredictable results.

Here 1 consider the following equations of state:

- The Figgleton, Faulkner \& Flannery (1973) formulation (in the following $\mathrm{EFF}$. This uses the hasic Saha equation, assuming all atoms or ions to be in the ground state, and includes a simple but thermodynamically consislent treatment of pressure ionization.

- The ('EFF formulation. This corresponds to EFF, but with the addition of ('oulomb effects in the Debye-Hückel approximation. It should be noted that these effects result in corrections to pressure and internal energy of the gas, as well as to the chemical potentials and hence ionization balance. The effects on ionization, which dominate in the outer parts of the model, liave sometimes been ignored, leading to thermodynamic inconsistencies.

- The so-called MHI) formulation (Hummer \& Mihalas 1988; Mihalas, Däppen \& Hummer 1988; Mihalas et al. 1990). This provides a detailed treatment of the interactions within the gas, involving a probabilistic decr.ription of the level populations.

These formulations are all based on what has been called the chemical picture (e.g. Däppen 1992). where the constituents of the gas are regarded as atoms, ions and electrons. However, equally detailed descriptions exist in the physical picture, where the properties of the gas are described directly in terms of the interactions between fundamental particles, handled through many-body activity expansion. For practical applications the most important example is the Livermore equation of state (e.g. Rogers. Swenson \& Iglesias 1995), which forms the basis for the OPAL opacities discussed in the following section. 


\subsubsection{Opacity}

Incertainties in the opacity have substantial effects on the solar models and frequencies. Early inversions for the sound speed (Christensen-Dalsgaard et al. 1985) indicated that the the opacity should be increased in the solar interior, relative to the then used tables. Such increases have in fact been found in recent opacity calculations, substantially reducing the discrepancies in the structure of the radiative interior between the models and the Sun. Nonetheless, it is likely that some of the remaining difference may still be caused by errors, at a level of a few per cent, in the opacities. A particular uncertainty concerns the opacity in the solar atmosphere: much of the difference between the observed and computed frequencies can be eliminated through a substantial increase in the low-temperature opacity ( $c f$. Section 5.3), although the reality of such large opacities is doubtful.

Of particular importance in the recent revisions of the opacity calculations lias been the inclusion of large numbers of spectral lines, which have been found to dominate the opacity in extensive regions of the density-temperature plane. The result has been increases in opacity of up to factors of $2-3$, although the changes under conditions relevant to the Sun have been somewhat smaller. Two independent calculations, using rather different techniques, have been carried out: by the Opacity Project (OP; e.g. Seaton et al. 1994); and by the Livermore group (e.g. Iglesias et al. 1992) resulting in the so-called OPAL table. The results of these two sets of calculations agree to within a few per cent.

The models discussed in this chapter were computed with the OPAL tables; the low-temperature opacities were obtained from Kurucz (1991). To illustrate the sensitivity to the opacity I also consider several cases of modifying artificially the opacity in restricted temperature ranges.

\subsubsection{Nuclear reactions}

The nuclear reactions responsible for the Sun's energy output are discussed in the chapter by Bahcall. The details of the reaction networks are of crucial importance to the computed neutrino fluxes. In contrast, the oscillation frequencies are relatively insensitive to the reaction parameters. This is to some extent caused by the calibration of the initial composition to obtain the correct luminosity for the model of the present Sun: a change in the reaction parameters is compensated by a change in the composition, leaving the structure of the model largely unchanged. Dziembowski et al. (1994) found that a 3 per cent change in the basic $p-p$ reaction rate caused changes in sound speed and density which are barely detectable with the present helioseismic accuracy. They also considered 
an increase in the ${ }^{3} \mathrm{He}+{ }^{3} \mathrm{He}$ rate of a sufficient magnitude to bring the computed capture rate in the ${ }^{37} \mathrm{Cl}$ radiochemical neutrino experiment into agreement with the measurements; interestingly, this resulted in changes in sound speed and density which might well be detectable in the oscillation frequencies, at least if other uncertainties in the structure of the model could be eliminated.

Here I largely employ nuclear parameters from Parker (1986).

\subsubsection{Microscopic diffusion and gravitational settling}

Microscopic diffusion and gravitational settling are not normally considered part of a "standard model calculation". Indeed, crude estimates (e.g. Eddington 1926) suggest that the average diffusive time scale far exceeds the typical lifetime of stars. However, more careful calculations show that the effect is significant in solar evolution (c.g. Noerdlinger 1977; Wambsganss 1988), particularly in view of the high precision with which solar models can be tested.

An initial study of the effects of diffusion and settling on solar oscillations was carried out by Demarque \& Guenther (1988). Cox, Guzik \& Kidman (1989) considered detailed effects on solar models and oscillation frequencies. The dominant effect is the settling of helium out of the convection zone. Solar models with diffusion were also computed by Proffitt \& Michaud (1991), while ChristensenDalsgaard, Proffitt \& Thompson (1993) showed that helium settling, causing a steep gradient in the helium abundance at the base of the convection zone, very substantially improved the agreement between the sound speed of the model and the solar sound speed as inferred from helioseismic inversion. In addition, there was a significant effect of the accumulation of helium in the core. These results are discussed in Sections 4.3 and 5.1.

Settling similarly causes the heavy elements to sink, slightly depleting the convection zone and the outer parts of the radiative interior and enriching the core. This mainly affects the structure through a modification of the opacity in the radiative interior. Also, the current photospheric value of $Z / X$ is smaller than the initial value. Proffitt (1994) found that when this effect was taken into account, the resulting sound-speed profile below the convection zone, but excluding the core, was rather similar to the result obtained with helium settling and diffusion alone. It should be noted, however, that different heavy elements diffuse at different rates. With diffusion and settling the relative mixture of the heavy elements therefore varies with position. This causes very substantial complications, which so far have not been dealt with consistently, in the interpolation in the opacity tables. 


\subsection{Macrophysics}

\subsubsection{Outer convection zone}

There seems little doubt that most of the solar convection zone is very nearly adiabatically stratified: here a minute superadiabaticity is sufficient to drive the convective motion required for the energy transport. The calibration of solar models to the correct radius essentially fixes the structure of this region, and hence the depth of the convection zone (Gough \& Weiss 1976). In the uppermost part of the convection zone, however, the density is so low that energy transport requires a substantial superadiabatic gradient. Only here do different treatments of convection result in significant differences in solar structure.

In general, prescriptions for convection include free parameters, such as the mixing-length parameter $\alpha_{\mathrm{c}}$ in mixing-length theory. One such parameter is required for the calibration, controlling the change in specific entropy $s$ from the bottom of the atmosphere (where $s$ is essentially determined by atmospheric structure, as fixed by theoretical or semi-empirical atmospheric models) to the nearly adiabatic interior of the convection zone. This calibration fixes the integral of the superadiabatic gradient $\nabla-\nabla_{\mathrm{ad}}$, where $\nabla=\mathrm{d} \ln T / \mathrm{d} \ln p$ and $\nabla_{\mathrm{ad}}$ is its adiabatic value. However, the detailed behaviour of $\nabla-\nabla_{\text {ad }}$ differs between different treatments of convection. Canuto \& Mazzitelli $(1991,1992)$ developed descriptions based on assuming a full turbulent spectrum, and using as mixing length the distance to the top of the convection zone; this formulation resulted in a substantially higher and sharper $\nabla-\nabla_{\text {ad }}$ in the superadiabatic region than for traditional mixing-length models. Lydon et al. (1992) based their treatment on correlations obtained from hydrodynamical simulations of turbulent convection, finding $\nabla-\nabla_{\text {ad }}$ similar to, although slightly lower and broader than, the results of mixing-length theory. In this treatment, as well as the in the work of Canuto \& Mazzitelli, adjustable free parameters were not explicitly used. Monteiro, Christensen-Dalsgaard \& Thompson (1995ab) made an extensive survey of various types of convection formulations and their effects on the computed oscillation frequencies.

These convection treatments are all local, in that they assume that the convective flux at a given point is determined by the conditions, including the superadiabatic gradient, at that point. In reality convective eddies must sample a range of positions within the Sun, and the energy transport is determined by eddies originating over a range of levels. Procedures for taking such effects into account were developed by Spiegel (1963) and Gough (1976), although the application to solar modelling has been slow. Exceptions are the work by Xiong $\&$ Chen (1992) and by Balmforth (1992) who in addition considered effects of a time-dependent formulation of non-local mixing-length theory on solar oscilla- 
tions.

In the strongly superadiabatic region the convective velocities reach a substantial fraction of the sound speed. As a result, the convective momentum transport makes a significant contribution to hydrostatic balance in the average model. Nonctheless, this so-called turbulent pressure is usually ignored in computations of solar morels. Indeed, a consistent treatment of turbulent pressure in local mixing-length models is plagued by mathematical difficulties at the boundaries of the convectively unstable region (Gough 1977b). These problems are avoided in non-local treatments where there are no such sharp boundaries.

The near-surface part of the convection zone has also been modelled through detailed hydrodynamical simulations (c.g. Stcin \& Nordlund 1989). These show a strong asymmetry of the flow: rapid radiative cooling at the surface causes strong narrow cold downdrafts which persist throughout the computational domain. with slow upwelling in between. Fron such simulations mean models can be constructed through horizontal and temporal averaging. These models confirm that the stratification becomes nearly adiabatic at depth, with an adiabat which is relatively close to that obtained in calibrated mixing-length models. Furtherinore, the models clearly demonstrate the importance of turbulent pressure.

('onditions at the base of the convection zone remain uncertain. It seems inevitable that motion extends beyond the unstable region. Simple models (Shaviv \& Salpeter 1973; Schmitt, Rosner \& Bohn 1984; Pidatella \& Stix 1986; Zahn $1991)$ predict a nearly adiabatic extension of the convection zone with relatively vigorous motion, followed by an abrupt transition to the radiative gradient. As discussed in more detail in Section 4.5 the resulting near-discontinuity in the sound-speed gradient might have observable effects on the oscillation frequencies.

\subsubsection{Mixing in the solar interior?}

A potentially serious uncertainty in solar modelling concerns mixing caused by material motion in the solar interior. It is plausible that penetration beyond the convection zone induces weaker motion, possibly in the form of internal gravity waves, in the radiative interior. Such motion could have substantial effects on the composition profile resulting from microscopic diffusion and gravitational settling. Indeed, the reduction of the solar photospheric lithium abundance by roughly a factor 100 relative to the solar-system abundance indicates that mixing well beneath the convection zone must have occurred at some phase of solar evolution (e.g. Christensen-Dalsgaard, Gough \& Thompson 1992); lithium 
depletion in the pre-main-sequence phase may also have been an important factor (e.g. Ahrens, Stix \& Thorn 1992; Swenson et al. 1994). Schatzmann et al. (1981) considered the effects on solar evolution of substantial turbulent diffusion, finding that this might lead to a significant reduction in the predicted neutrino flux; the physical basis for the assumed diffusion coefficient was somewhat questionable, however.

The solar spin-down from a normally assumed initial rapid rotation is likely to have caused regions of strong shear in the solar radiative interior which could have led to instabilities and hence mixing. In an ambitious effort, Pinsonneault et al. (1989) have computed models of such effects, with approximate expressions for the transport resulting from the instabilities. The results showed substantial effects on the photospheric lithium abundance, reproducing the observed depletion, while the effect on the central hydrogen abundance was small. Detailed analysis of models including rotational instabilities indicated no significant changes in the oscillation frequencies (Chaboyer et al. 1995). It should be noted, however, that the computed rotation profiles seem to be inconsistent with helioseismic determinations of solar internal rotation (e.g. Duvall et al. 1984; Tomczyk, Schou \& Thompson 1995). Zahn (1992) developed a procedure to evaluate mixing due to turbulence induced by meridional circulation. Detailed results for the Sun have apparently so far not been obtained, however.

\subsection{Structure of the solar convection zone}

The fact that the bulk of the convection zone is nearly adiabatically stratified considerably simplifies the analysis of solar oscillation data. Here to a very good approximation pressure $p$ and density $\rho$ are related by

$$
\frac{\mathrm{d} \ln \rho}{\mathrm{d} \ln p} \simeq \frac{1}{\Gamma_{1}}
$$

where $\Gamma_{1}=(\partial \ln p / \partial \ln \rho)_{\text {ad }}$. As a result, the structure of the convection zone is independent of opacity: it is determined by the value of the specific entropy $s$, by the composition (uniform, because of the very efficient mixing) and the equation of state. Consequently, modes of oscillation that are trapped in the convection zone are ideally suited to test the properties of the equation of state and to determine the solar composition (see also Christensen-Dalsgaard \& Däppen 1992).

From equation (1) we may easily obtain an approximate expression for the adiabatic sound speed $c$. Introducing $u=p / \rho$ we have from the equation for 
hydrostatic support that

$$
\frac{\mathrm{d} u}{\mathrm{~d} r}=\frac{1}{\rho} \frac{\mathrm{d} p)}{\mathrm{d} r}\left(1-\frac{\mathrm{d} \ln \rho}{\mathrm{d} \ln p}\right) \simeq-\frac{\operatorname{Cim}}{r^{2}}\left(1-\frac{1}{\Gamma_{1}}\right),
$$

where $(i$ is the gravitational constant and $m$ is the mass interior to radius $r$. In equation (2) we may take $m \simeq M$, the total mass of the Sun, since the convection zone contains only about 2 per cent of the solar mass. Outside the dominant ionization zones of hydrogen and helium, which are confined to the outer $2-3$ per cent of the radius, we can furthermore assume $\Gamma_{1}$ to be constant. Within this rather crude approximation we can therefore integrate equation (2), to obtain

$$
u \simeq \operatorname{CiM}\left(1-\frac{1}{\Gamma_{1}}\right)\left(\frac{1}{r}-\frac{1}{R^{*}}\right)
$$

or

$$
c^{2}=\Gamma_{1} u \simeq G M\left(\Gamma_{1}-1\right)\left(\frac{1}{r}-\frac{1}{R^{*}}\right) ;
$$

here $R^{*}$, which serves as a constant of integration, is approximately equal to the photospheric rartius $R$ of the star. Thus in the deeper parts of the convection zone $c(r)$ is essentially determined by the total mass and radius of the Sun, as well as by (a suitable average of) $\Gamma_{1}$. It should also be noted that equation (2) relates the derivative of $u$, which can be determined from the observed oscillation frequencies, to the thermodynamic properties of the gas as described by $\Gamma_{1}$. This relation may therefore be used to determine the helium abundance of the convection zone (Däppen \&: Gough 1984, 1986) or to test the equation of state (Dziembowski, Pamyatnykh \& Sienkiewicz 1992).

\subsection{Overview of solar structure}

Figure 1 provides a summary of the physics and uncertainties of solar internal structure. In the radiative interior the situation is complicated by the simultaneous dependence on the equation of state, opacity, energy generation rate and composition profile. On the other hand, since matter is essentially fully ionized in this region the uncertainties introduced by the equation of state are substantially reduced. Also, I indicated in Section 2.2.3 that the details of nuclear reactions have modest effects on the oscillation frequencies. Thus in this part of the Sun we expect that the oscillations will be used predominantly to measure aspects of the opacity and the composition profile. As argued in Section 2.4 the convection zone, on the other hand, depends on composition, specific entropy and the equation of state. Furthermore, the composition can be assumed to be independent of position in the convection zone; this offers some hope that uncertainties in the composition and the equation of state can be partially separated 


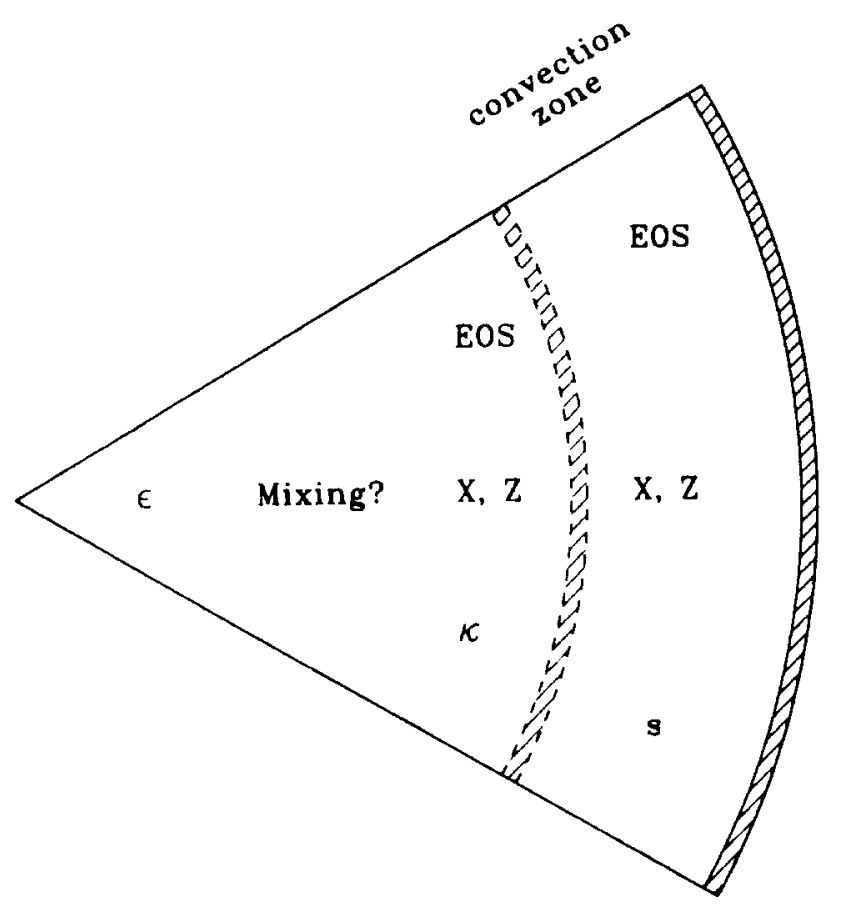

Figure 1: Schematic representation of solar structure. The thin hashed area near the surface indicates the region where the physics is uncertain, because of effects of convection, nonadiabaticity, etc. At the base of the convection zone, convective overshoot and diffusion introduce additional uncertainty. The structure of the adiabatic part of the convection zone is determined by the equation of state (EOS), and the constant values of specific entropy s, and composition (given by the abundances $X$ and $Z$ of hydrogen and heavy elements). Beneath the convection zone the structure also depends on opacity $\kappa$ and the energy generation rate $\epsilon$.

(Gough 1984a). It is obvious, however, that one cannot exclude errors in the equation of state which essentially mimic a change in composition.

Much of the uncertainty in the physics is concentrated very near the surface. This is true of the dynamical effects of convection, since convective velocities are likely to be very small elsewhere, and of the details of convective energy transport; furthermore, unless the interior field is much stronger than the observed photospheric field, effects of magnetic fields on the average solar structure are similarly concentrated. Also, as discussed by Leibacher (this volume) potential errors in the treatment of the oscillations, associated with nonadiabatic effects and fluctuations in the turbulent pressure, are concentrated in this region; in contrast, the oscillations are expected to be adiabatic to very high precision in the solar interior. These near-surface uncertainties have a substantial effect on 
the oscillation frequencies which may influence the analysis of the observations. Fortunately, as argued in Section 3.4 .4 it is to a large extent possible to separate the effects of the superficial errors, hence isolating those aspects of the frequencies which provide reliable information about the solar interior.

\section{PROPERTIES OF ADIABATIC OSCILLA- TIONS}

\subsection{Introduction}

The basic properties of solar oscillations are described in the chapter by leibacher. However. for completeness the present chapter contains certain basic definitions already given there. Also, in the following analysis of the sensitivity of the oscillation frepuencies to aspects of structure we shall need additional insight. Much of the material presented here has been adapted from ChristensenDalsgaard (1994).

Although claims lor detection of g modes have been made, the existing identified observations of solar oscillation are confined to $p$ and $f$ modes. Thus I shall restrict nyself to these modes. Furthermore, I generally assume the oscillations to be adiabatic. It was noted by Leibacher that nonadiabatic effects on the frequencies are important very near the surface; however, the physical nature of these effects is still badly understood, and so they must at present be regarded as a source of uncertainty in the analysis of the observed frequencies.

As usual, the modes are described in terms of spherical harmonics $Y_{l}^{m}$; the degree $l$ is related to the the length $k_{\mathrm{h}}$ of the horizontal component of the wavenumber, at distance $r$ from the centre, by

$$
k_{\mathrm{h}}=\frac{L}{r} . \quad \text { with } \quad L^{2}=l(l+1)
$$

We neglect rotation and other departures from spherical symmetry. Then the angular frequencies $\omega_{n l}$ depend only on the degree and the radial order $n$ of the modes. In addition to $\omega_{n i}$, we shall often consider the cyclic frequencies $\nu_{n l}=\omega_{n l} / 2 \pi$.

The frequencies of a solar model are complicated functions of the structure of the model. However, all available evidence indicates that our present solar models are fairly close to the actual solar structure. This motivates analyzing the observed frequencies in terms of departures from the frequencies of some reference 
model. If the differences between the Sun and the model are sufficiently small, there is an approximately linear relation between the model and the frequency differences (see also Section 3.4). Similarly, the effects of hanging some aspect of the model physics is conveniently described in terms of the corresponding model and frequency differences. Several examples of this are given in Section 4.

Computation of adiabatic frequencies for a given model is relatively straightforward. However, the results are not immediately easy to interpret. Insight into the relation between the frequencies and the structure of the model can be obtained from the asymptotic properties of the modes. Most of the observed frequencies correspond to acoustic modes of relatively high radial order or degree, for which the asymptotic description is fairly accurate. Although the asymptotic theory cannot replace precise numerical computation, it may still be used to obtain quantitative results. Examples of this are given in Section 5.1.

The f-mode frequencies depend only weakly on solar structure. To leading order, they satisfy

$$
\omega^{2} \simeq g_{\mathrm{s}} k_{\mathrm{h}},
$$

where $g_{\mathbf{s}}$ is the surface gravitational acceleration. To this accuracy the information content in the $f$-mode frequencies is clearly limited. The observed frequencies show departures from equation (6) which are substantially larger than the corresponding departures for computed frequencies for normal solar models; the origin of these departures is so far uncertain (e.g. Rosenthal \& Gough 1994; Rosenthal \& Christensen-Dalsgaard 1995; Rosenthal et al. 1995a). Thus in the following I shall predominantly consider p modes.

\subsection{What do the oscillation frequencies depend on?}

The adiabatic oscillation equations (see the chapter by Leibacher) obviously depend on the structure of the equilibrium model. A closer inspection reveals that the coefficients are determined solely by the set of equilibrium variables

$$
\rho, p, \Gamma_{1}, g
$$

as functions of $r$. However, the equilibrium model satisfies the stellar structure equations; in addition it may be assumed to have a given mass and radius, which at least in the case of the Sun are known with high precision. If $\rho(r)$ is given, the interior mass and hence $g(r)$ can be determined from simple integration; given $g$, the equation of hydrostatic support may be integrated from the surface to provide $p(r)$ (the surface pressure is known from empirical models of the solar atmosphere). Thus of the set (7) only the two functions $\rho(r)$ and $\Gamma_{1}(r)$ 
are independent, and the adiabatic oscillation frequencies are determined solely by these two functions. ('onversely, if no other constraints are imposed, the observed frequencies give direct independent information only about $\rho$ and $\Gamma_{1}$; from $\rho, p$ may then be determined from the constraint of hydrostatic support.

The equation of state relates $\Gamma_{1}$ to $p, \rho$ and the chemical composition. To a fair degree of approximation the chemical composition can be specified by a single parameter. such as the abundance $Y$ by mass of helium. In this approximation just three quantities should suffice to specify fully the thermodynamic state. (iven that $\rho, p$ and $\Gamma_{1}$ can be obtained from the oscillation observations, knowledge of the equation of state should enable the determination of any other thermodynamic variable, including $\zeta$. from these observed quantities. Outsicle the major ionization zones $\Gamma_{1}$ is very nearly constant and hence gives a poor determination of the thermodynamic state; however, it varies sufficiently in the helium ionization zones to allow a determination of $\zeta$, provided that the properties of the equation of state are known with sufficient accuracy. Indeed, procedures for such a determination of the helium abundance of the Sun have been proposed (e.g. Däppen \& Gough 1984. 1986; Vorontsov, Baturin \& Pamyatnyth 1991; Kosovichev of al. 1992). Alternatively, such analyses provide a test of the equation of state. if the helium abundance is otherwise constrained. As discussed in Section 5.2, an apparently quite efficient technique can be obtained on the basis of the asymptotic behaviour of the oscillations in the helium ionization zone.

The preceding discussion was made in terms of the pair $\left(\rho, \Gamma_{1}\right)$. However, any other independent pair of model variables, related directly to $\rho$ and $\Gamma_{1}$, may be used instead. Since most of the observed solar oscillations have essentially the nature of standing acoustic waves, their frequencies are largely determined by the behaviour of sound speed $c$; hence it is natural to use $c$ as one of the variables, combined with, e.g. $\rho$ or $\Gamma_{1}$. As discussed in Section 5.1 below, the observations are sufficiently rich that the observed frequencies may be inverted to obtain an estimate of the sound speed in most of the Sun. It follows from the equation of state, approximated by the ideal gas law, that this essentially provides a measure of $T / \mu, T$ being temperature and $\mu$ the mean molecular weight. However, it is important to note that measurements of adiabatic oscillation frequencies do not by themselves allow a determination of the temperature in a star. Only if the mean molecular weight can be otherwise constrained (e.g. by demanding that its variation in the stellar interior results from normal stellar evolution) is it possible to estimate the stellar interior temperature. This limitation is of obvious importance for the use of observed solar oscillation frequencies to throw a light on the apparent deficit of observed solar neutrinos (e.g. Christensen-Dalsgaard 1991). 


\subsection{Simple p-mode asymptotics}

The asymptotic properties of solar oscillations were discussed by Leibacher (this volume). Here I repeat and extend a few simple aspects of the asymptotic description, for use later in the present chapter.

\subsubsection{Simple derivation of Duvall law}

A relation central to the understanding of p-mode behaviour is the Duvall law. This can be derived very simply from the dispersion relation for plane sound waves:

$$
\omega^{2}=c^{2}|\mathbf{k}|^{2},
$$

where $\mathbf{k}$ is the wave number. I write $|\mathbf{k}|^{2}=k_{\tau}^{2}+k_{\mathrm{h}}^{2}$, where $k_{\mathrm{h}}$ is given by equation (5). Thus

$$
k_{r}^{2}=\frac{\omega^{2}}{c^{2}}-\frac{L^{2}}{r^{2}} .
$$

This equation describes the geometry of the ray along which the wave propagates; propagation, with real $k_{r}$, is confined to the region outside the turning point $r=r_{\mathrm{t}}$, where $r_{\mathrm{t}}$ satisfies

$$
\frac{c\left(r_{\mathrm{t}}\right)}{r_{\mathrm{t}}}=\frac{\omega}{L}
$$

A more careful analysis (briefly summarized in Section 3.3.5) shows that the waves have an outer turning point $R_{\mathrm{t}}$ located just below the photosphere. To obtain a standing wave (i.e., a mode of oscillation) we must require, roughly, an integral number of oscillations in the radial direction between the lower turning point and the photosphere, although taking into account the phase shifts at the extremes of the propagating region; thus

$$
\int_{r_{1}}^{R} k_{\tau} \mathrm{d} r=n(\pi+\alpha)
$$

where $\alpha$ takes care of the behaviour near the turning points. From equation (9) we therefore obtain

$$
\int_{r_{1}}^{R}\left(1-\frac{L^{2} c^{2}}{\omega^{2} r^{2}}\right)^{1 / 2} \frac{\mathrm{d} r}{c}=\frac{[n+\alpha(\omega)] \pi}{\omega},
$$

which is the Duvall law.

It might be noted that analysis of the asymptotics of low-degree modes indicates that in the asymptotic relations $L$ should be defined as

$$
L=l+1 / 2 \text {, }
$$


instead of the definition given in equation (5). (Note that the distinction between these two definitions is only significant for low-clegree modes.) In the following applications of the asymptotic theory equation (13) will be used.

\subsubsection{Effects of changing the dispersion relation}

To investigate the effects of changes to the model in the asymptotic approximation, we replace equation (8) by

$$
\omega^{2}=c^{2}|\mathbf{k}|^{2}+\delta f(r)
$$

where $\delta f$ is a small modification which, as indicated. is assumed to be given as a function of $r$. Instead of equation (9) we therefore obtain, to leading order in $\delta f$,

$$
\begin{aligned}
k_{r} & =\left(\frac{\omega^{2}}{c^{2}}-\frac{L^{2}}{r^{2}}-\frac{1}{c^{2}} \partial f\right)^{1 / 2} \\
& \simeq \frac{\omega}{c}\left[\left(1-\frac{L^{2} c^{2}}{\omega^{2} r^{2}}\right)^{1 / 2}-\frac{1}{2 \omega^{2}}\left(1-\frac{L^{2} c^{2}}{\omega^{2} r^{2}}\right)^{-1 / 2} \delta f\right] .
\end{aligned}
$$

By substituting equation (15) into the condition (11) for a standing wave we obtain

$$
\frac{(n+\alpha) \pi}{\omega} \simeq \int_{r_{1}}^{R}\left(1-\frac{L^{2} c^{2}}{\omega^{2} r^{2}}\right)^{1 / 2} \frac{\mathrm{d} r}{c}-\frac{1}{2 \omega^{2}} \int_{T_{\downarrow}}^{R}\left(1-\frac{L^{2} c^{2}}{\omega^{2} r^{2}}\right)^{-1 / 2} \delta f \frac{\mathrm{d} r}{c} .
$$

Here the last term shows the effect of the perturbation on the Duvall law.

We can now find the effect on the oscillation frequencies of the perturbation. We assume that the result is to change the frequency from $\omega$ to $\omega+\delta \omega$. Also it should be recalled that $\alpha=\alpha(\omega)$ in general depends on $\omega$. Finally, we note that the perturbation, in addition to $\delta f$, may involve a change $\delta \alpha$ to the phase function $\alpha$. Multiplying equation (16) by $\omega$ and linearizing in $\delta \omega$ and $\delta \alpha$ yields

$$
\begin{aligned}
\pi \frac{\mathrm{d} \alpha}{\mathrm{d} \omega} \delta \omega= & \delta \omega \int_{r_{1}}^{R}\left(1-\frac{L^{2} c^{2}}{\omega^{2} r^{2}}\right)^{1 / 2} \frac{\mathrm{d} r}{c}+\omega \int_{r_{\mathrm{t}}}^{R}\left(1-\frac{L^{2} c^{2}}{\omega^{2} r^{2}}\right)^{-1 / 2} \frac{L^{2} c^{2}}{\omega^{2} r^{2}} \frac{\delta \omega}{\omega} \frac{\mathrm{d} r}{c} \\
& -\frac{1}{2 \omega} \int_{r_{\mathrm{l}}}^{R}\left(1-\frac{L^{2} c^{2}}{\omega^{2} r^{2}}\right)^{-1 / 2} \delta f \frac{\mathrm{d} r}{c}-\pi \delta \alpha .
\end{aligned}
$$

From this we finally obtain

$$
S \frac{\delta \omega}{\omega} \simeq \frac{1}{2 \omega^{2}} \int_{r_{\mathfrak{l}}}^{R}\left(1-\frac{L^{2} c^{2}}{\omega^{2} r^{2}}\right)^{-1 / 2} \delta f \frac{\mathrm{d} r}{c}+\pi \frac{\delta \alpha}{\omega},
$$


where

$$
S=\int_{r_{\mathrm{t}}}^{R}\left(1-\frac{L^{2} c^{2}}{\omega^{2} r^{2}}\right)^{-1 / 2} \frac{\mathrm{d} r}{c}-\pi \frac{\mathrm{d} \alpha}{\mathrm{d} \omega} .
$$

'This is the desired general expression.

If the terms in $\mathrm{d} \alpha / \mathrm{d} \omega$ and $\delta \alpha$ are neglected, equation (18) has a very simple physical interpretation: the equation shows that the relative change in $\omega^{2}$ is just a weighted average of $\delta f / \omega^{2}$, with the weight function

$$
\mathcal{W}(r)=\frac{1}{c}\left(1-\frac{L^{2} c^{2}}{\omega^{2} r^{2}}\right)^{-1 / 2} .
$$

It is easily seen that $\mathcal{W}(r) \mathrm{d} r$ is just the sound travel time, corresponding to the radial distance $\mathrm{d} r$, along the ray describing the mode. Hence the weight in the average simply gives the time that the mode, regarded as a superposition of plane waves, spends in a given region of the star.

\subsubsection{Asymptotic effects of changes in structure}

To investigate the effects of modifications in solar structure, we consider a change in the sound speed from $c$ to $c+\delta c$; as a result, the dispersion relation for sound waves is changed to

$$
\omega^{2}=c^{2}|\mathbf{k}|^{2}+2 c \delta c|\mathbf{k}|^{2}=c^{2}|\mathbf{k}|^{2}+2 \omega^{2} \frac{\delta c}{c} .
$$

This is of the form given in equation (14), with $\delta f=2 \omega^{2} \delta c / c$. It follows from equation (18) that the frequency change is given by

$$
S \frac{\delta \omega}{\omega} \simeq \int_{r_{1}}^{R}\left(1-\frac{L^{2} c^{2}}{\omega^{2} r^{2}}\right)^{-1 / 2} \frac{\delta c \mathrm{~d} r}{c} \frac{\delta \alpha}{c}+\pi \frac{\delta}{\omega}
$$

Note that equation (22) is of the form

$$
S \frac{\delta \omega}{\omega} \simeq \mathcal{H}_{1}\left(\frac{\omega}{L}\right)+\mathcal{H}_{2}(\omega)
$$

where

$$
\mathcal{H}_{1}(w)=\int_{r_{1}}^{R}\left(1-\frac{c^{2}}{r^{2} w^{2}}\right)^{-1 / 2} \frac{\delta c}{c} \frac{d r}{c}
$$

and

$$
\mathcal{H}_{2}(\omega)=\frac{\pi}{\omega} \delta \alpha(\omega)
$$


Some properties of this equation were discussed by Christensen-Dalsgaard, Ciough \& Pérez Hernández (1988) and Christensen-Dalsgaard, Gough \& Thompson (1989). As pointed out in the latter reference. $\mathcal{H}_{1}(\omega / L)$ and $\mathcal{H}_{2}(\omega)$ can be obtained separately, to within a constant, by means of a double-spline fit of the expression (23) to p-mode freculuency differences. The dependence of $\mathcal{H}_{1}$ on $\omega / L$ is determined by the sound-speed difference throughout the star, whereas $\mathcal{H}_{2}(\omega)$ depends on differences in the upper lavers of the models.

\subsubsection{Effect of perturbation in gravitational potential}

The derivation of the Duvall law implicitly made the Cowling approximation, neglecting the perturbation $\Phi^{\prime}$ in the gravitational potential. It is very simple to estimate the effect on the frequencies of including $\Phi^{\prime}$. For plane acoustic waves, including the effect of self-gravity, the dispersion relation is

$$
\omega^{2}=c^{2}|\mathbf{k}|^{2}-4 \pi(i \rho
$$

(Jeans 1929). This is again of the form (14), with $\delta f=-4 \pi G \rho$. It follows from equation (16) that the modified Duvall law is

$$
\frac{\pi(n+\alpha)}{\omega} \simeq \int_{r_{1}}^{R}\left(1-\frac{L^{2} c^{2}}{\omega^{2} r^{2}}\right)^{1 / 2} \frac{\mathrm{d} r}{c}+\frac{2 \pi G}{\omega^{2}} \int_{r_{t}}^{R} \rho\left(1-\frac{L^{2} c^{2}}{\omega^{2} r^{2}}\right)^{-1 / 2} \frac{\mathrm{d} r}{c} .
$$

Note that, as $r_{\mathrm{t}}$ is a function of $\omega / L$. equation (27) may be written as

$$
\frac{\pi(n+\alpha)}{\omega}=F\left(\frac{\omega}{L}\right)+\frac{1}{\omega^{2}} F_{\Phi}\left(\frac{\omega}{L}\right)
$$

where the functions $F(w)$ and $F_{\Phi}(w)$ are defined by equation (27). An expression of this form, with additional correction terms, was obtained by Vorontsov (1991).

From equations (18) and (19) we obtain an approximate expression for the difference $\delta \omega^{(\Phi)}=\omega^{(F)}-\omega^{(C)}$ between the frequency $\omega^{(F)}$ obtained taking the perturbation in the gravitational potential into account, and the frequency $\omega^{(C)}$ obtained in the Cowling approximation. The result is

$$
\delta \omega^{(\Phi)} \simeq-\frac{1}{\omega} \frac{2 \pi G \int_{r_{1}}^{R} \rho\left(1-\frac{L^{2} c^{2}}{\omega^{2} r^{2}}\right)^{-1 / 2} \frac{\mathrm{d} r}{c}}{\int_{r_{1}}^{R}\left(1-\frac{L^{2} c^{2}}{\omega^{2} r^{2}}\right)^{-1 / 2} \frac{\mathrm{d} r}{c}} .
$$

Thus the frequency change induced by the gravitational potential perturbation depends on an average of the density structure of the equilibrium model, over the region where the mode is trapped. 


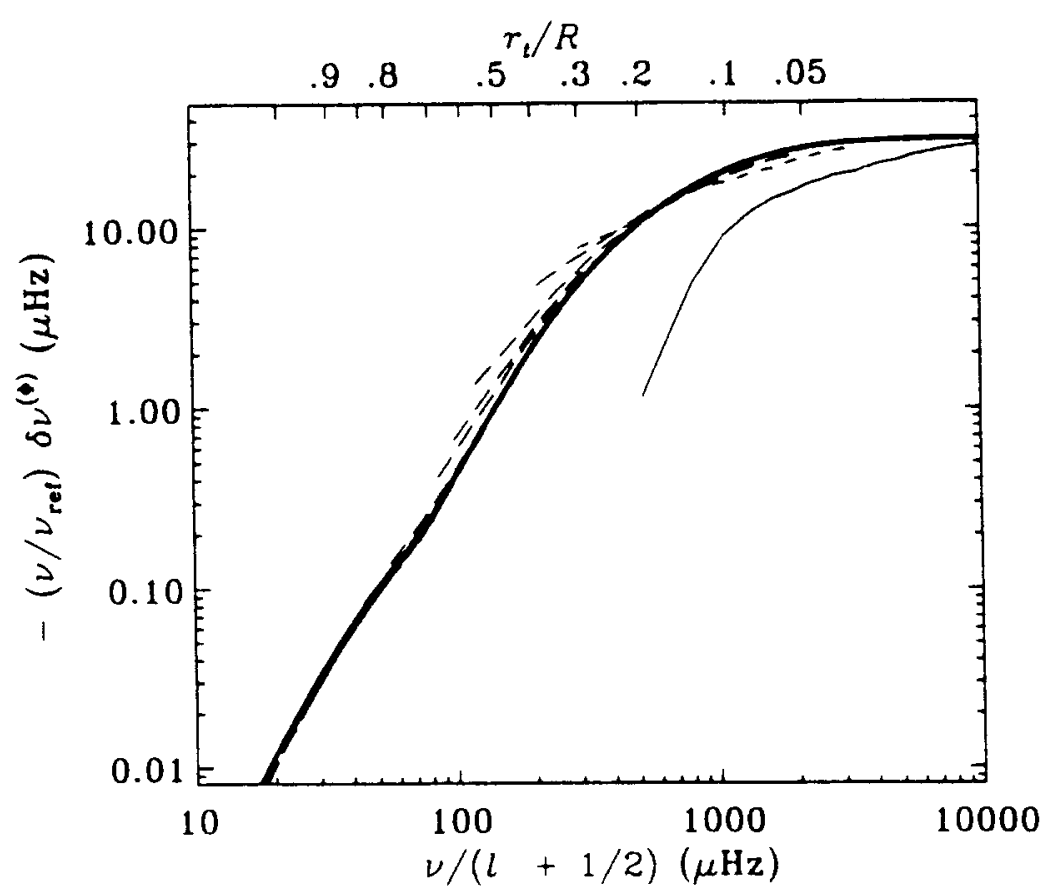

Figure 2: The thin lines show scaled frequency corrections $\delta \nu^{(\Phi)}$ resulting from the inclusion of the effect of the perturbation in the gravitational potential, for a normal solar model. Points corresponding to a given degree have been connected with a continuous line $(l=0)$, short dashes $(l=1)$ or long dashes $(l>1)$. In accordance with the asymptotic expression (29) $\delta \nu^{(\Phi)}$ has been scaled by $-\nu / \nu_{\text {ref }}$, using a reference frequency $\nu_{\mathrm{ref}}=3000 \mu \mathrm{Hz}$, and the results have been plotted against $\nu /(l+1 / 2)$ (lower abscissa) and $r_{\mathrm{l}} / R$ [cf. equation (10); upper abscissa]. The heavy continuous line corresponds to the asymptotic frequency difference $\delta \nu^{(\Phi)}=\delta \omega^{(\Phi)} / 2 \pi$ computed from equation (29).

It might be noticed that equation (29) directly relates the frequencies to the density structure of the model. However, the effect is evidently strongly diminished for modes of moderate or high degree, which do not penetrate into the high-density region near the core. This is illustrated in Figure 2 which shows the asymptotic and computed frequency corrections resulting from including the perturbation in the gravitational potential.

\subsubsection{Refinements of asymptotic theory}

The preceding asymptotic analysis was highly simplified, although sufficient for much of the following. For completeness, I here note some extensions.

It was shown by Gough ( $c f$. Deubner \& Gough 1984) that a more accurate 
asymptotic representation. valid both for pand $g$ modes, is

$$
\omega \cdot \int_{r_{1}}^{r_{2}}\left[1-\frac{\omega^{\prime 2}}{\omega^{\prime 2}}-\frac{L^{2} c^{2}}{r^{2} \omega^{\prime 2}}\left(1-\frac{N^{2}}{\omega^{2}}\right)\right]^{1 / 2} \frac{d r}{c} \simeq \pi(n-1 / 2),
$$

where $r_{1}$ and $r_{2}$ are adjacent zeros of the integrand. Here the characteristic acoustical cut-off frequency $u_{i}$ is defined by

$$
\omega_{i}^{2}=\frac{c^{2}}{4 H^{2}}\left(1-2 \frac{d H}{d r}\right) .
$$

where we have introduced the density scale height $H$ by

$$
H^{-1}=-\frac{\mathrm{d} \ln \rho}{\mathrm{d} r}
$$

also. $r$ is the buoyancy frequency. Near the surface, the term in $\omega_{c}$ dominates and defines the point $r=R_{1}$, with $\omega_{c}\left(R_{t}\right) \simeq \omega$. where the mode is reflected: this occurs essentially at the photosphere although for frequencies below about $2000 \mu \mathrm{Hz}$ the reflection point shifts deeper with decreasing frequency. Waves with frequencies exceeding the (nearly constant) value of $\omega_{c}$ in the atmosphere (corresponding to a cyclic frequency $\nu_{\text {ac }} \simeq 5300 \mu \mathrm{Hz}$ ) are essentially free to travel ont into the solar atmosphere, and hence do not form normal modes (see, however, the chapter by Brown).

One may obtain the original form of the Duvall law, equation (12), from equation (30) by neglecting the term in $N^{2}$ and expanding the bracket in the integral on the left-hand side to take out the dependence on $\omega_{\mathrm{c}}^{2}$ (e.g. ChristensenDalsgaard \& Pérez Hernández 1992); the contribution from $\omega_{\mathrm{c}}^{2}$ is essentially absorbed in $\alpha(\omega)$. A more careful analysis (Brodsky \& Vorontsov 1993; Gough $\&$ Vorontsov 1995) results in a further refinement of the Duvall law. The result may be written as

$$
F\left(\frac{\omega^{\prime}}{L}\right)+\frac{1}{\omega^{2}} F_{2}\left(\frac{\omega}{L}\right)=\pi \omega^{-1}\left[n+\alpha(\omega)+\left(\frac{L}{\omega}\right)^{2} \alpha_{2}(\omega)\right] .
$$

Here $F(w)$ is defined by equations (27) and (28), while $F_{2}(w)$ is analogous to $F_{\Phi}(w)$ introduced in those equations, but includes also a contribution from $N^{2}$ as well as other terms. The term in $\alpha_{2}(\omega)$ on the right-hand side arises because the waves propagate obliqueiy at the upper turning point. It becomes of substantial importance for modes whose degree exceeds a few hundred.

\subsubsection{Asymptotics of low-degree modes}

For low-degree modes one may show, by expanding the Duvall law, that

$$
\nu_{n l} \simeq\left(n+\frac{l}{2}+\frac{1}{4}+\alpha\right) \Delta \nu-\left(A L^{2}-\delta\right) \frac{\Delta \nu^{2}}{\nu_{n l}} .
$$


where

$$
\Delta \nu=\left[2 \int_{0}^{R} \frac{\mathrm{d} r}{c}\right]^{-1}
$$

is the inverse of twice the sound travel time between the centre and the photosphere, and

$$
A=\frac{1}{4 \pi^{2} \Delta \nu}\left[\frac{c(R)}{R}-\int_{0}^{R} \frac{\mathrm{d} c}{\mathrm{~d} r} \frac{\mathrm{d} r}{r}\right]
$$

(є.g. Tassoul 1980; Gough 1986). Neglecting the term in A, equation (34) predicts a uniform spacing $\Delta \nu$ in $n$ of the frequencies of low-degree modes. Also, modes with the same value of $n+l / 2$ are predicted to be almost degenerate,

$$
\nu_{n l} \simeq \nu_{n-1, l+2}
$$

As discussed by Leibacher (this volume) this frequency pattern has been observed for the solar five-minute modes of low degree and may be used in the search for stellar oscillations of solar type. The deviations from the simple relation (37) have considerable diagnostic potential. They may be expressed in terms of

$$
\delta_{n l} \equiv \nu_{n i}-\nu_{n-1, i+2} \simeq-(4 l+6) \frac{\Delta \nu}{4 \pi^{2} \nu_{n l}} \int_{0}^{R} \frac{\mathrm{d} c}{\mathrm{~d} r} \frac{\mathrm{d} r}{r}
$$

where we neglected the term in the photospheric sound speed $c(R)$. This equation indicates that $\delta_{n l}$ is predominantly determined by conditions in the solar core. It should be noted, however, that the accuracy of equation (38) is questionable: it appears to agree fortuitously with frequencies computed for models of the present Sun, whereas it is less successful for models of different ages or masses (Gabriel 1989; Christensen-Dalsgaard 1991). In fact, the normal asymptotic approximation breaks down in the core where conditions vary rapidly, and the derivation of equation (34) neglected the perturbation in the grav!tational potential which, as we have seen, is important for low-degree modes. Nonetheless it remains true that $\delta_{n l}$ is a useful diagnostics of the structure of the core.

\subsection{Perturbation analysis of effects of model changes}

So far I have considered effects of solar structure only in terms of the asymptotic description of acoustic modes. To obtain more precise results, which in addition are valid for $f$ and $g$ modes, we must return to the original equations of adiabatic oscillations. In this way we obtain relations, precisely valid in the linear approximation, between changes in the structure and the corresponding frequency changes. In addition, the analysis provides further insight into aspects of the oscillations. 


\subsubsection{Reformulation of the oscillation equations}

A great deal of insight into the properties of adiabatic oscillations can be obtained by regarding the equations as an eigenvalue problem in a Hilbert space (Eisenfeld 1969; Dyson \& Schutz 1979; Christensen-Dalsgaard 1981). The starting point is the perturbed equations of motion (see the chapter by Leibacher). After separation of the time dependence as $\exp (-i \omega t)$, these can be written as

$$
\omega^{\prime 2} \delta \mathbf{r}=\mathcal{F}(\boldsymbol{\delta} \mathbf{r})
$$

where

$$
\mathcal{F}(\boldsymbol{\delta} \mathbf{r})=\frac{1}{\rho} \nabla p^{\prime}-\nabla \Phi^{\prime}-\frac{\rho^{\prime}}{\rho} \mathbf{g}
$$

Here $\delta \mathbf{r}$ is the displacement vector, $p^{\prime}, \rho^{\prime}$ and $\Phi^{\prime}$ are Eulerian perturbations of pressure, density and gravitational potential and $\mathrm{g}$ is the equilibrium gravitational acceleration. As indicated, $\mathcal{F}$ is a linear functional of $\boldsymbol{\delta} \mathbf{r}$. To see this, note that from the continuity equation,

$$
\rho^{\prime}+\operatorname{div}(\rho \boldsymbol{\delta} \mathbf{r})=0
$$

$f^{\prime}$ is a linear functional of $\delta \mathbf{r}$. The gravitational potential perturbation $\Phi^{\prime}$ may then be obtained by integrating the perturbed Poisson's equation. In the adiabatic case $p^{\prime}$ can be obtained directly from $\rho^{\prime}$ and $\delta \mathbf{r}$. This defines the adiabatic operator $\mathcal{F}_{a}$. The nonadiabatic case is more complicated, but here also it is possible to obtain $p^{\prime}$ as a linear functional of $\boldsymbol{r} \mathbf{r}$ (see Christensen-Dalsgaard 1981).

I now introduce a space $\mathcal{H}$ of vector functions of position in the star, with suitable regularity properties, and define an inner product on $\mathcal{H}$ by

$$
<\xi, \eta>=\int_{V^{\prime}} \rho \xi^{*} \cdot \eta \mathrm{d} V
$$

for $\xi, \eta$ in $\mathcal{H}$; here "*" denotes the complex conjugate, and the integration is over the volume $V$ of the star. I also introduce the domain $\mathcal{D}(\mathcal{F})$ of the operator $\mathcal{F}$ as those vectors in $\mathcal{H}$ satisfying the surface boundary condition that the Lagrangian pressure perturbation vanish. The central result is now that, as shown by Lynden-Bell \& Ostriker (1967), the operator $\mathcal{F}_{a}$ corresponding to equation (40) for adiabatic oscillations is symmetric, in the sense that

$$
\left.<\xi, \mathcal{F}_{a}(\boldsymbol{\eta})>=<\mathcal{F}_{a}(\xi), \boldsymbol{\eta}\right\rangle, \quad \text { for } \xi, \boldsymbol{\eta} \in \mathcal{D}(\mathcal{F})
$$

From equation (43) a number of useful properties of $\mathcal{F}_{a}$ follow immediately. The simplest result is that the squared eigenfrequencies are real. I introduce the functional $\Sigma$ on $\mathcal{D}(\mathcal{F})$ by

$$
\Sigma(\xi)=\frac{\left\langle\xi, \mathcal{F}_{a}(\xi)>\right.}{\langle\xi, \xi\rangle}
$$


it follows from equation $(43)$ that $\Sigma(\xi)$ is real. If $\omega_{0}^{2}$ is an eigenvalue of the problem with eigenvector $\xi_{0}$, i.e.,

$$
\mathcal{F}_{a}\left(\xi_{0}\right)=\omega_{0}^{2} \xi_{0}
$$

then

$$
\Sigma\left(\xi_{0}\right)=\omega_{0}^{2}
$$

and hence $\omega_{0}^{2}$ is real. Also, the eigenfunctions may be chosen to be real at all $r$.

As is well known, a second property of a symmetric operator is that eigenvectors corresponding to different eigenvalues are orthogonal. Thus if

$$
\mathcal{F}_{a}\left(\xi_{1}\right)=\omega_{1}^{2} \xi_{1} ; \quad \mathcal{F}_{a}\left(\xi_{2}\right)=\omega_{2}^{2} \xi_{2} ; \quad \omega_{1}^{2} \neq \omega_{2}^{2},
$$

then

$$
<\xi_{1}, \xi_{2}>=0
$$

A very important result concerns the effect of a small perturbation to the oscillation equations. This perturbation could result from a small change to the equilibrium model, to the inclusion of nonadiabatic effects (ChristensenDalsgaard 1981) or to the inclusion of the effect of large-scale velocity fields, such as rotation, in the model. I characterize the perturbation by a change $\delta \mathcal{F}$ in the operator defining the oscillation equations. If $\delta \mathbf{r}_{0}$ and $\omega_{0}$ are solutions to the adiabatic oscillation equations,

$$
\omega_{0}^{2} \delta \mathbf{r}_{0}=\mathcal{F}_{a}\left(\boldsymbol{\delta} \mathbf{r}_{0}\right)
$$

the change in $\omega^{2}$ caused by the perturbation $\delta \mathcal{F}$ can be obtained from first order perturbation analysis (e.g. Schiff 1949) as

$$
\delta \omega^{2} \simeq \frac{\left\langle\delta \mathbf{r}_{0}, \delta \mathcal{F}\left(\boldsymbol{\delta} \mathbf{r}_{0}\right)>\right.}{\left.<\delta \mathbf{r}_{0}, \delta \mathbf{r}_{0}\right\rangle}
$$

Thus the frequency change can be computed from the unperturbed eigenvector. Some consequences of this relation are discussed in the following sections.

\subsubsection{Effects of changes in solar structure}

As an example of the use of equation (50), we consider in more detail changes in the frequencies caused by changes in the equilibrium model. The effects on the oscillations of the change in the structure can be described as a perturbation $\delta \mathcal{F}_{a}$ in the operator characterizing adiabatic oscillations. According to equation (50), 
the corresponding relative freculency change. for a mode $(n, l)$ with sigenvector $\xi_{n !}$ in $\mathcal{H}$. is then

$$
\frac{\delta \omega_{n l}}{\omega_{n l}}=\frac{1}{2} \frac{\delta \omega_{n l}^{\prime 2}}{\omega_{n l}^{2}}=\frac{\left\langle\xi_{n l} . \delta \mathcal{F}_{n l}\left(\xi_{n l}\right)\right\rangle}{2 \omega_{n l}^{2}<\xi_{n l}, \xi_{n l}>} \text {. }
$$

11 is convenient in the following to express $\xi_{n l}$ in terms of its radial and horizontal amplitudes $\xi_{r, n l}(r)$ and $\xi_{\mathrm{t}, n l}(r)$ (assumed to be real) in a sphericalhamonic decomposition. chosen such that

$$
<\xi_{n l}, \xi_{n l}>=1 \pi \int_{0}^{R}\left[\xi_{r, n l}(r)^{2}+\xi_{\mathrm{h}, n l}(r)^{2}\right] r^{2} \rho \mathrm{d} r
$$

We can express this quantily in terms of a nomalized inertia $E_{n l}$, defined by

$$
E_{n l}=\frac{1 \pi \int_{0}^{K}\left[\xi_{r, n l}(r)^{2}+\xi_{\mathrm{h}, n l}(r)^{2}\right] \rho_{0} r^{2} \mathrm{~d} r}{M\left[\xi_{r, n l}(R)^{2}+\xi_{\mathrm{h}, n l}(R)^{2}\right]}=\frac{M_{\text {morde }}}{M}
$$

where $M$ is the total mass of the star, and $M_{\text {mode }}$ is the so-called modal mass. Also. we similarly represent $\delta \mathcal{F}_{a}$ on component form as

$$
\delta \mathcal{F}_{a l}\left(\xi_{n l}\right)=\left(\phi_{r}\left[\xi_{n l}\right], \phi_{\mathbf{h}}\left[\xi_{r l l}\right]\right),
$$

where $o_{r}\left[\xi_{n l}\right](r)$ and $\phi_{h}\left[\xi_{n l}\right](r)$ are functions of $r$. 'Then we can write equation (51) as

$$
E_{n l} \frac{\delta \omega_{n l}}{\omega_{n l}}=I_{n l}
$$

where

$$
I_{n l}=\frac{2 \pi \int_{0}^{R}\left[\xi_{r, n l}(r) \phi_{r}\left[\xi_{n l}\right](r)+\xi_{\mathrm{h}, n l}(r) \phi_{\mathrm{h}}\left[\xi_{n l}\right](r)\right] \rho r^{2} \mathrm{~d} r}{M \omega_{n l}^{2}\left[\xi_{r, n l}^{2}(R)+\xi_{h, n l}^{2}(R)\right]} .
$$

Thus $I_{n l}$ gives the integrated effect of the perturbation, normalized to the total photospheric displacement.

There is a close analogy between the exact equations (55) and (56) and the asymptotic expression (22). In both cases the factor multiplying $\delta \omega$ represents the fact that modes with larger inertia are more difficult to perturb. The effect on the frequencies of the change in the model is described by the right hand sides of equations (22) and (56), and depends on the overlap between the eigenfunction and the change in the model.

Equations (55) and (56) provide a somewhat formal linear relation between the change in the model and the change in the frequency. It follows from the discussion in Section 3.2 that the changes in the coefficients of the oscillation equations, and hence the changes $\phi_{r}\left[\xi_{n l}\right](r)$ and $\phi_{\mathrm{h}}\left[\xi_{n l}\right](r)$ in the components of $\delta \mathcal{F}_{a}$, can be expressed in terms of changes in two suitably chosen model variables, for example density and sound speed. For simplicity, I assume that the change 
in the model occurs without a change in its radius (this would in general be the case for models of the Sun, where the radius is known with high accuracy) and let $\delta \rho$ and $\delta c$ denote the differences in $\rho$ and $c$ between the equilibrium models, at fixed $r$. Then equations (55) and (56) can be expressed as

$$
\frac{\delta \omega_{n l}}{\omega_{n l}}=\int_{0}^{R}\left[K_{n l}^{\cdot(c, \rho)}(r) \frac{\delta c}{c}(r)+K_{n l}^{(\rho, c)}(r) \frac{\delta \rho}{\rho}(r)\right] \mathrm{d} r
$$

(e.g. Gough \& Thompson 1991), where the kernels $K_{n l}^{(c, \rho)}$ and $K_{n l}^{(\rho, c)}$ are computed from the eigenfunctions. Examples of such kernels are shown in Figure 3.

As implied by Section 3.2, the pair $(c, \rho)$ in equation (57) can be replaced by other suitable pairs, such as for example $\left(c, \Gamma_{1}\right)$ or $\left(\rho, \Gamma_{1}\right)$. Further transformations are possible if the equation of state and the heavy-element abundances are assumed to be known. Then $\Gamma_{1}$ is determined by $p, \rho$ and the helium abundance $Y$. Also, since $p$ can be obtained from $\rho$ under the assumption of hydrostatic equilibrium, $\delta \Gamma_{1}$ can be expressed in terms of just $\delta \rho$ and $\delta Y$. Here $\rho$ may be replaced by suitable quantities obtained from $\rho, p$ or their derivatives. A convenient parameter is $u=p / \rho$, related to the sound speed by $c^{2}=\Gamma_{1} u$. Thus equation (57) becomes

$$
\frac{\delta \omega_{n l}}{\omega_{n l}}=\int_{0}^{R}\left[K_{n l}^{-(u, Y)}(r) \frac{\delta u}{u}(r)+K_{n l}^{(Y, u)}(r) \frac{\delta Y}{Y}(r)\right] \mathrm{d} r ;
$$

here the kernels $K_{n l}^{(Y, u)}(r)$ are only of substantial magnitude in the regions where $\Gamma_{1}$ varies significantly, i.e., in the main ionization zones.

\subsubsection{Effects of sharply localized feature}

Equation (57) provides the general relation between differences between solar models and the corresponding frequency differences. We now assume that the Sun contains a sharply localized feature at some radius $r=i_{0}$; examples of such features are the sudden change in the slope of the sound-speed gradient at the base of the convection zone, or the rapid variation of $\Gamma_{1}$ in the second helium ionization zone. The effect of the feature on the frequencies can be estimated by introducing a suitably smoothed model which only differs from the actual model in the close vicinity of the feature (Monteiro, Christensen-Dalsgaard \& Thompson 1994); $\delta c$ and $\delta \rho$ are then taken to be the differences between the actual and the smoothed structure, such that they would be non-zero only near $r_{0}$. Hence the effects of the feature on the frequencies are essentially given by $K_{n l}^{(c, \rho)}\left(r_{0}\right)$ and $K_{n l}^{(p, c)}\left(r_{0}\right)$.

The behaviour of the frequency effects can be understood by noting that the kernels are, very roughly, proportional to the square of the eigenfunction. 


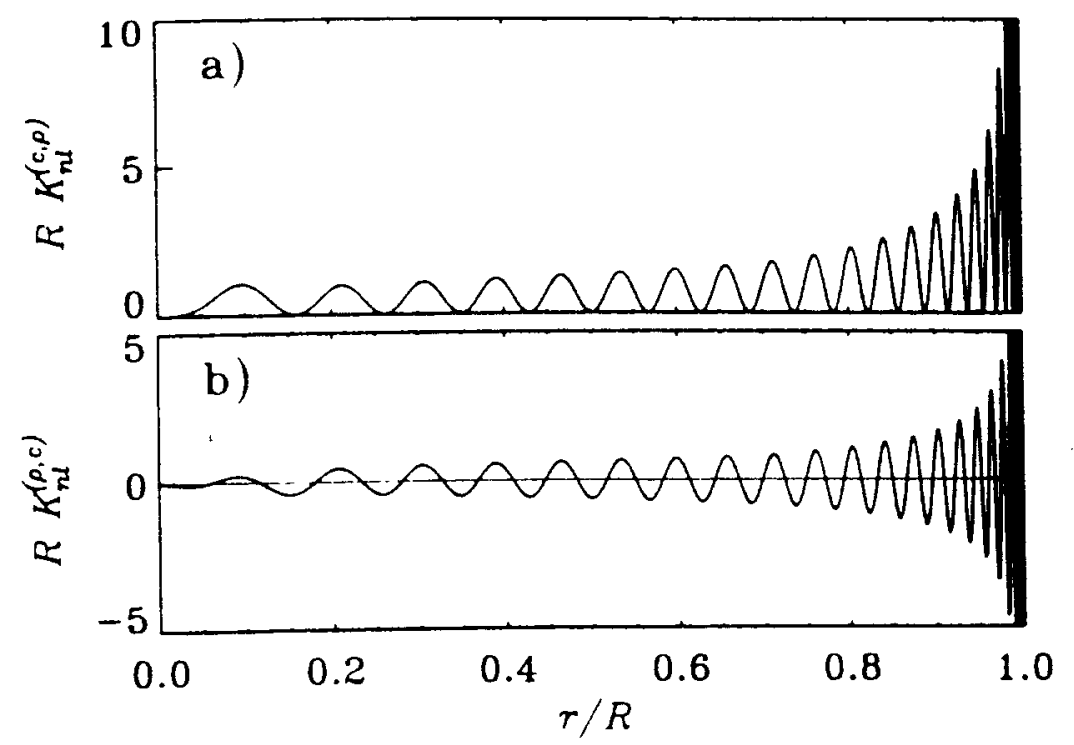

Figure 3: Kernels $R K_{n l}^{(c, o)}$ (panel a) and $R K_{n l}^{-(\text {p.c) }}$ (panel b) for an $l=1, n=21$ modt (with frequency $\nu=3.1 \mathrm{mHz}$ ) of a model of the present Sun. For clarity the plots have been truncated: the maximum value of $R K_{n l}^{(c, \rho)}$ is 45 , while the fxtreme values of $R K_{n l}^{(\rho, c)}$ are -24 and 16 .

For high-order acoustic modes one finds that the amplitude $\xi_{r}$ of the radial displacement depends on $r$ and $\omega$ roughly as

$$
\xi_{r} \sim \cos \left[\omega \tau-\left(\alpha+\frac{1}{4}\right) \pi\right]
$$

where

$$
\tau=\int_{r}^{R} \frac{\mathrm{d} r}{c}
$$

is the acoustical depth, and $\alpha$ is the phase function introduced in equation (12). Thus the kernels behave roughly as $\cos (2 \omega \tau+\phi)$ for some phase $\phi$. It follows that the frequency changes induced by a sharp feature oscillate as a function of frequency, with a "period" determined by the depth $\tau$ at which the feature is located: features localized near the surface give rise to frequency changes varying slowly with frequency, whereas deeper features cause more rapid variations (e.g. Thompson 1988; Vorontsov 1988; Gough 1990). This is illustrated in Figure 4, where kernels of low-degree modes have been plotted against frequency at fixed locations corresponding approximately to the base of the convection zone (Figure $4 a$ ), the second helium ionization zone (Figure $4 b$ ) and the photosphere (Figure 4c). 

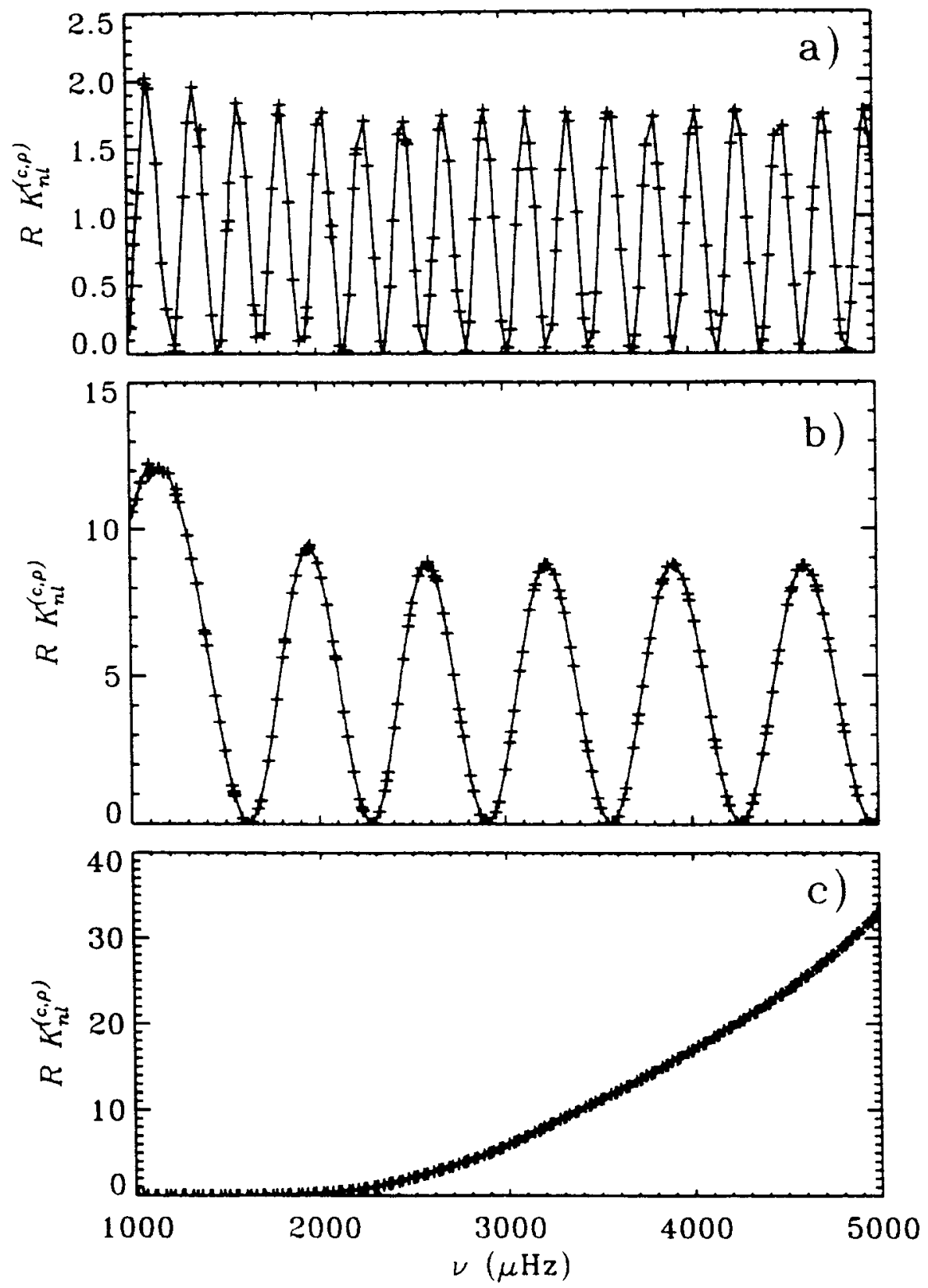

Figure 4: Kernels $K_{n l}^{(c, \rho)}\left(r_{0}\right)$ at $r_{0}=0.72 R$ (panel a) $r_{0}=0.98 R$ (panel b) and $r_{0}=R$ (panel $\mathrm{c}$ ), for modes of degree $l \leq 5$, plotted against frequency. For clarity the points have been connected. 


\subsubsection{Effects of near-surface modifications}

It was argued in Section 2.5 that the near-surface region introduces substantial uncertainty in the computation of oscillation frequencies. The effects of these errors on the frequencies (an be analyzed by means of an analogue of equations (5.) and (56), where now the perturbation $\delta \mathcal{F}$ represents incorrectly treated fertures in the model and the physics of the oscillations. These may include frrors in the hydrostatic structure, such as would be introduced by the neglect of turbulent pressure. as well as errors in the physics of the oscillations, e.g. the assumption of adiabaticity. I assume that $\delta \mathcal{F}$ is localized near the solar surface, in the sense that

$$
\phi_{i}[\xi](r) \simeq 0, \quad \phi_{\mathrm{h}}[\xi](r) \simeq \theta \quad \text { for } \quad R-r>\delta,
$$

for some small $\delta$. Thus in equation (56) for $I_{n d}$ the integration extends essentially only over the region $[R-\delta, R]$. Modes extcnding substantially more deeply, i.e., with $R-r_{\mathrm{t}} \gg \delta$, correspond to waves which propagate almost vertically in the region of modification: thus at a given frequency the eigenfunctions are essentially independent of $l$ in this region: as a result $I_{n l}$ depends little on $l$ at fixed $\omega$. The same is therefore true of $E_{n l} \delta \omega_{n l}$. To get a more convenient representation of this property, we introduce

$$
Q_{n l}=\frac{E_{n l}}{\bar{E}_{0}\left(\omega_{n l}\right)},
$$

where $E_{l}(\omega)$ is obtained by interpolating to $\omega$ in $E_{n l}$ at fixed $l$. Then $Q_{n l} \delta \omega_{n l}$ is independent of $l$, at fixed $\omega$. for modes such that $R-r_{\mathrm{t}} \gg \delta$. This behaviour may be used to identify, and eliminate, the effects of the near-surface uncertainties. $Q_{n l}$ has been plotted in Figure 5 for selected values of $l$. Its variation with $l$ is largely determined by the change in the penetration depth. Modes with higher degree penetrate less deeply and hence have a smaller inertia at given surface displacement. As a consequence of this their frequencies are more susceptible to changes in the model. Thus unscaled frequency differences resulting from near-surace effects are expected to show strong dependence on $l$. An example of this is discussed in Section 4.1 .

It should also be noticed that, according to trapping of the modes near the surface as determined by the behaviour of $\omega_{c}[\cdot f$. equations (30) and (31)] low-frequency modes are evanescent in the uncertain region, with much smaller amplitudes than in the interior. Thus for such modes the near-surface effects are expected to yield very small frequency changes. Finally, the discussion in Section 3.4.3 indicates that near-surface effects are likely to give rise to frequency changes varying slowly with frequency (see also Figure 4), unless, of course, the effects are themselves rapidly-varying functions of frequency. 

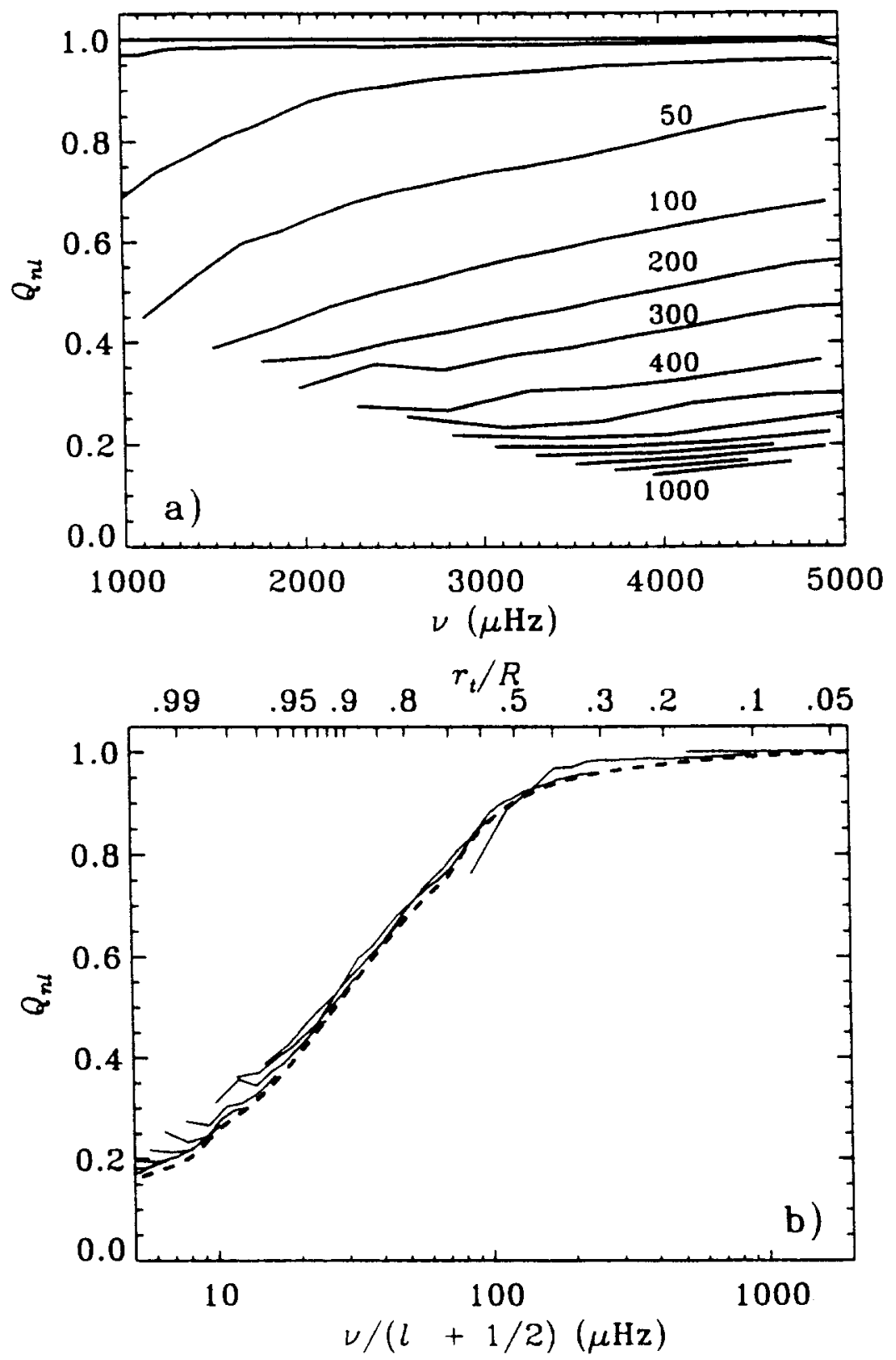

Figure 5: The solid lines show the inertia ratio $Q_{n l}$, defined in equation (62), for $p$ modes in a normal solar model. Each curve corresponds to a given degree $l$, In panel (a) $Q_{n l}$ is shown against frequency $\nu$, and selected values of $l$ are indicated. In panel (b) the lower abscissa is $\nu / L$, related to the turning-point radius $r_{\mathrm{t}}$ shown as the upper abscissa through equation (10). Here the heavy dashed curve shows the asymptotic scaling $\dot{S}_{n l} / \tau_{0}$ (see text). 
It follows from the preceding discussion that equation (57) should be replaced by

$$
\frac{\delta \omega_{n l}}{\omega_{n l}}=\int_{0}^{R}\left[K_{n l}^{-(c, \rho)}(r) \frac{\delta c}{c}(r)+K_{n l}^{(\rho, c)}(r) \frac{\delta \rho}{\rho}(r)\right] d r+Q_{n l}^{-1} \mathcal{G}\left(\omega_{n l}\right)
$$

where the function $\mathcal{G}(\omega)$ arcounts for the effect of the near-surface uncertainties. $\mathcal{G}$ must then be determined as part of the analysis of the frequency differcices. or suppressed by means of suitable filtering of the data. This equation is closely equivalent to equation (23), where the term $\mathcal{H}_{2}\left(\omega_{n l}\right)$ contains the contributions corresponding to $\mathcal{G}(\omega)$ Furthermore. $Q_{n l}$ corresponds asymptotically to $S_{n l}$, apart from a constant scaling factor. Indeed, in Figure $5(b)$ the dashed line shows $\hat{S}_{n l} / \tau_{0}$; hęre $\dot{S}_{n l}$ is defined as in equation (19), but excluding the term in $\mathrm{d} \alpha / \mathrm{d} \omega$, and $\tau_{0}$ is the limit of $S_{n l}$ for $r_{t} \rightarrow 0$. corresponding to the acoustical radius of the star evaluated from equation $(60)$, with $r=0$.

The arguments presented here assumed that the eigenfunctions of the modes were essentially independent of degree in the region of modification. This ceases to be true at sufficiently high $l$, leading to departures from the simple frequency dependence of the scaled frequency differences. Antia (1995) showed 'hat even for moderate degree the resulting $l$-dependence may be comparable with the observational errors so that it might affect the results of inversion. Procedures to hanclle such effects have been developed on the basis of higher-order asymptotic treatments, such as equation (33) ( $6 . g$. Gough \& Vorontsov 1995).

\section{APPLICATION TO MODELS AND OB- SERVED FREQUENCIES}

It was argued in Section 1.2 that the principal interest in the forward problem lies in the connection between the physics of solar models and their frequencies. To explore this connection, the present section considers various examples of physics modifications and their effects on the models and frequencies. The results are interpreted in terms of the properties of the oscillations presented in Section 3, particularly the asymptotic frequency changes discussed in Section 3.3.3. Furthermore, I briefly consider some aspects of the observed frequencies. More extensive results from the observations are presented in Section 5.

The results discussed here are largely based on complete solar models, obtained from evolution calculations starting at chemically homogeneous zero-age main-sequence models. These have been calibrated to have solar radius and luminosity, by adjusting the composition, characterized by the initial helium abundance $Y_{0}$, and a parameter describing convection ( $c f$. Section 2.2). To save 

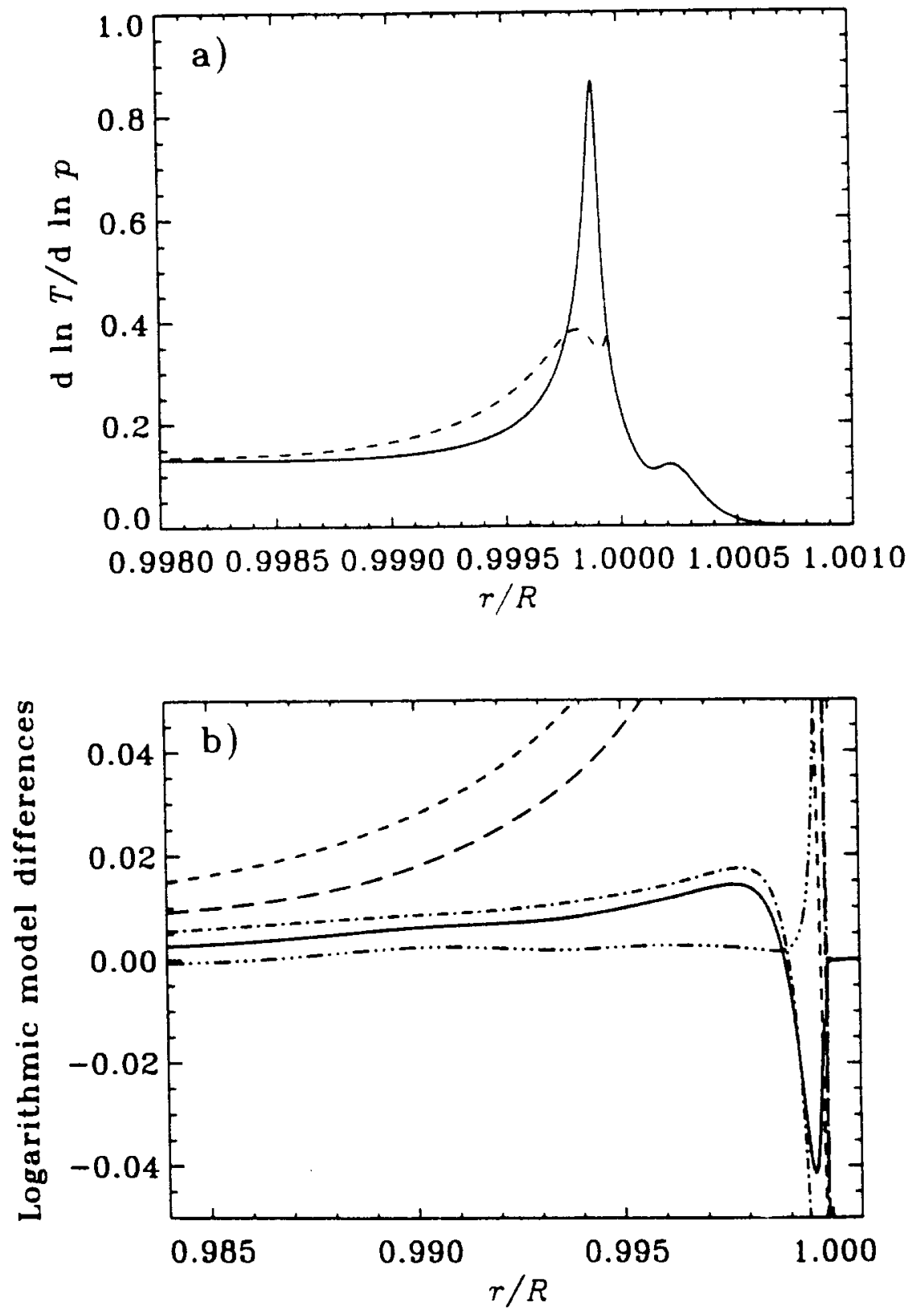

Figure 6: (a) $\nabla=\mathrm{d} \ln T / \mathrm{d} \ln p$ for a mixing-length model (solid line) and a modified model (dashed line). (b) Logarithmic differences at fixed radius $r$ between the modified model and the mixing-length model, in the sense (modified) - (mixing length). The following line styles have been used: $\delta \ln c:-; \delta \ln p$ : $\ldots . . . . . . . . ; \delta \ln \rho:-{ }_{0}$ 
computational effort. an alternative is to consider static models of the present Sum: here the abundance profile is based on scaling the hydrogen-abundance profile $\mathcal{X}(m)$ for a given reference model, as a function of mass $m$, by a factor $\chi$ such as to obtain the correct huminosity. This is often adequate to obtain insight into effects of modifications to the physics, as long as these occur outside the cnergy-generating core. Finally, when studying the properties of the convection zone, it is conveniont to consider models of the solar envelope alone. In such models, the surface huminosity and radius are chosen to have solar values and the composition is typically assumed to be constant. Also, the convection parameter is typically adjusted such that the model has a prescribed depth of convection zone. For physics modifications that are largely confined to the convection zone, such as those resulting from modifying the equation of state, this ensures that the radiative interior is approximately unchanged.

\subsection{Effects of changes in the superadiabatic region of the convection zone}

To illustrate the effects of near-surface uncertainties, I first consider a model where the treatment of the superadiabatic gradient has been artificially modified [see (hristensen-Dalsgaard (1986) for details]. In Figure 6, panel (a) compares the resulting $\nabla$ with the result of using mixing-length theory, whereas panel $(b)$ shows differences, at fixed $r$, between various quantities in the modified and the reference models. Here static models of the present Sun were used. The effects are confined to the outermost parts of the convection zone; the deeper parts of the convection zone and the radiative interior are virtually unchanged.

Frequency differences between the modified and the reference model are illustrated in Figure 7 . Panel (a) shows the original differences, which clearly depend strongly on both degree and frequency. It was argued in Section 3.4.4 that the $l$-dependence should be mainly associated with the variation of the mode inertia. This is confirmed by panel $(b)$ where the frequency differences have been scaled by the normalized inertia $Q_{n l}$ defined in equation (62). The scaled differences are virtually independent of degree for $l \$ 300$, corresponding to modes that propagate essentially vertically in the region where the model is modified. It should also be noticed that, in accordance with the discussion in Section 3.4.4, the differences are very small at low frequency and vary slowly with frequency.

This example illustrates the usefulness of scaling frequency differences to highlight effects of near-surface errors in the models. Thus in the following I shall almost exclusively consider differences scaled in this manner. 

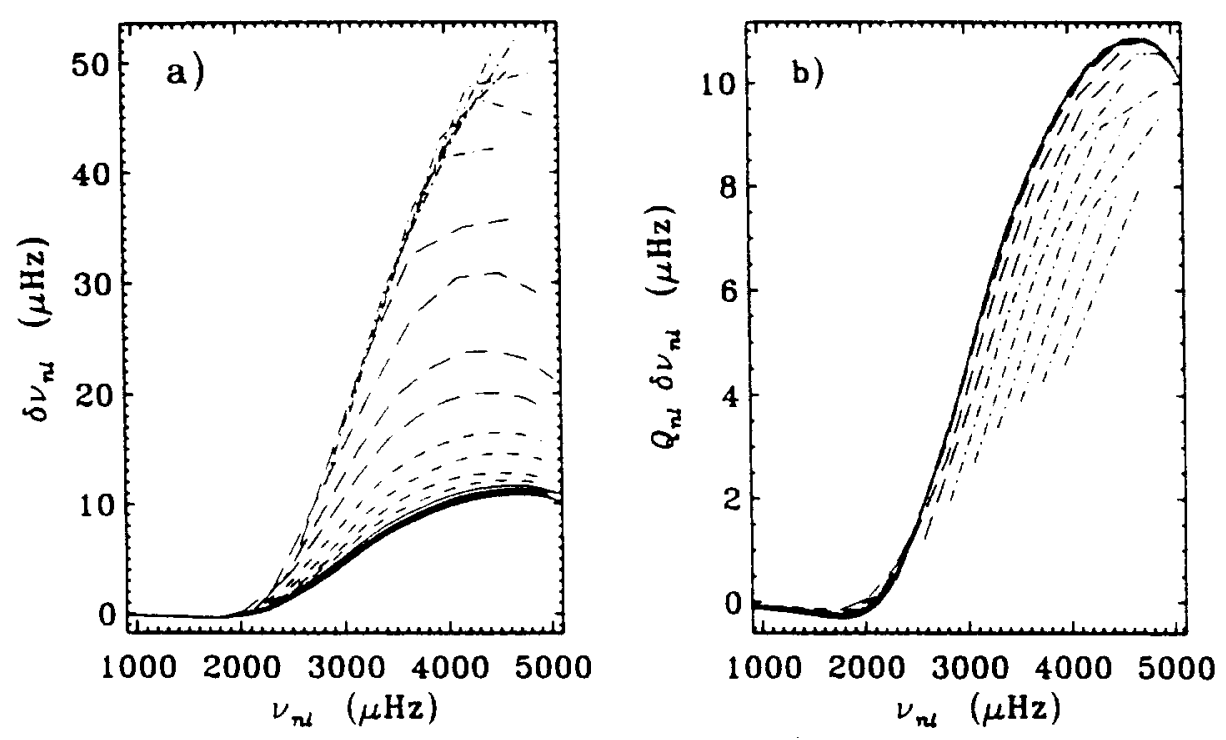

Figure 7 : Unscaled (panel a) and scaled (panel b) frequency differences corresponding to the model differences illustrated in Figure $6(\mathrm{~b})$. Modes of the same degree $l$ have been connected, according to the following line styles: $l=$ $0-30:-; l=40-100: \ldots \ldots \ldots . .+l=150-400:--.---$; $l=500-1100:-\cdots \cdot \ldots$.

The behaviour of $\nabla-\nabla_{\text {ad }}$ illustrated in Figure 6 was chosen with no physical basis. However, as noted in Section 2.3.1 refined versions of mixing-length theory have suggested that $\nabla-\nabla_{\text {ad }}$ might be sharper and higher than in mixinglength models. Figure 8 illustrates an example of such a model and the resulting scaled frequency differences; it is based on a parametrization by Monteiro et al. (1995b) of the convection treatment developed by Canuto \& Mazzitelli (1991). Within the range of degrees considered, between 20 and 300 , the scaled differences are essentially independent of degree, as shown $b$ : the small scatter of the points in the plot. Also, as discussed in Section 5.3, the frequency differences for $\log _{10}\left(\beta_{c}\right)=3$ bear a striking resemblance to the differences between the observed frequencies and those of a model computed with the normal mixing-length theory.

\subsection{Opacity increase near the base of the convection zone}

The effects of modifications of the physics of the radiative interior may be illustrated by considering changes in the opacity. Indeed, as mentioned in Section 2.5 , the opacity is likely to be a dominant source of uncertainty in the deep interior of the model. Although the actual error in the opacity is likely to be 

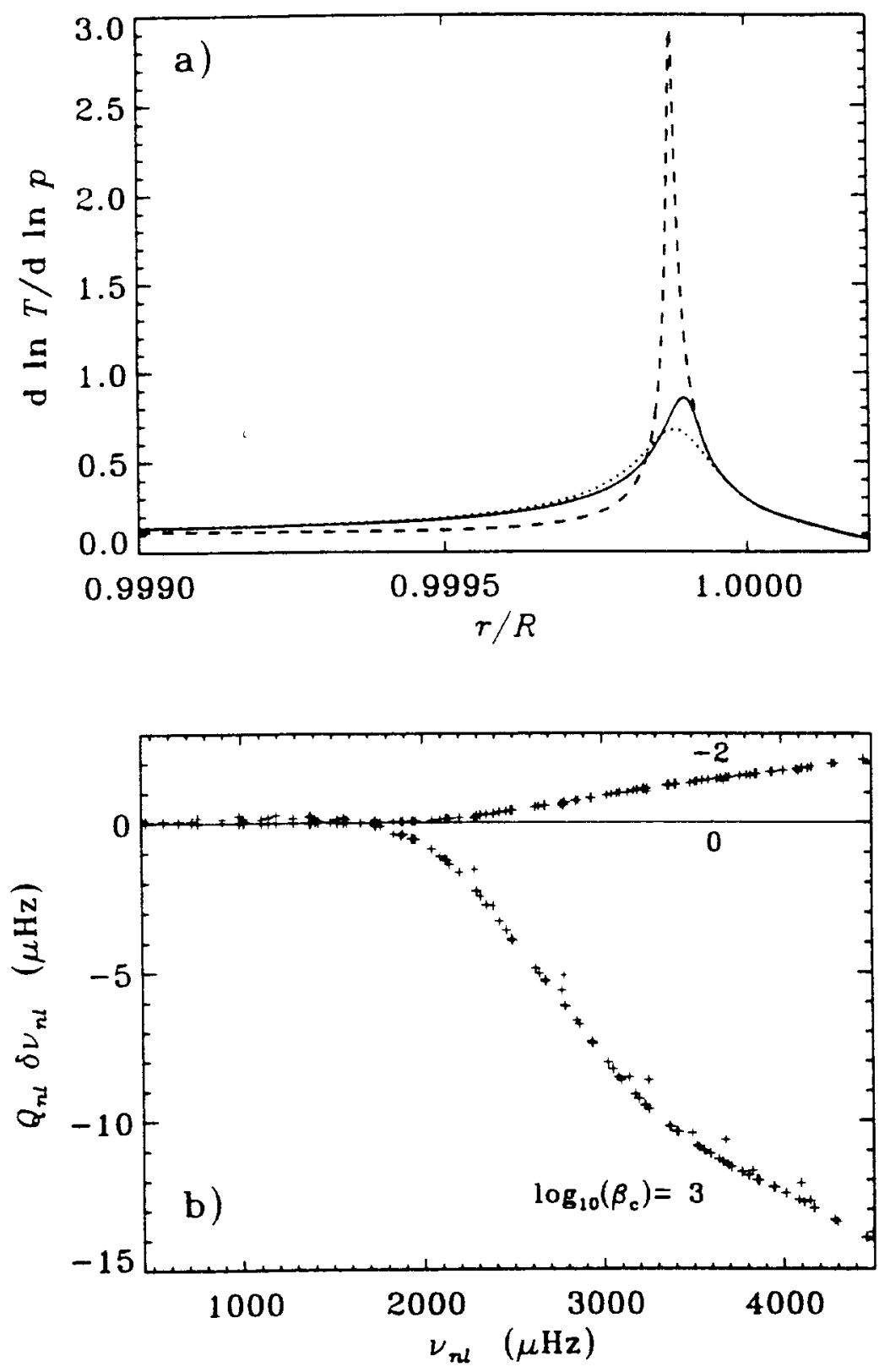

Figure 8: (a) $\nabla=\mathrm{d} \ln T / \mathrm{d} \ln p$ for three different treatments of convection, characterized by the parameter $\beta_{\mathrm{c}}$. Results are shown for a mixing-length model $\left(\beta_{\mathrm{c}}=1\right.$; solid line), a model approximating the Canuto \& Mazzitelli (1991) formulation $\left(\beta_{\mathrm{c}}=10^{3}\right.$; dashed line), and a model with lower and broader $\nabla-\nabla_{\text {ad }}$ $\left(\beta_{c}=10^{-2} ;\right.$ dotted line $)$. (b) Scaled frequency differences, relative to the mixinglength model, for $l=20,30,40,50,100,200,300$ for the models shown in panel (a). The points have been identified by the value of $\log _{10} \beta_{\mathrm{c}}$. (Adapted from Monteiro et al. 1995a). 
a complicated function of temperature and density, the effects are most easily understood by analyzing the consequences of a localized increase. The analysis is based on static models with scaled hydrogen abundance.

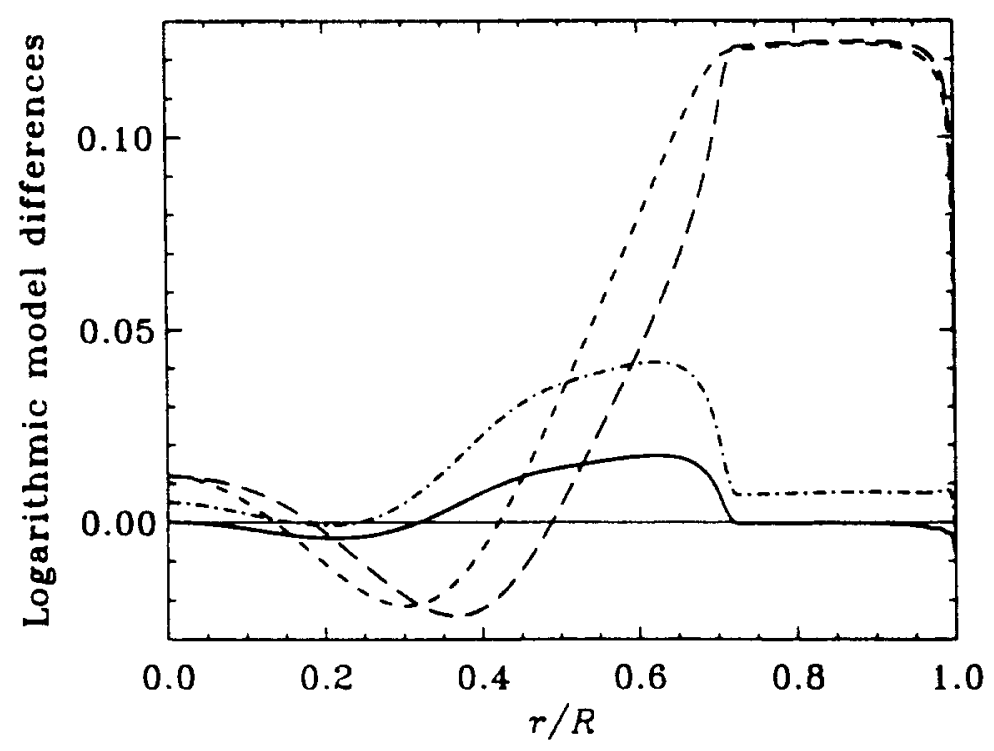

Figure 9: Logarithmic differences at fixed radius $r$ between the model with modified opacity and the reference model, in the sense (modified) - (reference). The following line styles have been used: $\delta \ln c:-; \delta \ln p: \ldots . . . . . . .$. $\delta \ln \rho:------; \delta \ln T:-\cdot-\cdot-\cdot-$

I consider an increase in the Rosseland mean opacity $\kappa$ defined as a function of temperature by

$$
\delta \log \kappa=A_{\kappa} \exp \left[-(y / \Delta \log T)^{2}\right]
$$

where

$$
y= \begin{cases}x-\log T_{1} & \text { for } x<\log T_{1} \\ 0 & \text { for } \log T_{1} \leq x \leq \log T_{2} \\ x-\log T_{2} & \text { for } x>\log T_{2}\end{cases}
$$

$\log$ being the logarithm to base 10. I confine the change near the base of the convection zone, by choosing $\log T_{1}=6, \log T_{2}=6.6$ and $\Delta \log T=0.15$, and take the maximum change to be $A_{\kappa}=0.1$. The resulting changes in sound speed, pressure and density are shown in Figure 9 . The corresponding frequency changes are largely determined by the the change in the sound speed. This is dominated by the increased depth of the convection zone in the modified model, resulting directly from the increase in the opacity: since the gradients of temperature and sound speed are steeper in the convection zone than in the radiative region below, there is a region where the sound speed increases more rapidly with depth in the modified model; therefore, the sound speed is 
sharply higher in the modified model just beneath the convection zone, as seen in the figure. In contrast, the sound-speed difference is very small in the bulk of the convection zone: this is consistent with equation (4) according to which the sound speed in this region, at given $r$, depends little on the details of the structure. The only visible exception is in the ionization zones near the surface, where the changes in composition and mixing length required to calibrate the modified model cause small additional differences in the sound speed.

The corresponding scaled frequency differences are shown in Figure $10(a)$. 'They clearly' reflect the behaviour of the sound-speed difference and the region 1.) which the modes are confined, as determined by the location of the turning point $r_{1}(c f$. equation 10). For low-degree modes which penetrate well beyond the hase of the convection zonte the frequencies are increased by the sound-speed increase in the outer parts of the radiative region. In contrast, high-degree modes are trapped in the convertion zone and are dominated by the small negative sound-speed differences in the ionization zones. For $l=20-50$ the behaviour depends strongly on frequency: higher-frequency modes perietrate more deeply, according to equation (10), and hence sense the positive sound-speed clifference below the convection zone. whereas low-frequency modes are largely confined to the convection zone.

The preceding discussion indicates the close link between the frequency differences and the location of the turning point. This is clearly in accordance with the asymptotic relation, equation (23), between the sound-speed difference and the frequency differences. It becomes obvious when, as in Figure $10(b)$, scaled relative differences are plotted against $\nu / L$ which according to equation (10) determines $r_{1}$. It is obvious that the general behaviour of the frequency differences is indeed dominated by $\mathcal{H}_{1}(\omega / L)$, with a sharp transition where the turning-point position $r_{\mathfrak{t}}$ of the modes coincides with the base of the convection zone. at $r \simeq 0.72 R$. For modes trapped in the convection zone, the frequencydependent term $\mathcal{H}_{2}(\omega)$ dominates; this comes predominantly from the negative sound-speed difference (hardly visible in Figure 9) in the hydrogen ionization zone, and hence varies slowly with frequency. A similar contribution is visible for the deeply-penetrating modes; here an additional rapid variation with frequency is induced by the sharp difference at the base of the convection zone ( $c f$. Section 3.4.3).

\subsection{Diffusion and settling of helium}

Helium diffusion and settling cause significant changes in the abundance profile of models of the present Sun and hence in the structure and frequencies of the models. Figure 11 shows differences hetween a model with diffusion and a normal 


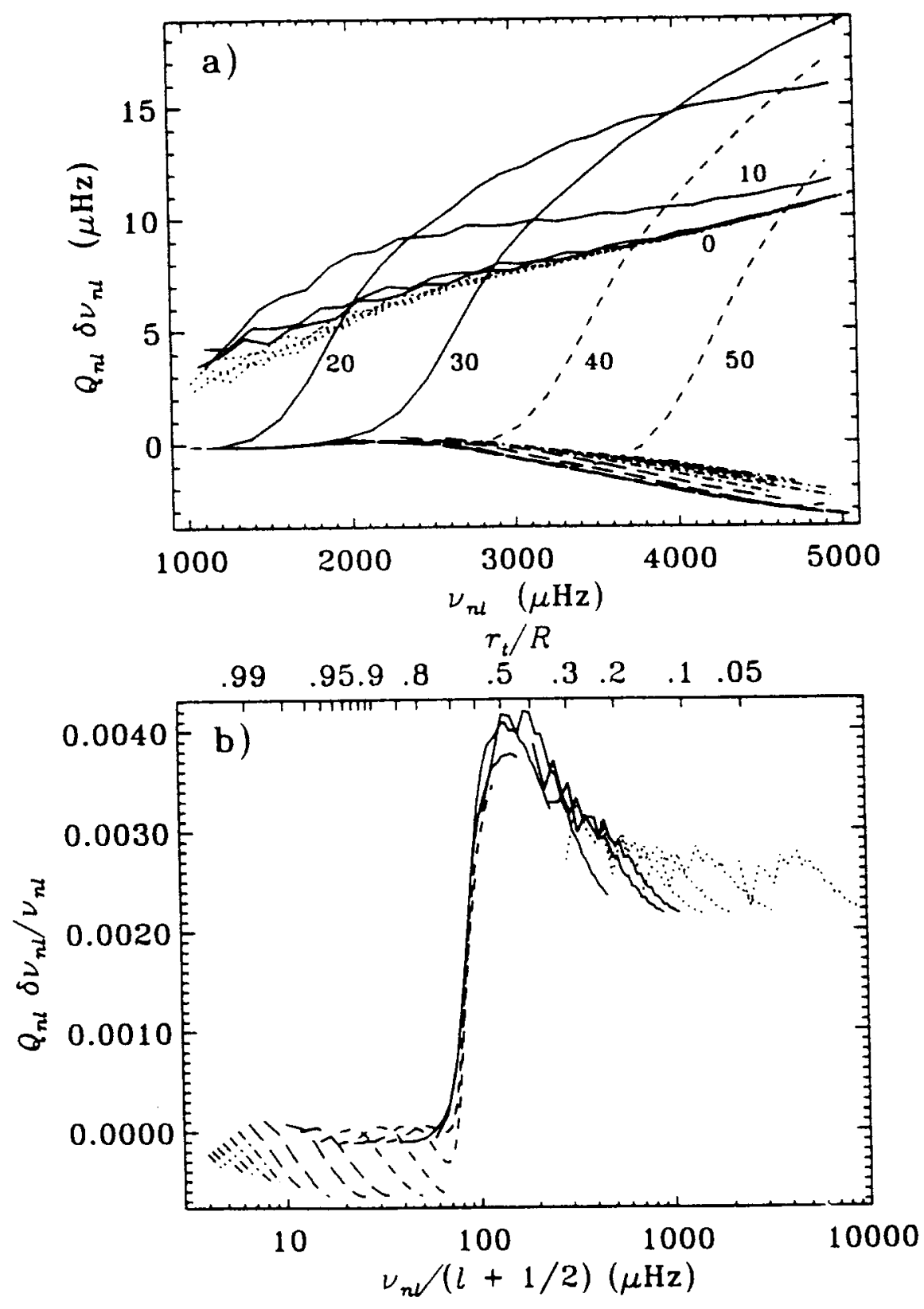

Figure 10: Scaled frequency differences corresponding to the model differences, resulting from opacity increase near the base of the convection zone, illustrated in Figure 9. Modes of the same degree / have been connected, according to the following line styles: $l=0-3: \ldots \ldots \ldots+\cdots, l=4-30:-; l=40-100$ :

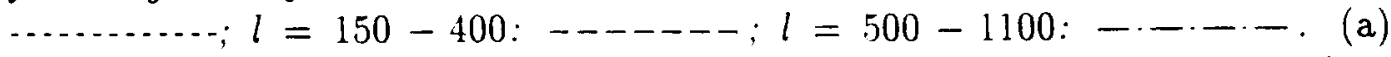
Absolute differences, plotted against frequency. Selected values of 1 have been indicated. (b) Relative differences, plotted against $\nu / L$ (lower abscissa) and the corresponding turning-point position [cf. equation (10); upper abscissa]. 
solar model (Christensen-I)alsgaard et al. 1993). Settling of helium causes an increase in the hydrogen abundance by about 0.03 in the convection zone, with a sharp gradient at its hase. In the outer parts of the radiative interior $X$ is still somewhat higher than in the normal model, while the central abundance is reduced. as a result of the accumulation of helium. The increase in $X$ near the hase of the convection zone leads to an increase in the clepth of the convection zone. and hence a substantial increase in sound speed in this region, just as in the case of the opacity increase discussed in Section 4.2. As in that case $\delta c / c$ is small in most of the convertion zone, whereas the change in composition results in a considerable change in $c$ in the ionization zones of hydrogen and helium, due to the change in $\Gamma_{1}$.

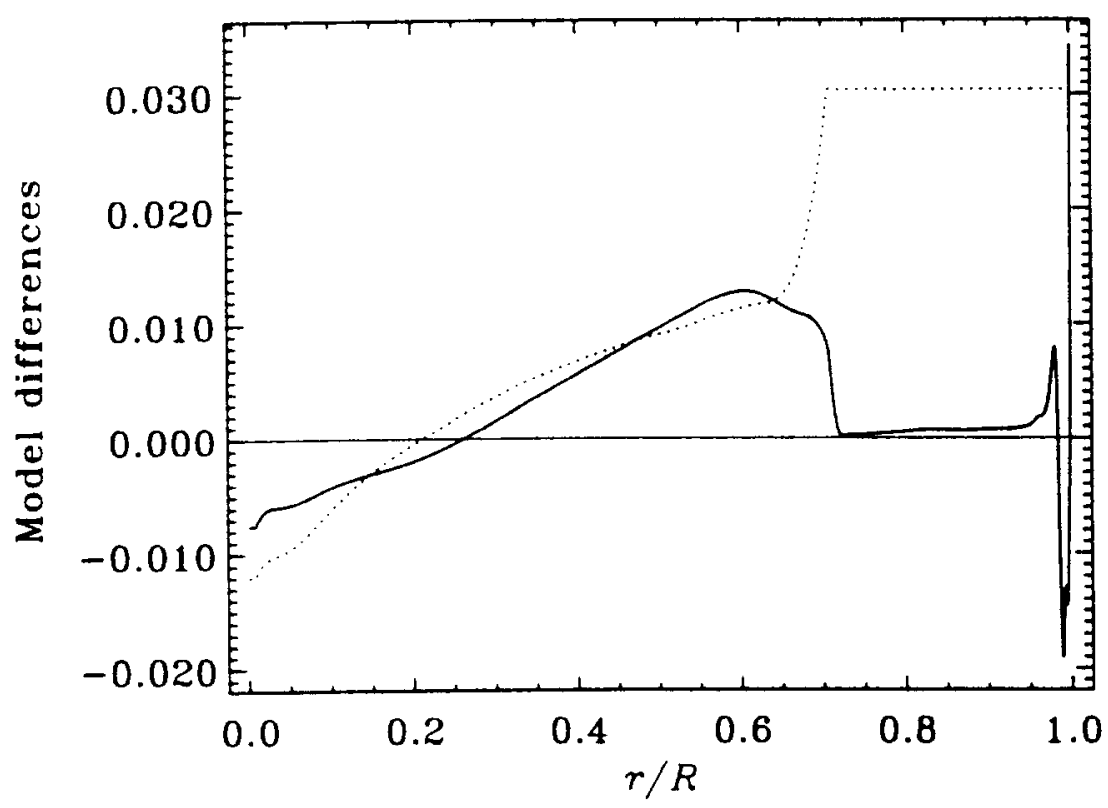

Figure 11: Differences, at fixed $r$, between a model of the present Sun including diffusion and gravitational settling of helium and a normal model without diffusion. The dotted line shows the difference $\delta X$ in the hydrogen abundance, and the continuous line shows the fractional difference $\delta c^{2} / c^{2}$ in squared sound speed. Adapted from Christensen-Dalsgaard et al. (1993).

As in the case of the opacity increase discussed in Section 4.2 the change in the frequencies is largely controlled by the location of the lower turning point. Thus Figure 12(a) shows scaled frequency differences, at selected values of $l$, between the diffusive and non-diffusive models, plotted against $\nu / L$ (with $L=l+1 / 2$ ). Here the scaling has been done in terms of the asymptotic factor $S_{n l}$, normalized by $\tau_{0}$, such that it tends to unity at low degree (note that, as indicated by Figure $5, S_{n l} / \tau_{0}$ is closely equivalent to $Q_{n l}$ ). Hence the 

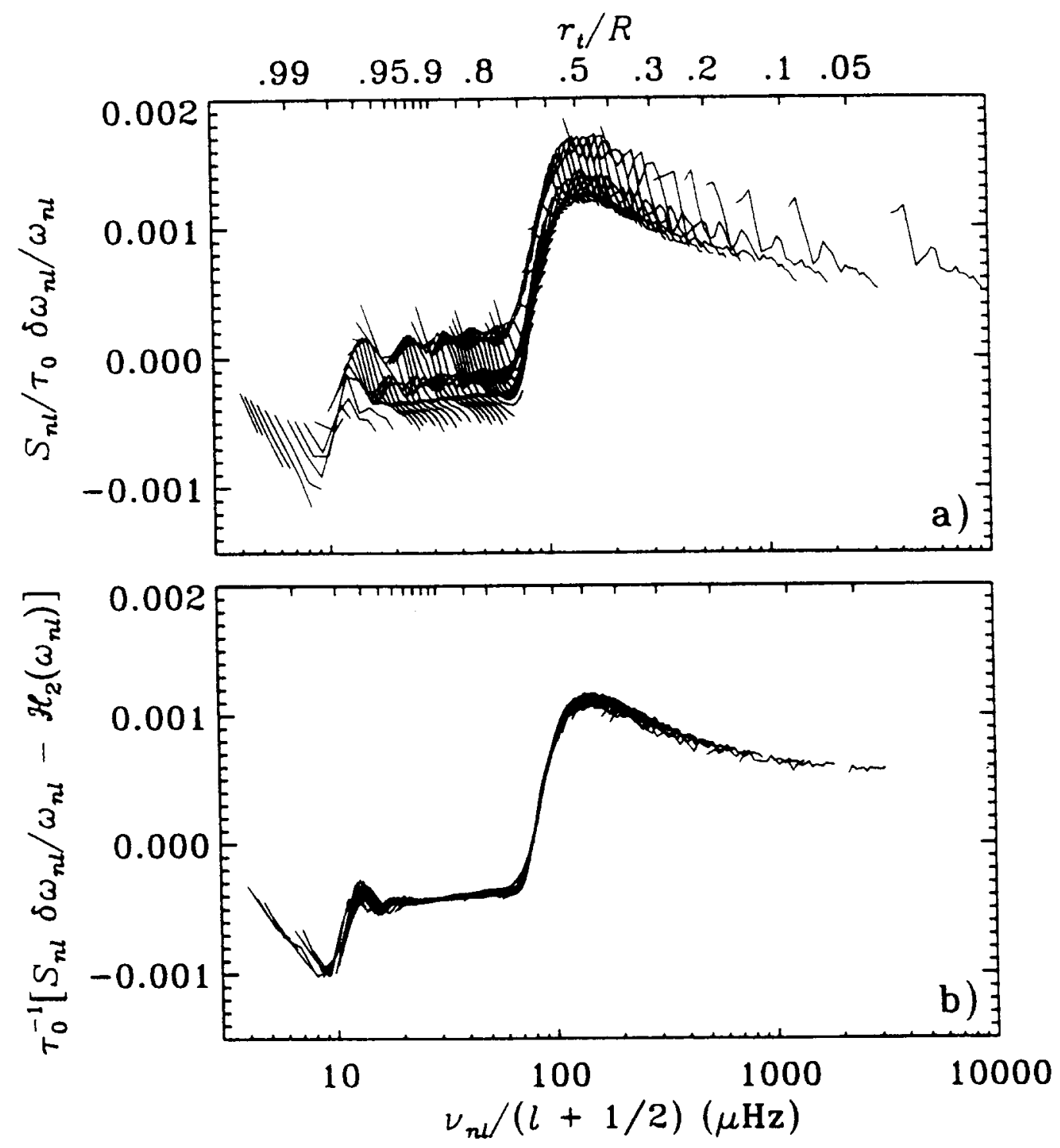

Figure 12: Scaled frequency differences corresponding to the model differences shown in Figure 11, plotted against $\nu /(l+1 / 2)$. The upper abscissa shows the location of the lower turning point, which is related to $\nu /(l+1 / 2)$ through equation (10). Points corresponding to fixed l have been connected. (a) Original scaled frequency differences. (b) Scaled differences, after subtraction of the function $\mathcal{H}_{2}(\omega)$ obtained from the spline fit. 
scaled frequency differences correspond in magnitude to the differences for lowregree modes. As in Figure $10(b)$ the dependence of $S \delta \nu / \nu$ on $\nu / L$ is dominated by a substantial positive sound-speed difference at the base of the convection zonc: modes with $\nu / L>100 \mu \mathrm{Hz}$ sense this feature and hence display a posilive freculuency difference; in contrast, for $\nu / L \lesssim 100 \mu \mathrm{Hz}$ the modes are entirely trapped in the convertion zone. and the frequency difference corresponds to the term $\mathcal{H}_{2}(l)$ arising from differences near the surface, particularly the difference in $X$.

This qualitative description suggests that the frequency differences may be analyzed in detail in terms of equation (23). To do so, I have determined the functions $\mathcal{H}_{1}$ and $\mathcal{H}_{2}$ by moans of the spline fit of Christensen-Dalsgaard et al. (1989), where details aboul the fitting method may be found. Briefly, the procedure is to approximate $\mathcal{H}_{1}(\omega / L)$ and $\mathcal{H}_{2}(\omega)$ by splines, the coefficients of which are determined through a least-squares fit to the scaled frequency differences. The knots of the splines in $w \equiv \omega / L$ are distributed uniformly in $\log w$ over the range considered, whereas the knots for the $\omega$-splines are uniform in $\omega$. I used $2 s$ knots in $w$ and 20 knots in $w$. Figure $12(b)$ shows the result of subtracting the function $\mathcal{H}_{2}(\omega)$ so obtained from the scaled frequency differences. It is evident that what remains is in fact very nearly a function of $\omega / L$ alone, directly reflecting the behaviour of $\delta \% / c$, as discussed above.

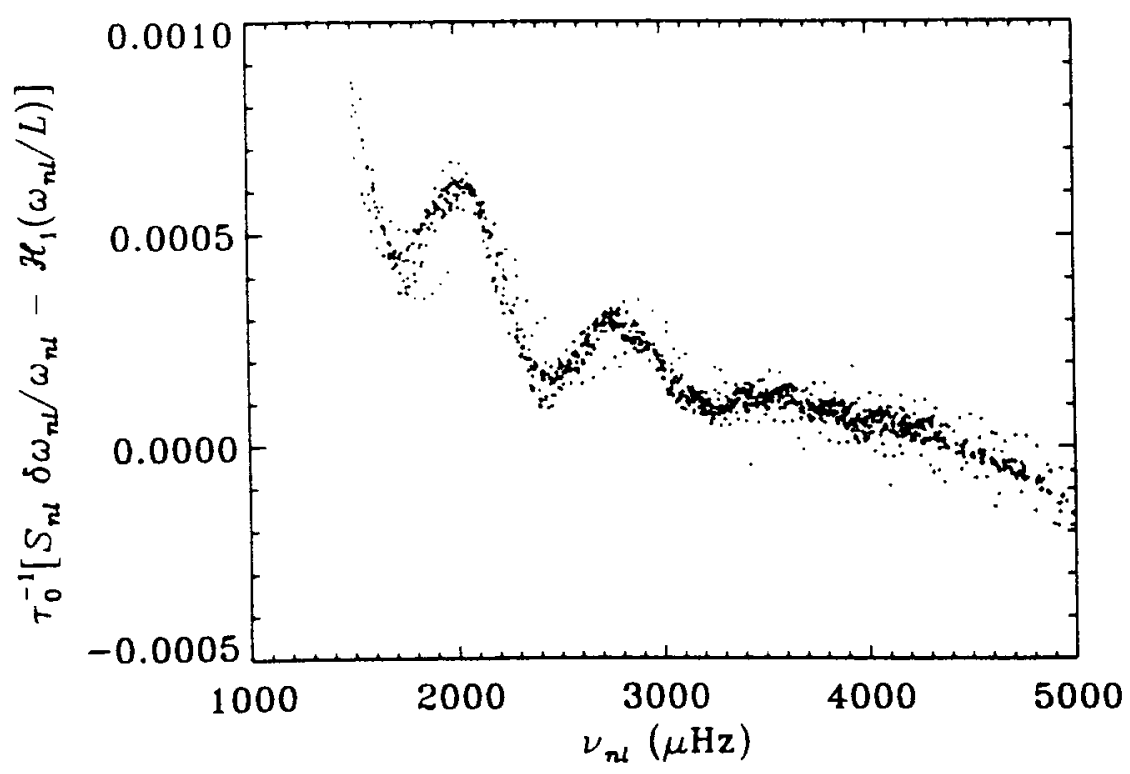

Figure 13: Scaled frequency differences corresponding to the model differences shown in Figure 11, after subtraction of the function $\mathcal{H}_{1}(\omega / L)$ resulting from the spline fit. 
The residual scaled frequency differences after subtraction of the term in $\mathcal{H}_{1}(\omega / L)$ are shown in Figure 13. These are clearly largely a function of frequency, although with some scatter. The behaviour is dominated, at low frequency, by an oscillation with a 'period' of around $800 \mu \mathrm{Hz}$. According to Figure 4 this corresponds to the effect of a sharp feature located around $r \simeq 0.98 R$, i.e., at the second helium ionization zone: it is caused by differences in the ionization zone resulting from the difference in helium abundance. The remaining slow trend is associated with changes in the hydrogen ionization zone and the atmosphere.

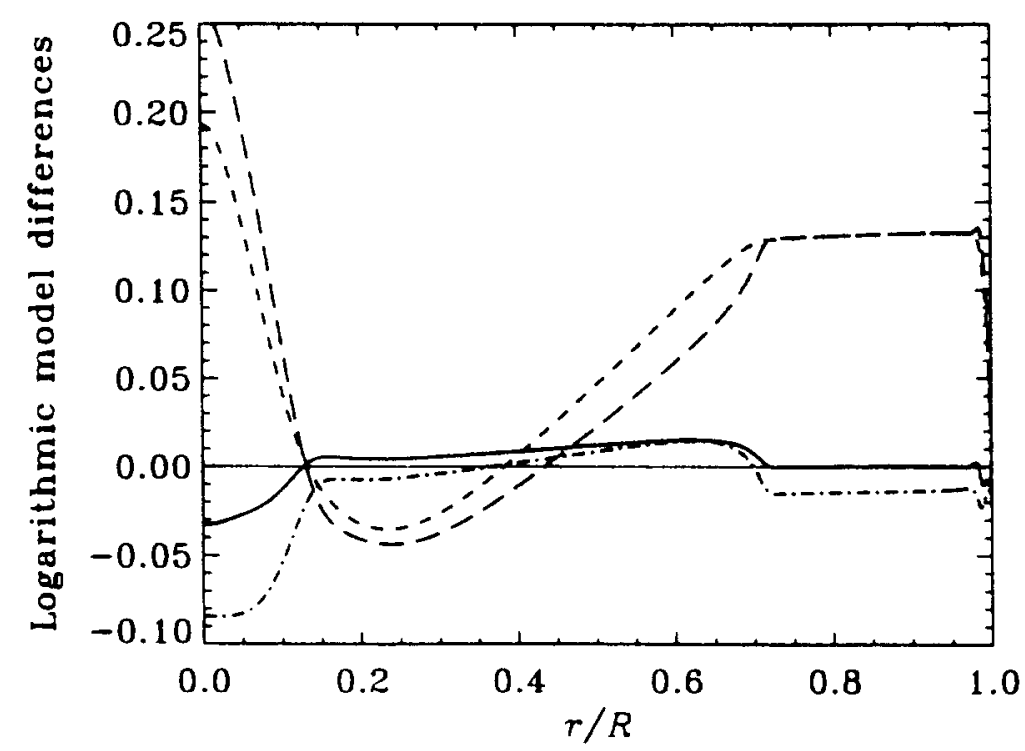

Figure 14: Logarithmic differences at fixed radius $r$ between the model with reduced central opacity and the reference model, in the sense (modified) - (reference). The following line styles have been used: $\delta \ln c:-; \delta \ln p$ : ............; $\delta \ln \rho:--\cdots---; \delta \ln T:-\ldots .-$.

\subsection{Opacity decrease in the core}

The solar neutrino problem (see the chapter by Bahcall) has motivated a number of suggestions for modifications to solar models designed to reduce the flux of high-energy neutrinos, by reducing the core temperature of the Sun. One such suggestion involved postulating the presence in the Sun of Weakly Interacting Massive Particles (WIMPs) whose motion was assumed to contribute to the energy transport. In this way the temperature gradient required for radiative transport, and hence the central temperature, could be reduced (Steigman et al. 1978; Spergel \& Press 1985; Faulkner \& Gilliland 1985). 


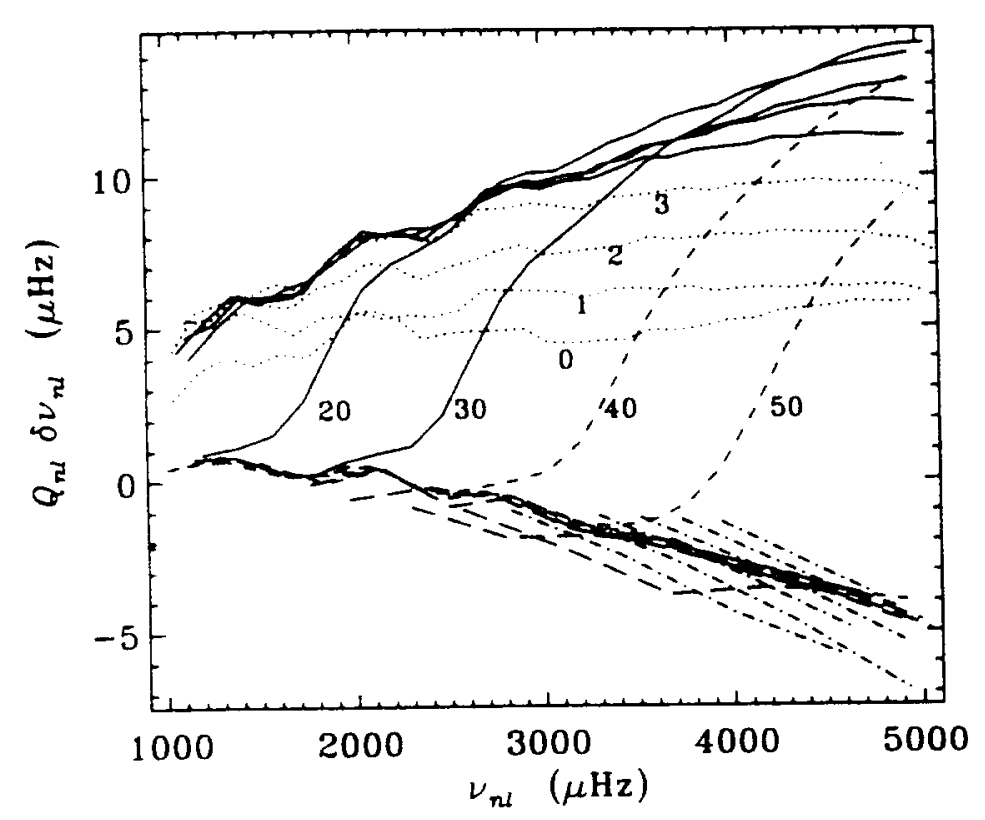

Figure 15: Scalcd frequency differences corresponding to the model differences, resulting from opacity decrease in the core, illustrated in Figure 14. Modes of the same degret l have becn connected, according to the following line styles:

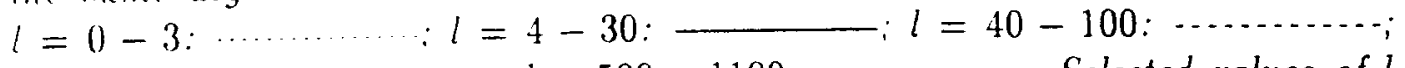
$l=150-400:-\ldots-\ldots: l=500-1100:-\ldots+\ldots$. Selected values of $l$ hane been indicated.

Here 1 model this effect through a reduction in the core opacity (see also (Christensen-Dalsgaard 1992). Specifically, the opacity was modified as in equations (64) and (65), but with $A_{\kappa}=-0.4, \log T_{1}=7.1, \log T_{2}=7.5$ and $\Delta \log T=0.04$. The resulting model differences, based on scaled static models, are shown in Figure 14. Clearly the central temperature has been reduced, leading to a decrease in the neutrino flux. This is accompanied by a reduction in the central sound speed and a dramatic increase in the core pressure and density. The changes in composition and mixing length required to obtain the correct luminosity and radius induce additional modifications in the outer parts of the model, including a modest increase in the depth of the convection zone, visible in the sound-speed difference.

Scaled frequency differences between the models are shown in Figure 15. The positive sound-speed difference just below the convection zone leads the the now familiar variations for the modes penetrating beyond the base of the convection zone. However, in addition the strong variations in the core cause a substantial dependence of the frequency differences on $l$, amongst the low-degree modes. This arises both from the depression of the sound speed and the increase in 
density; according to equation (29) the latter modification increases the effect of the perturbation in the gravitational potential and hence contributes to decreasing the frequencies. The resulting negative contributions to the frequencies are largest for the lowest-degree modes, leading to the variation in $\delta \nu$ with $l$.

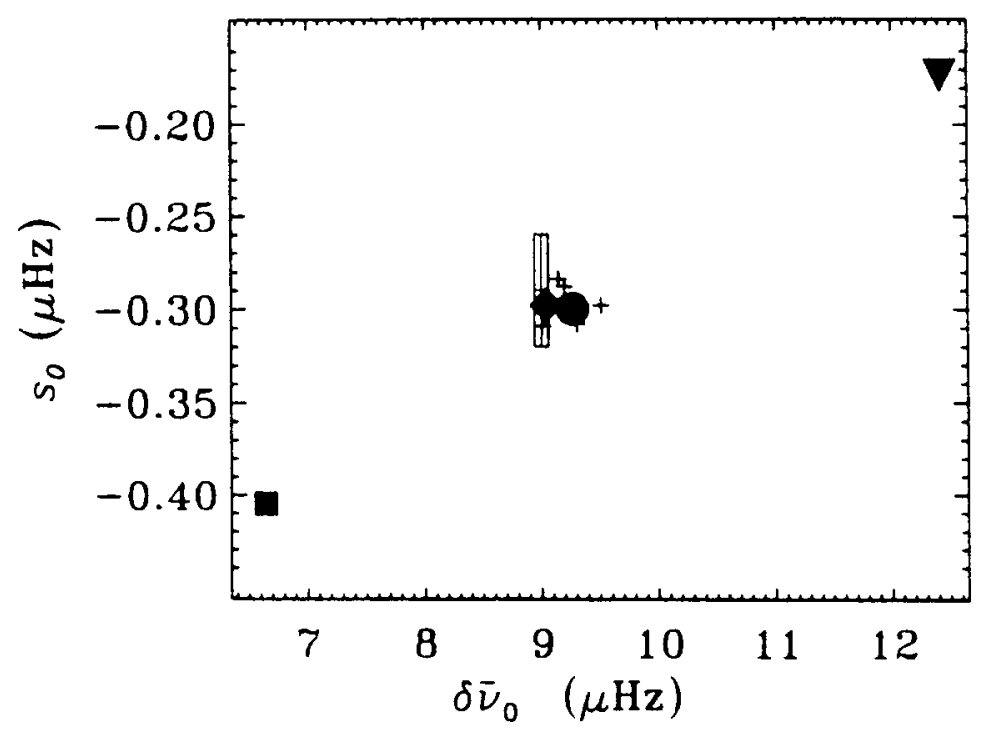

Figure 16: Parameters in least-square fit to the small frequency separation (cf. equation 66), using a reference order $n_{0}=21$. The small crosses show various normal solar models using varying physics. The filled circle and diamond are models with up-to-date physics, without and with helium settling, respectively. The filled square shows results for the model with reduced core opacity, illustrated in Figs 14 and 15, while the filled triangle is a model with a partially mixed core. The error box shows observed values from Elsworth et al. (1990).

As a result of this $l$-dependence the small frequency separation $\delta_{n l}$ ( $c f$. equation 38 ) is reduced substantially by the reduction in the core opacity. To illustrate the effect, and compare it with unmodified solar models and the observed values, I follow Elsworth et al. (1990), approximating $\delta_{n l}$ as

$$
\delta_{n l} \simeq \bar{\delta}_{l}+s_{l}\left(n-n_{0}\right),
$$

where $n_{0}$ is a suitable reference order, and the coefficients $\bar{\delta}_{l}$ and $s_{l}$ are determined through a least-squares fit. The results are shown in Figure 16. Normal solar models, particularly with the inclusion of helium settling in the core, are in good agreement with the observations. In contrast, the model with reduced core opacity is clearly inconsistent with the observed values. The figure also shows results for a model with partial mixing of the core, based on the hydrogen profile of Schatzman et al. (1981). This has a reduced neutrino flux relative to 
normal models, but is again inconsistent with the observed frequency separation, although the computed value is now too large.

Results such as these clearly argue against an astrophysical solution to the solar neutrino problem: there is a strong tendency that proposed models with reduced neutrino fluxes are inconsistent with the helioseismic data. However, as mentioned in Section 3.2 the observed frequencies do not in themselves constrain the solar internal temperalure and hence the neutrino flux. In fact, one might inagine constructing a model involving hoth reduced core opacity and partial mixing, choosing the magnitude of the effects such as to bring both oscillation frequencies and neutrino fluxes into agreement with the measured values. Such a model would clearly be somewhat contrived. and hardly plausible; but its possible existence highlights the need for further assumptions, if helioseismology is to provide constraints on the neutrino production of the solar core.

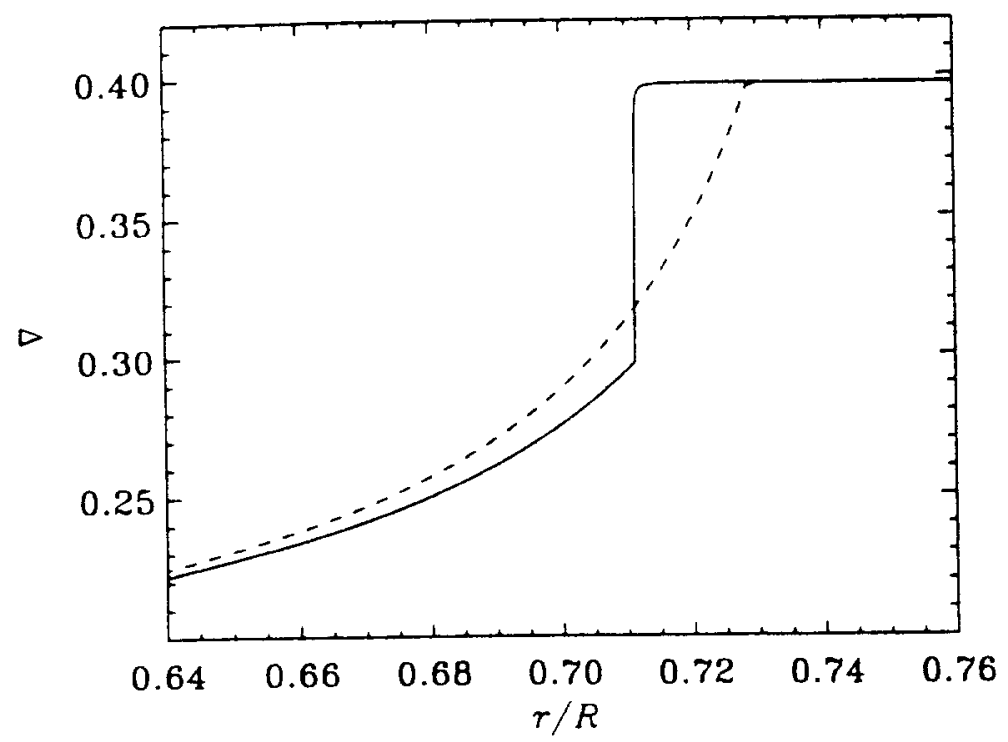

Figure 17: Temperature gradient $\nabla=\mathrm{d} \ln T / \mathrm{d} \ln p$ in normal solar model (dashed line) and model with overshoot of $0.21 H_{p}, H_{p}$ bcing the pressure scale height. (Adapted from Monteiro et al. 1994.)

\subsection{The base of the convection zone}

Normal solar models predict a sharp transition between the lower part of the convection zone, where the temperature gradient is very nearly adiabatic, and the radiative region below where the temperature gradient decreases sharply with the increasing temperature. This is illustrated in Figure 17. It is evident 
that the sound-speed gradient displays a very similar behaviour, resulting in a near-discontinuity in the second derivative of sound speed at the base of the convection zone. The resulting inflection was visible even in early determinations of the solar sound speed through asymptotic inversions of observed frequencies (Christensen-Dalsgaard et al. 1985). Christensen-Dalsgaard, Gough \& Thompson (1991) carried out careful analyses to determine the location of the break in the gradient of the sound speed as inferred from inversion, and testing the methods on artificial data; in this way they determined the depth of the solar convection zone as $d_{\mathrm{b}}=(0.287 \pm 0.003) R$.

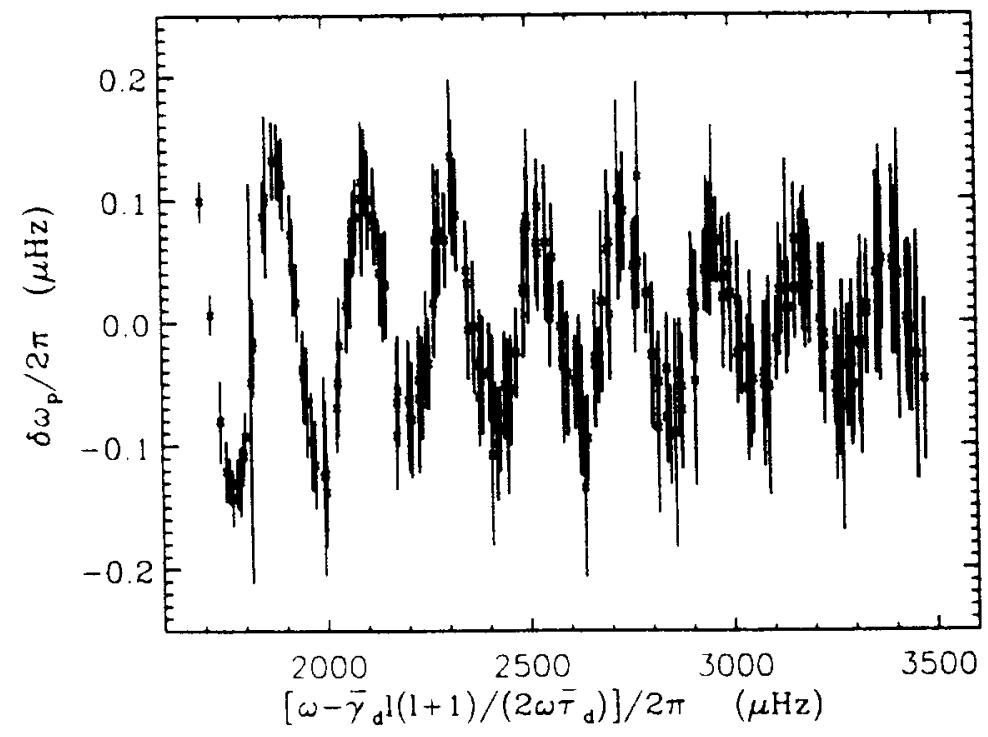

Figure 18: Oscillatory signal corresponding to the base of the convection zone, for observed solar frequencies from Libbrecht, Woodard \& Kaufman (1990), plotted against reduced frequency. The parameters $\bar{\gamma}_{\mathrm{d}}$ and $\bar{\tau}_{\mathrm{d}}$ (the latter being the acoustical depth of the discontinuity) have been obtained from a fit to the data. (From Monteiro et al. 1994.)

The discontinuity in the second derivative is a sharp feature, in the sense introduced in Section 3.4.3, and hence may be expected to introduce an oscillatory signal in the oscillation frequencies. An even more extreme behaviour is predicted by simple models of convective overshoot ( $c f$. Section 2.3.1). The resulting temperature gradient, also illustrated in Figure 17, is essentially discontinuous at the point where the motion stops. Thus at this point there is also a discontinuity in the sound-speed gradient, which again introduces oscillations in the frequencies.

The detailed effects on the oscillation frequencies of these properties were analyzed by Basu, Antia \& Narasimha (1994), Monteiro et al. (1994), Roxburgh 


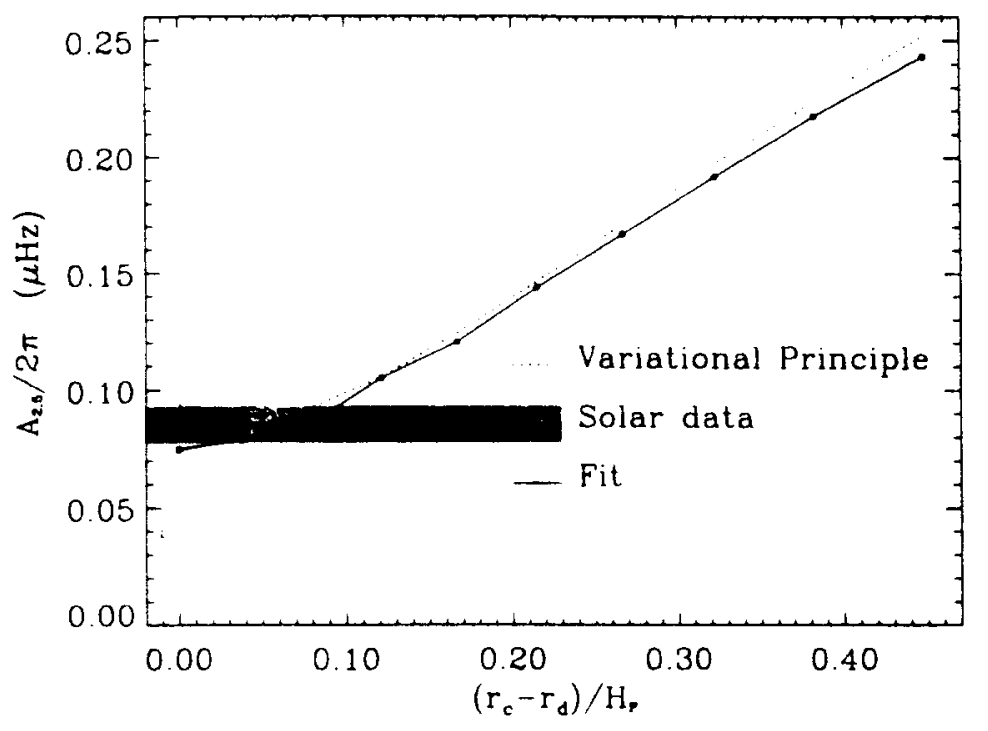

Figure 19: Normalized amplitude of the oscillatory frequency signal induced by the base of the convection zone, as a function of overshoot distance in units of the pressure scalf height $H_{p}$. The solid line shows a fit to computed frequencies, whereas the dotted line was obtained from an analytical approximation to the amplitude. The shaded arca shows the amplitude inferred from the solar data (Libbrecht el al. 1990), with a width corresponding to an estimate of its error. (From Christensen-Dalsgaard et al. 1995a.)

\& Vorontsov (1994) and Christensen-Dalsgaard, Monteiro \& Thompson (1995a). As shown by Monteiro et al. (1994) the signal in the frequencies is essentially a function of $\omega-\gamma_{\mathrm{d}} L^{2} /\left(2 \omega \tau_{\mathrm{d}}\right)$, where $\tau_{\mathrm{d}}$ is the acoustical depth of the discontinuity (in the first or second sound-speed derivative) and $\gamma_{\mathrm{d}}=\int_{r_{\mathrm{d}}}^{R}\left(c / r^{2}\right) \mathrm{d} r, r_{\mathrm{d}}$ being the radius of the discontinuity. In Figure 18 the oscillatory component of the observed frequencies have been plotted in this form. There is indeed a clear signal, with a 'frequency' which corresponds to the depth of the convection zone as inferred by Christensen-Dalsgaard et al. (1991).

Frequencies of solar models without and with overshoot show a similar behaviour. However, the amplitude of the oscillatory signal depends on the extent of overshoot. Figure 19 shows fitted amplitudes, normalized to a frequency of $2.5 \mathrm{mHz}$, as a function of the overshoot distance in units of the pressure scale height $H_{p}$, together with the similarly fitted amplitude for the solar frequencies. It is evident that in the Sun overshoot of this form can at most extend approximately $0.1 H_{p}$ (Basu et al. 1994; Monteiro et al. 1994).

Although these results place interesting constraints on the structure at the 
base of the solar convection zone, it must be realized that the proposed model for overshoot is highly simplified. In particular, it assumes that the effects on the frequencies can be characterized by a spherically symmetric and time-independent structure. In reality, overshoot must display substantial variations as a function of position and time. The oscillations sense an average of these variations; thus it is no surprise that the observed frequencies indicate a relatively smooth structure. More careful investigations, involving also hydrodynamical simulations of conditions at the base of the convection zone, will be required to obtain firmer helioseismic bounds on the extent of overshoot.

\subsection{The equation of state}

It was argued in Section 2.4 that the convection zone is well suited for studies of the properties of the equation of state of solar matter. Although conditions very near the surface are complicated by the uncertain structure of the strongly superadiabatic region, in the deeper parts of the convection zone the stratification is very nearly adiabatic, and the structure is largely controlled by the equation of state and the composition.

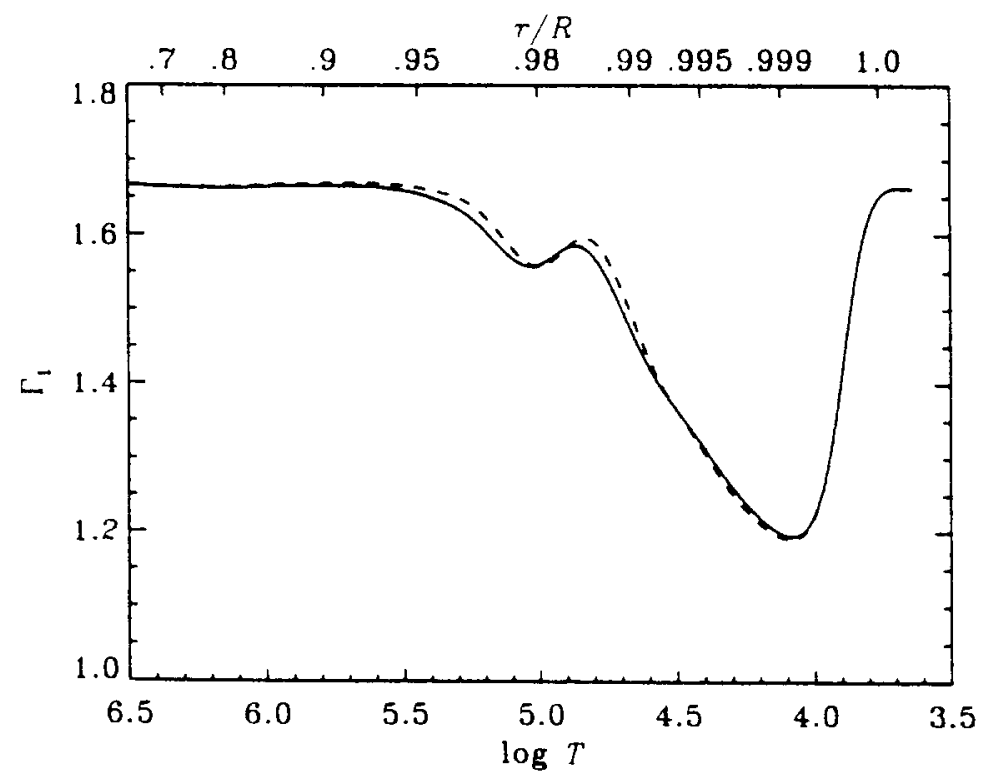

Figure 20: Adiabatic exponent $\Gamma_{1}=(\partial \ln p / \partial \ln \rho)_{s}$, plotted at the conditions $(\ln \rho, \ln T)$ in a normal solar model. The lower abscissa indicates $\log T(\log$ being to base 10) while the upper abscissa shows the corresponding fractional radius $r / R$. The solid line was obtained using the EFF equation of state, while the dashed line is based on the CEFF formulation.

Much of the uncertainty in the treatment of the thermodynamic properties 
of solar matter is related to the innization processes, which depend crucially on the interactions between the constituents of the gas. The hydrogen and first hclium ionization zones are situated so close to the surface that their structure may be affected by the uncertain physics of convection. However, at the second helium ionization zone these offects are probably sufficiently weak that analysis of the influence of this region on the frequencies may be used for tests of the equation of state, or for determinations of the solar envelope helium abundance.

In the present section I illustrate the sensitivity of the structure and the oscillation frequencies to the treatment of the thermodynamic properties, by considering formulations of increasing complexity [see Christensen-Dalsgaard \& Däppen (1992) for a much more detailed treatment]. Comparisons with the observed frequencies are deferred to Section 5.2 .

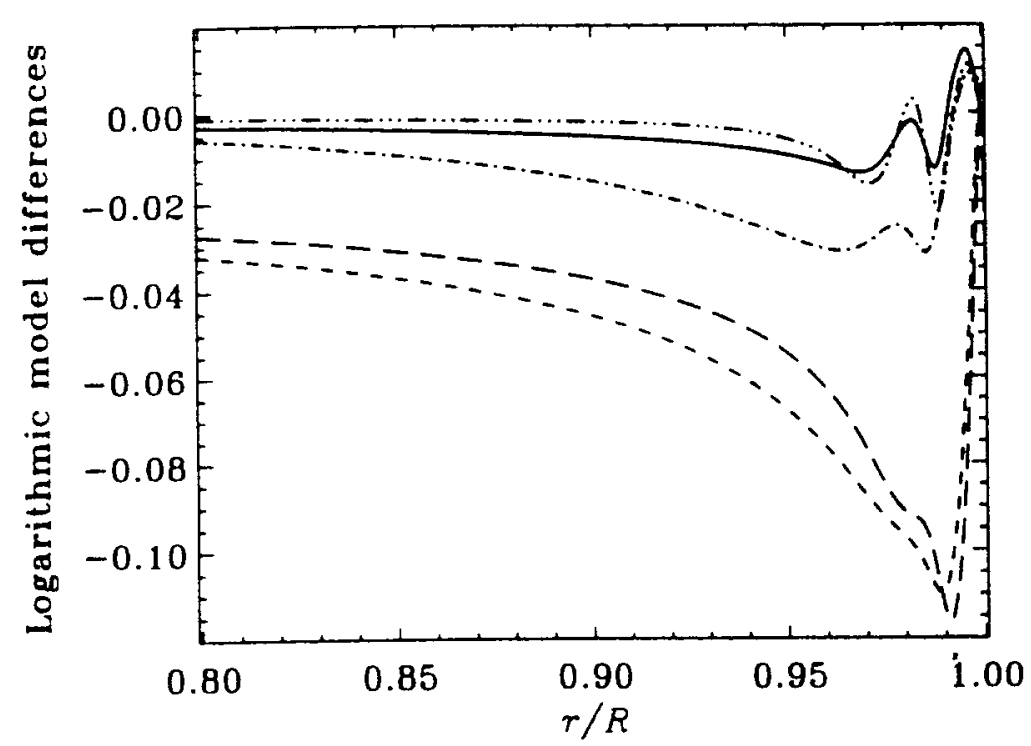

Figure 21: Logarithmic differences at fixed radius $r$ between models computed with the EFF and CEFF equations of state, in the sense (EFF) - (CEFF). The following line styles have been used: $\delta \ln c:-; \delta \ln p: \ldots . . . . . .$. $\delta \ln \rho:-\cdots---; \delta \ln T:-\cdot-\cdots ; \delta \ln \Gamma_{1}:-\cdots-\cdots-\cdots$.

\subsubsection{Comparison of EFF and CEFF formulations}

The EFF and CEFF treatments (see Section 2.2.1 for details) differ in the inclusion in CEFF of Coulomb effects. These predominantly affect the structure of the convection zone through a change in the ionization balance. To illustrate this, Figure 20 shows $\Gamma_{1}$ computed with the EFF and CEFF equations of state, 
at conditions corresponding to a solar model. 'The ionization zones are reflected by dips in $\Gamma_{1}$ : the dominant dip near the surface results from the combined effects of the hydrogen and first helium ionization zones, while the second helium ionization zone is visible as a separate dip, at $r \simeq 0.98 R, \log T \simeq 5$. Inclusion of Coulomb effects, illustrated by the dashed line, causes a shift of the second helium zone towards lower temperature.

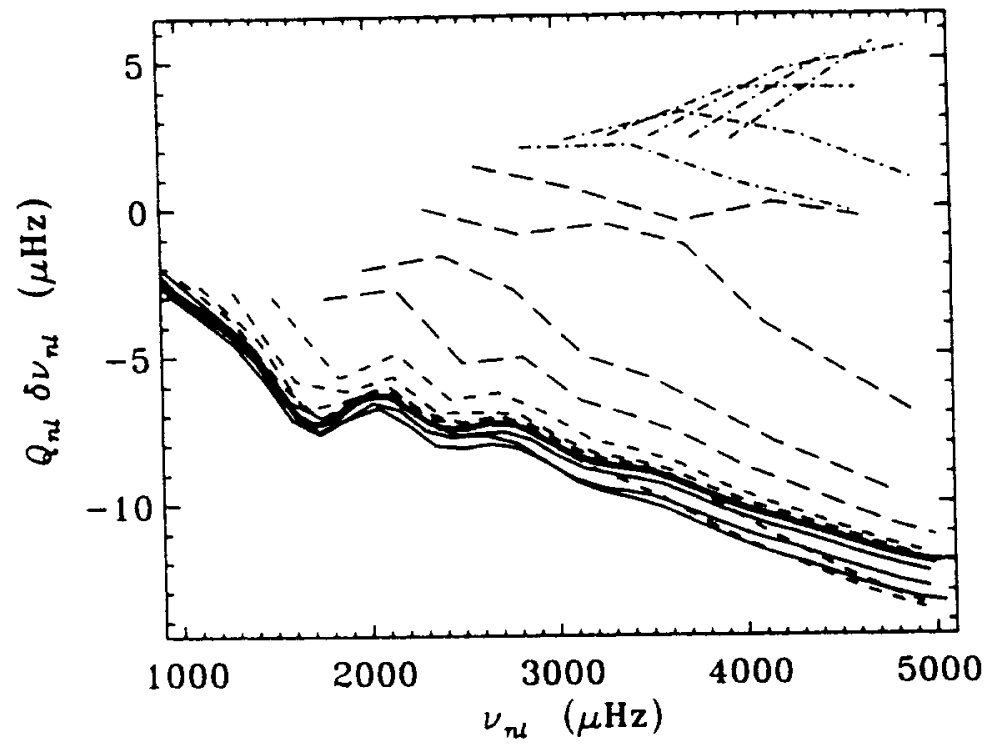

Figure 22: Scaled frequency differences corresponding to the differences illustrated in Figure 21 between models computed with the EFF and CEFF equations of state. Modes of the same degree l have been connected, according to the following line styles: $l=0-30:-; l=40-100: \ldots \ldots \ldots \ldots, l=150-400$ : $\ldots+\ldots ; l=500-1100: \ldots . \cdots$.

Differences between models computed with the EFF and CEFF equations of state are illustrated in Figure 21. The shift in the ionization causes an oscillatory feature in $\delta \Gamma_{1}$ which is reflected in the sound-speed difference. The effects in the inner parts of the model are considerably smaller. The corresponding scaled frequency differences are shown in Figure 22. These are dominated by the model changes in the hydrogen and helium ionization zones, leading the frequency differences which depend little on degree for $l \$ 100$; here the variation with frequency clearly displays an oscillation associated with the second helium ionization zone. For higher degree, the modes get trapped close to the surface and hence are affected predominantly by the positive sound-speed difference in the hydrogen and first helium ionization zones. It should be noticed that the frequency changes are comparatively large, up to about $10 \mu \mathrm{Hz}$. Hence they are easily visible in the observed frequencies. Indeed, it was shown by Christensen-Dalsgaard, Däppen \& Lebreton (1988) that the observations were 

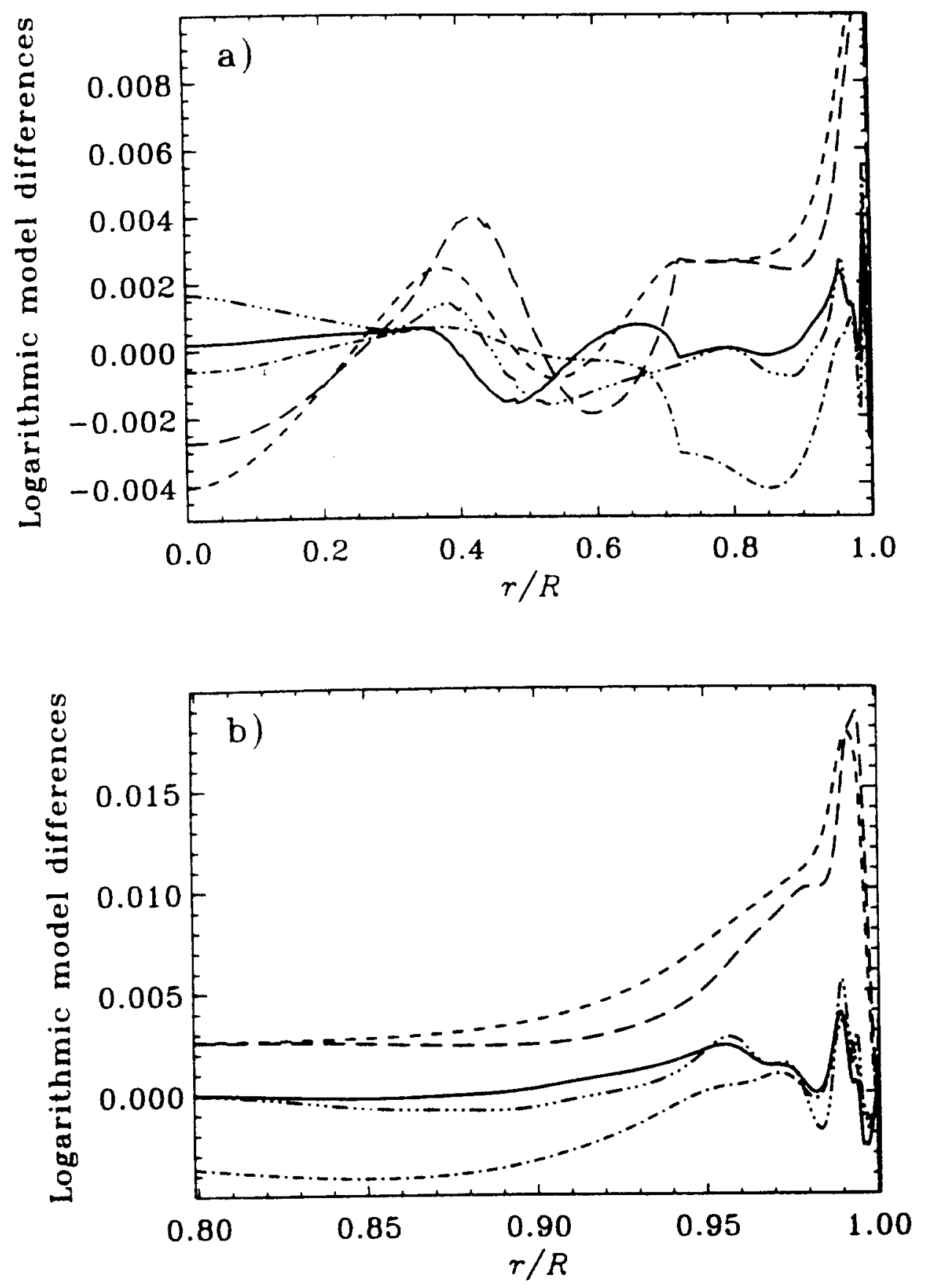

Figure 23: Logarithmic differences at fixed radius $r$ between models computed with the CEFF and MHD equations of state, in the sense (CEFF) - (MHD). The following line styles have been used: $\delta \ln c:-; \delta \ln p: \ldots . . . . . . .$. $\delta \ln \rho:--\ldots--; \delta \ln T:--\cdots-\ldots ; \delta \ln \Gamma_{1}:-\cdots-\cdots-\cdots$. Panel (a) shows the entire model, panel (b) the outer parts of it. 

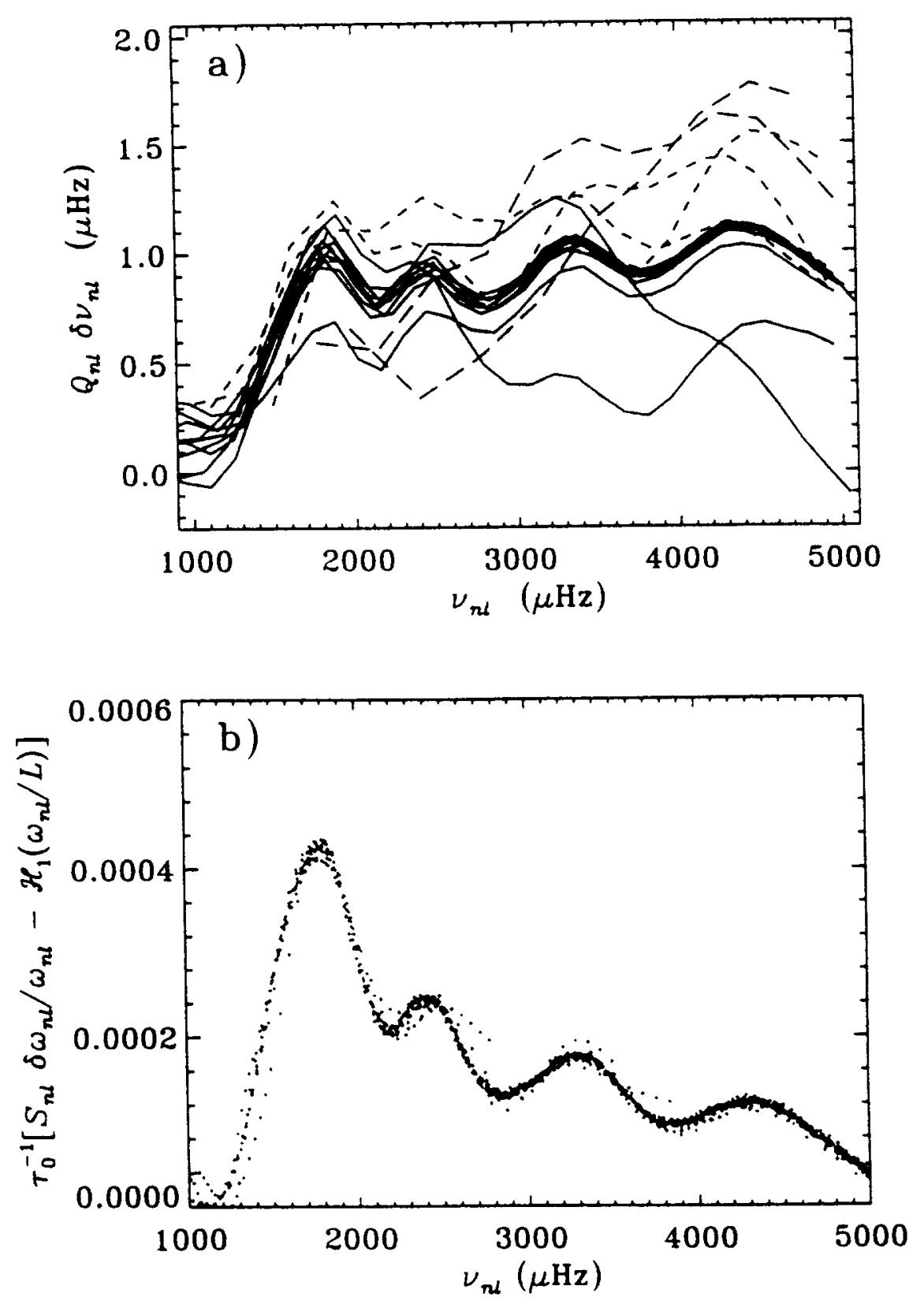

Figure 24: Frequency differences corresponding to the differences illustrated in Figure 23 between models computed with the CEFF and MHD equations of state. (a) Original scaled frequency differences. Modes of the same degree l have been connected, according to the following line styles: $l=0-30:-; l=$

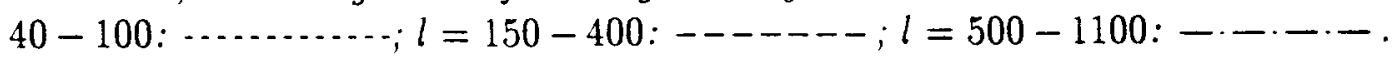
(b) Scaled differences after subtraction of the function $\mathcal{H}_{1}(\omega / L)$ resulting from the spline fit in equation (23). 
(dearly inconsistent with the KFF equation of state (see also Section 5.2 below).

\subsubsection{Comparison of CEFF and MHD formulations}

To illustrate the considerable sensitivity of the oscillation frequencies to the ecpuation of state I next consider differences between models computed with the CEFF and MHD expuations of state. These formulations differ mainly in the treatment of the partition function. The resulting model differences, shown in Figure 2:3, display a great deal of structure, much of it evidently still associated with the hydrogen and helium ionization zones. Also, the changes are roughly an order of magnitude smaller than those obtained between the EFF and CEFF models. The associated scaled frequency differences, shown in Figure 24(a), are correspondingly smaller and somewhat more complex than those obtained for the $\mathrm{EFF}$. ( $\mathrm{EFF}$ differences. Even so, the oscillatory signature of the second helium ionization zone is clearly visible. Also, it should be noticed that the magnitude of the differences is still much bigger than the observational error; thus it is plausible that the observations can distinguish between these two formulations. In Section 5.2 I show that this indeed the case.

The finer details in the frequency differences can be shown more clearly by carrying out a fit of the form given in equation (23). Here I concentrate on the function $\mathcal{H}_{2}(\omega)$, by showing in Figure $24(b)$ the residuals after subtraction of the fitted function $\mathcal{H}_{1}(\omega / L)$. These residuals are indeed predominantly a function of frequency and very clcarly displays an oscillation, with a 'period' approximately corresponding to a feature at the location of the second helium ionization zone.

\section{ANALYSIS OF OBSERVED FREQUEN- CIES}

In the preceding section I considered a few aspects of the observed frequencies of solar oscillation; these indicated that the structure of the solar core is similar to that of normal solar models, and placed stringent limits of a possible adiabatic extension of the convective envelope through convective overshoot. Here I make a more detailed comparison of the observed frequencies with the models.

Two different sets of observed frequencies are used in the analysis. One is the compilation by Libbrecht et al. (1990) which combines Big Bear Solar Observatory data for $l \geq 3$ with low-degree data obtained from whole-disk observations (Jiménez et al. 1988). In the second (in the following BISON-BBSO) 


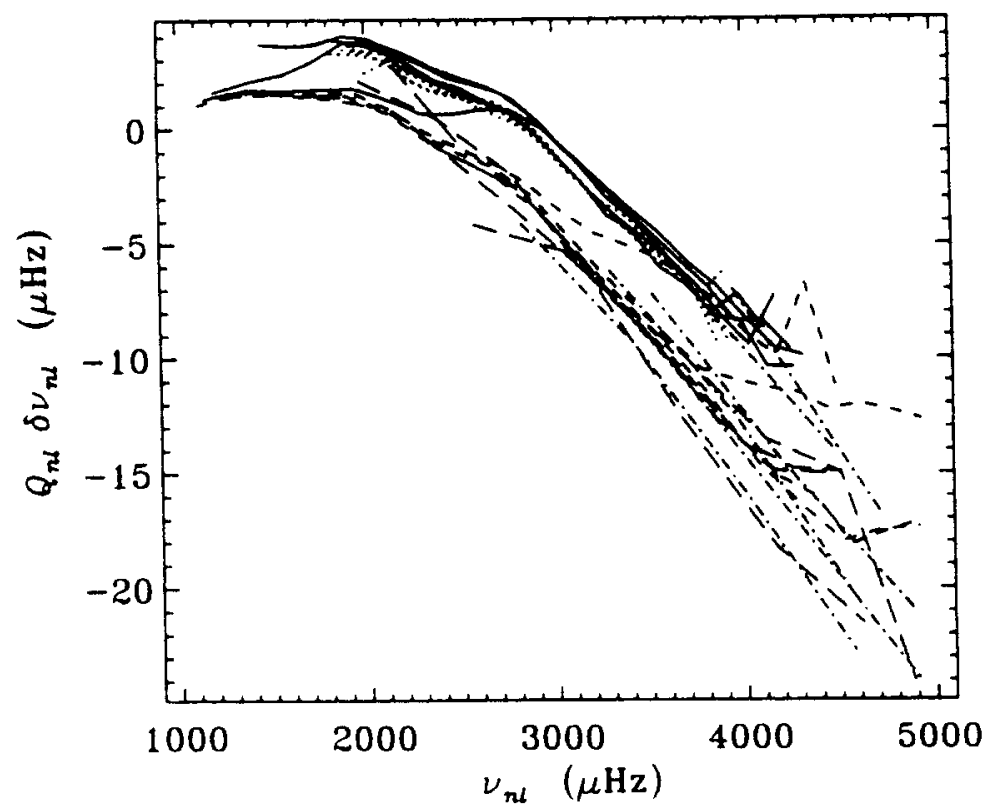

Figure 25: Scaled frequency differences between the BISON-BBSO set of observed frequencies and the normal solar model of Christensen-Dalsgaard et al. (1993), in the sense (observations) - (model), plotted against frequency. Modes of the same degree $($ have been connected, according to the following line styles: $l=0-$ $3: \cdots \ldots \ldots \ldots \ldots, l=4-30: \longrightarrow ; l=40-100 ; \ldots \ldots, \ldots, \cdot l=150-400$ : $------; l=500-1100:-\cdots \cdot-\ldots$.

the modes with $l \leq 3$ were obtained from more recent data from the BISON network (Elsworth et al. 1994). The observations are compared with models differing predominantly in the equation of state, or in the inclusion or eglect of diffusion and gravitational settling of helium. Except where otherwise noted, the models were computed as done by Christensen-Dalsgaard et al. (1993). This includes the CEFF equation of state, OPAL opacities and the Parker (1986) nuclear reaction parameters. Calibration to the luminosity of the present Sun required an initial helium abundance $Y_{0} \simeq 0.28$.

\subsection{Asymptotic analysis of observed frequencies}

A first step in the analysis is evidently to consider differences between observed and computed frequencies. The suspicion of problems in the superficial layers of the models motivates the inclusion of scaling by the normalized mode inertia $Q_{n l}$ or the equivalent $S_{n l} / \tau_{0}(c f$. Section 3.4.4). Typical results, for a normal solar model with no diffusion, are shown in Figure 25. It is evident that the 
scaled differences do indeed depend mostly on frequency, as would be the case if the errors in the model were concentrated near the surface. The departures from this trend are so small as to be barely visible in a plot of this form. The dominant effect is a separation between modes of degree $l \leq 20$ and $l \geq 50$, with a Iransition for intermediate modes. This is strongly reminiscent of the hehaviour found in Sections 4.2 and 4.3 to result from an increased depth of the convection zone, suggesting that the convection zone in the model is too shallow (this effect was first noticed by Christensen-Dalsgaard \& Gough 1984). Nonetheless, it is clear that a more careful analysis is required to uncover the details of this and possible other problems in the interior of the model.

To isolate the more subile features I employ the asymptotic analysis already used on computed differences in Section 4.3. Figure 26(a) shows the scaled differences (using now the asymptotic scaling) against $\nu / L$ and hence turningpoint position. It is evident already from this raw difference plot that in this case the term in $\mathcal{H}_{2}$ dominates, as was also noted in Figure 25. However, there is also evidence for a contribution from $\mathcal{H}_{1}$. This becomes clearer if the spline fit is arried out and the contribution from $\mathcal{H}_{2}$ is subtracted from the scaled differences. The result is shown in Figure 26(b), together with the fitted function $\mathcal{H}_{1}(\omega / L)$. There is again a sharp step corresponding in position to $r_{\mathrm{t}} \simeq 0.7 R$, i.c., the base of the convection zone. This confirms the evidence from the simple inspection of frequency differences in Figure 25 that the convection zone in the Sun is slightly deeper than in the model. Indeed, the convection-zone depth in the model is $d_{\mathrm{b}}=0.278 R$, somewhat smaller than the solar value of $d_{\mathrm{b}}=0.287 R$ inferred by Christensen-Dalsgaard et al. (1991).

It is evident that there is considerably more scatter in Figure 26(b) than in the corresponding Figure $12(b)$. This is due to observational errors, both random and systematic. In particular, it may be noticed that there is an apparent break at around $\nu / L \simeq 15 \mu \mathrm{Hz}$. In fact, the observed frequencies were obtained from two separate sets of observations, the merge taking place at $l=400$; it has later been found that there were slight systematic errors in the high-degree set. Furthermore, there appear to be problems at low degree, corresponding to the highest values of $\nu / L$. These difficulties are clearly reflected in the fitted $\mathcal{H}_{1}(\omega / L)$.

The residual after subtraction of the fitted $\mathcal{H}_{1}$ from the scaled differences, and the fitted $\mathcal{H}_{2}$, are shown in Figure 27. The residuals are indeed largely a function of frequency. They are dominated by a slowly varying trend which, as argued in Section 5.3 below, reflects errors in the near-surface region of the model. However, there is also a weak but clearly noticeable oscillatory signal. As discussed in Section 5.2 this probably reflects differences between the Sun and the model in the helium abundance and equation of state in the convective envelope. 

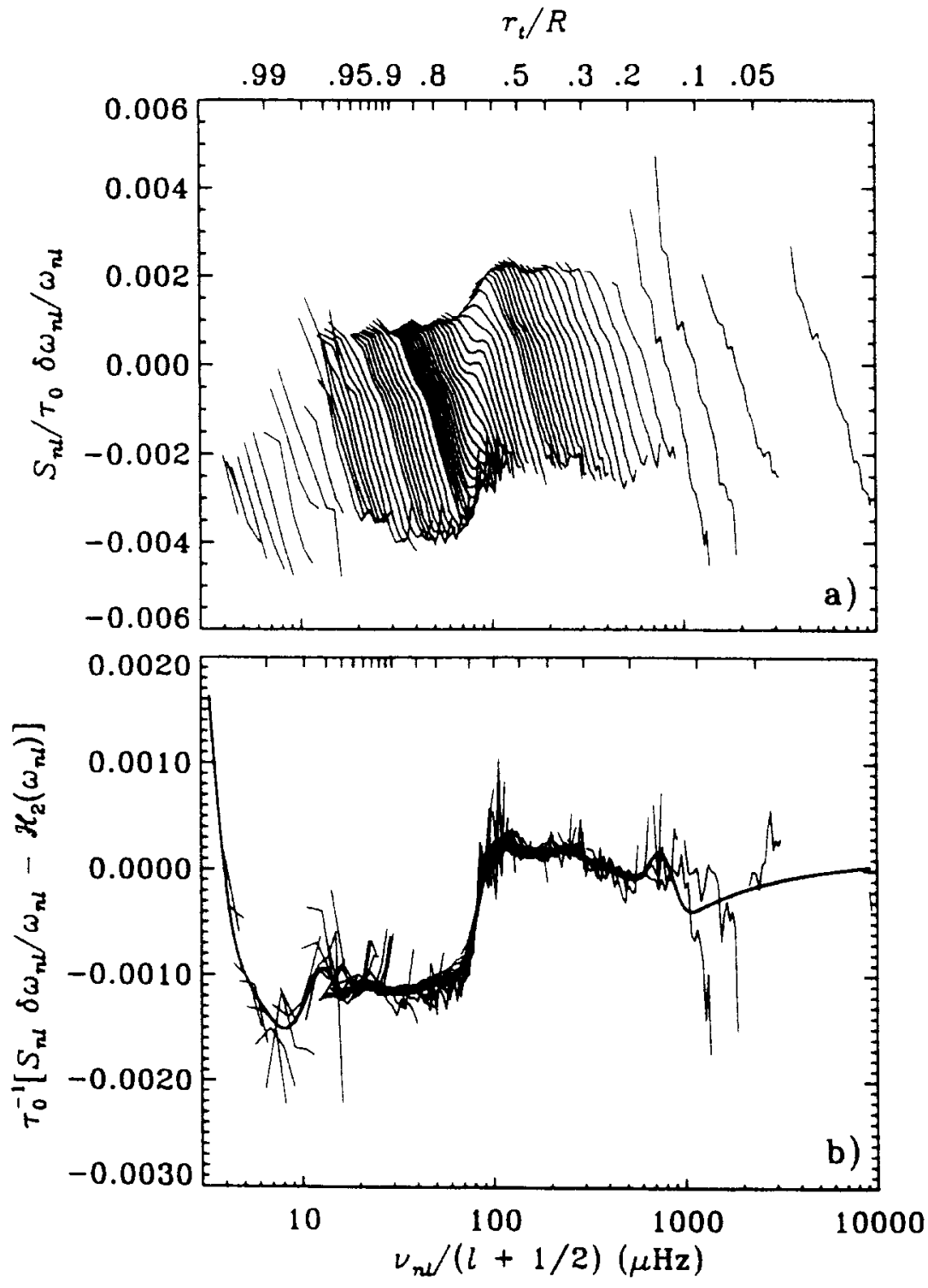

Figure 26: Asymptotically scaled frequency differences between the observed frequencies of Libbrecht et al. (1990) and the normal solar model of ChristensenDalsgaard et al. (1993), in the sense (observations) - (model), plotted against $\nu /(l+1 / 2)$. The upper abscissa shows the location of the lower turning point, which is related to $\nu /(l+1 / 2)$ through equation (10). (a) Original scaled frequency differences. (b) Scaled differences, after subtraction of the function $\mathcal{H}_{2}(\omega)$ obtained from the spline fit. The heavy solid line shows the fitted function $\mathcal{H}_{1}(\omega / L)$. 


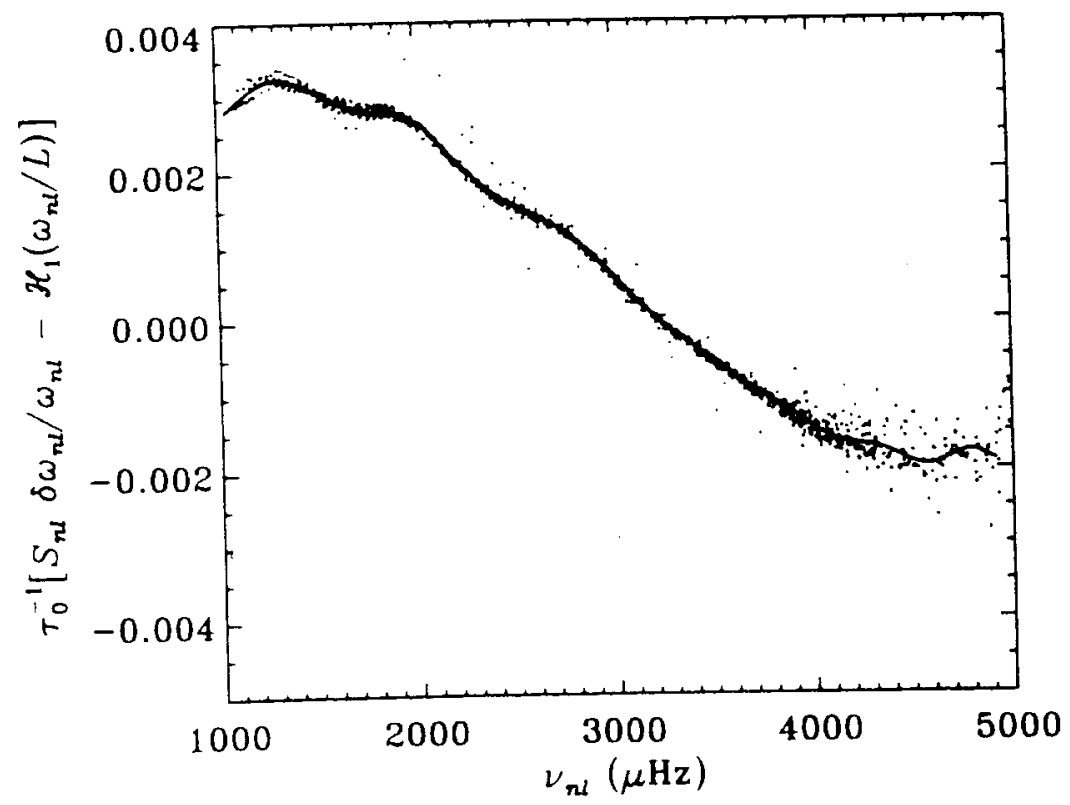

ligure 27: Scalcd frequency differences between observations and model, shown in Figure $20(\mathrm{a})$. after subtraction of the function $\mathcal{H}_{1}(\omega / L)$ resulting from the spline fit. The heary solid line shows the fitted function $\mathcal{H}_{2}(\omega)$.

The function $\mathcal{H}_{1}(\omega / L)$ is related to the sound-speed difference between two models, or between the Sun and a model, through equation (24). Given a determination of $\mathcal{H}_{1}$ this equation is an integral equation for $\delta c / c$, with the solution

$$
\frac{\delta c}{c}=-\frac{2 a}{\pi} \frac{\mathrm{d}}{\mathrm{d} \ln r} \int_{a_{s}}^{a}\left(a^{2}-w^{2}\right)^{-1 / 2} \mathcal{H}_{1}(w) \mathrm{d} u
$$

(Christensen-Dalsgaard th al. 1989), where $a=c / r$ and $a_{\mathrm{s}}=a(R)$. This provides one of the simplest examples of an inversc analysis to infer properties of the solar interior from the observed frequencies (see the chapter by Gough). In fact, the asymptotic relation (12) and refinements of it also lead to absolute inversion methods whereby the solar sound speed is determined without reference to a solar model ( $\epsilon . g$. Gough 1984b; Christensen-Dalsgaard et al. 1985; Vorontsov \& Shibahashi 1991).

Tests of the inversion method given by equation (67) show that it provides reasonably accurate results in the range $0.2 R<r<0.95 R$, where the asymptotic description is approximately valid (Christensen-Dalsgaard et al. 1989). Here I apply it to the differences between the solar and the computed frequencies shown in Figure 26. The results are shown as the dotted line in Figure 28. The thin lines illustrate the effects of the error estimates for the solar frequencies, as quoted by the observers. Evidently the formal error on the result is extremely 


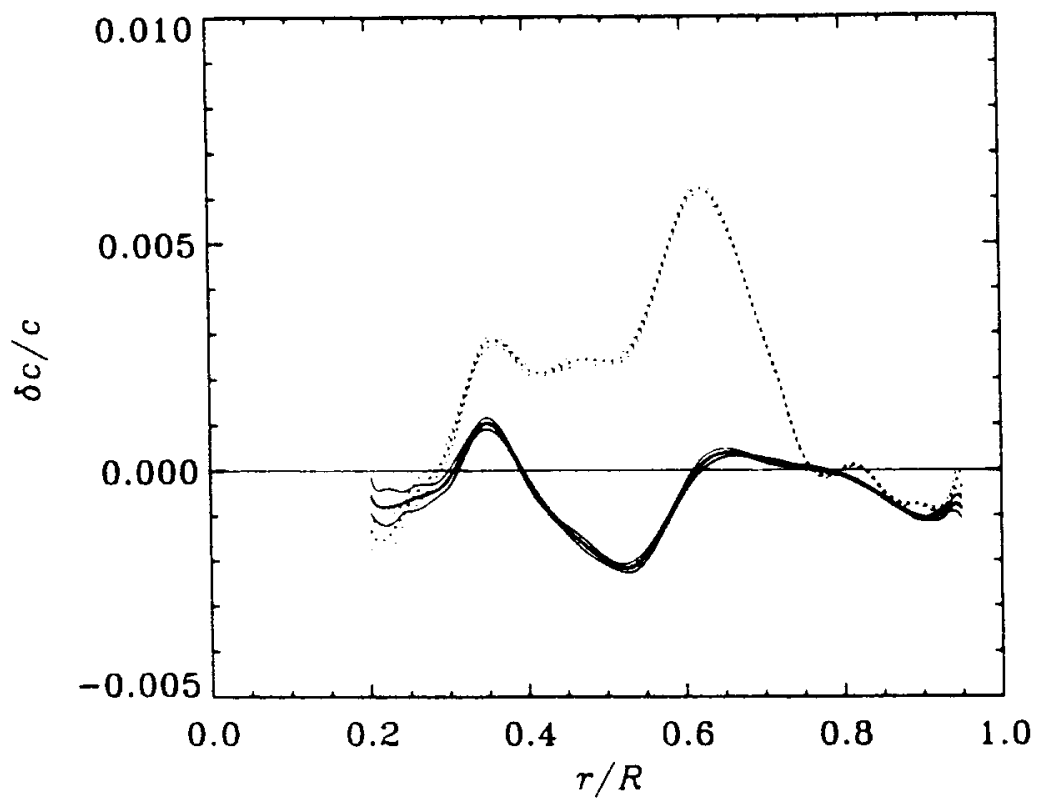

Figure 28: The dotted line shows the sound-speed difference $\delta c / c$ between the Sun and a normal solar model, inferred by applying equation (67) to the function $\mathcal{H}_{1}(\omega / L)$ shown in Figure $26(\mathrm{~b})$, corresponding to differences between observed and model frequencies. The solid line shows $\delta c / c$, similarly obtained, between the Sun and a model including diffusion and gravitational settling of helium. The thin lines indicate $1 \sigma$ error limits, based on the errors in the observed frequencies. Adapted from Christensen-Dalsgaard et al. (1993).

small. Also, the sound-speed differences are small, corresponding to errors in $T / \mu$ in the models of generally less than 1 per cent. Nonetheless, the differences are clearly much larger than the errors resulting from the observations.

To illustrate the effects of improvements in the description of the solar interior the solid lines show the sound-speed difference obtained from the differences between the solar frequencies and those of a model including the effects of helium diffusion and settling (Christensen-Dalsgaard et al. 1993). It is interesting that the relatively subtle, and previously commonly neglected, effect of gravitational settling leads to a substantial improvement between the model and the observations, partly by increasing the depth of the convection zone in the model. This is a striking illustration of the power of helioseismology to probe the details of the physics of the solar interior. Furthermore, it should be stressed that the models were computed without any attempt to match the observed frequencies. It is remarkable that our relatively simple description of solar evolution, using physics based on laboratory experiments, permits us to reproduce the sound speed in the solar interior to within a fraction of a per cent. On the other hand, 
it must be pointed out that the model is not unique: it is likely that modest modifications in the opacity. well within the precision of current opacity tables, might introduce changes in the sound speed of similar magnitude. The separation of opacity uncertainties from effects of diffusion and settling is a major challenge, which will undoubtedly require better physical understanding of the processes involved.

The results of Christensen-Dalsgaard et al. (1993) are in apparent conflict with those obtained by Ginenther, Pinsonneault \& Bahcall (1993), who concluded that current frequencies do not permit a definite helioseismic test of the effects of diffusion. However, (illenther et al. (1993) based their analysis on simple differences between observed and computed frequencies. Thus their results were clominated by the the effects of the near-surface uncertainties in the model, causing frequency differences of order $10 \mu \mathrm{Hz}$, which apparently masked the rather smaller effects resulting from diffusion. This provides a clear illustration of the need for careful analysis to isolate the sometimes quite subtle features of the solar interior in the observed frequencies.

\subsection{Test of the equation of state}

In Section 4.61 demonstrated the sensitivity of the computed frequencies to clescription of the thermodynamics of the solar interior. A closely related issue is the use of the observed frequencies to determine the helium abundance in the convection zone. Here I illustrate how comparisons with the observed frequencies may be used to test the equation of state.

An early indication of the power of the frequencies in this regard was obtained by Christensen-Dalsgaard, Däppen \& Lebreton (1988) who compared observed frequencies with frequencies computed with the EFF and MHD equations of state ( $c$. Section 2.2.1). Figure 29 shows corresponding results, although comparing instead the EFF and CEFF formulations. In both cases, the differences are dominated by a frequency-dependent trend, clearly resulting from errors in the near-surface layers. However, it is evident that in the EFF model there is a very considerable dependence of the differences on degree at given frequency, indicating a dependence on the turning-point position and hence a sign of errors in the interior of the model. It should be noticed that this spread is found even amongst modes of degree $l \geq 50$ trapped in the convection zone, where the equation of state is the dominant source of uncertainty. Also, the differences are comparatively large at low frequency, again indicating a component of the error in the model outside the superficial layers. In contrast, the spread with $l$ is much reduced in the CEFF model; also, much of it occurs for modes with turning points near the base of the convection zone (compare, for example, with 

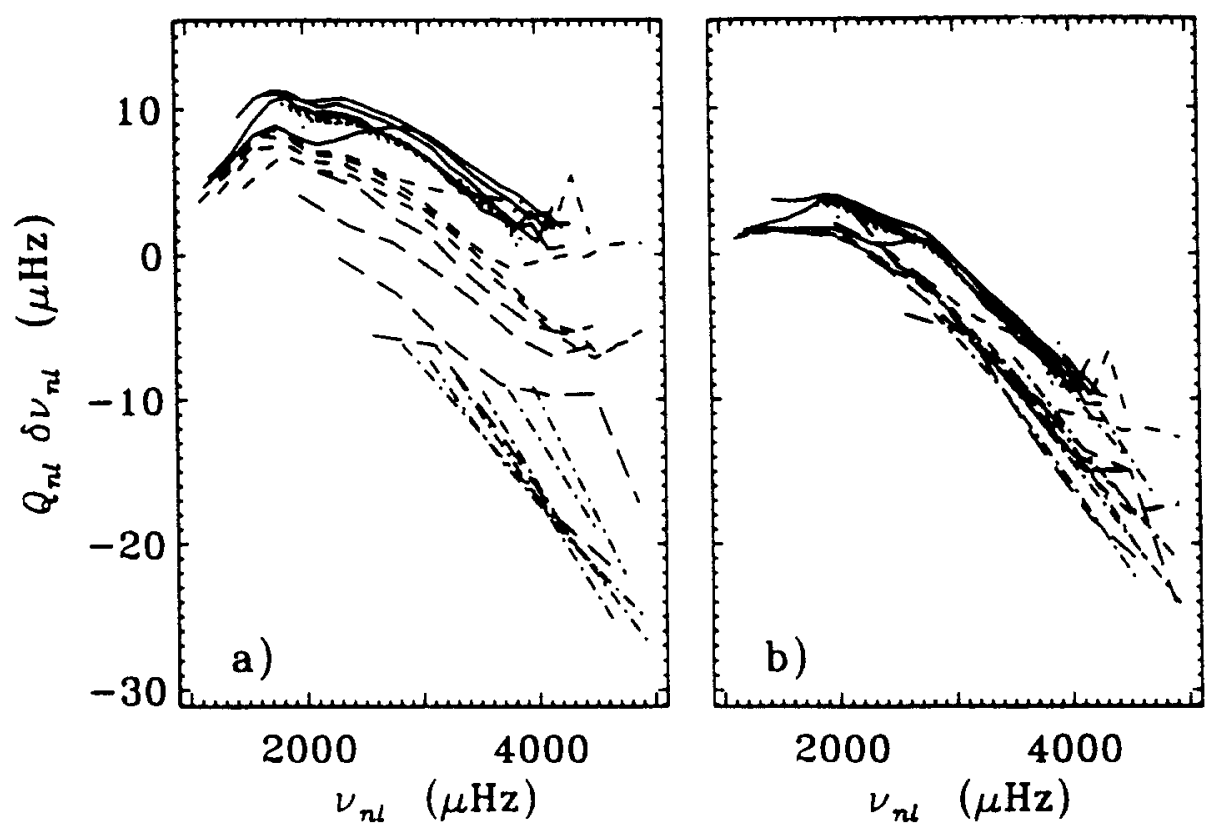

Figure 29: Scaled frequency differences between the BISON-BBSO set of observed frequencies and frequencies of two solar models, in the sense (observations) - (model), plotted against frequency. Modes of the same degree 1 have been connected, according to the following line styles: $l=0-3: \ldots \ldots \ldots \ldots \ldots$;

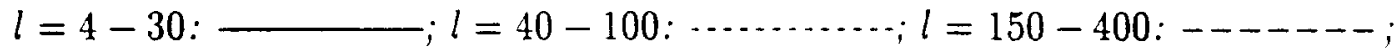
$l=500-1100:-.+-.+$. (a) Model computed with the EFF equation of state. (b) Model computed with the CEFF equation of state (this uses the same data as Figure 25).

Figure 10) and corresponds to the comparatively large sound-speed difference between the Sun and the model already inferred from the inversion in Section 5.1 ( $c f$. Figure 28). Furthermore, the differences at low frequency are now quite small. Thus there are strong indications that the CEFF model provides a better representation of the solar convection zone than does the EFF model. The improvement in the models resulting from the consistent inclusion of Coulomb effects was also noted by Stix \& Skaley (1990).

A more detailed comparison can be made, as usual, by carrying out a fit of the form given in equation (25) to the differences between the observed and computed frequencies. Figure 30 shows the resulting functions $\mathcal{H}_{1}(\omega / L)$ for models computed with the EFF, the CEFF and the MHD equations of state. The fit only determines $\mathcal{H}_{1}$ to within a constant: in fact, it follows from equation (67) that the sound-speed difference is essentially determined by the gradient of $\mathcal{H}_{1}$. Thus the figure clearly confirms the improved agreement resulting from using 


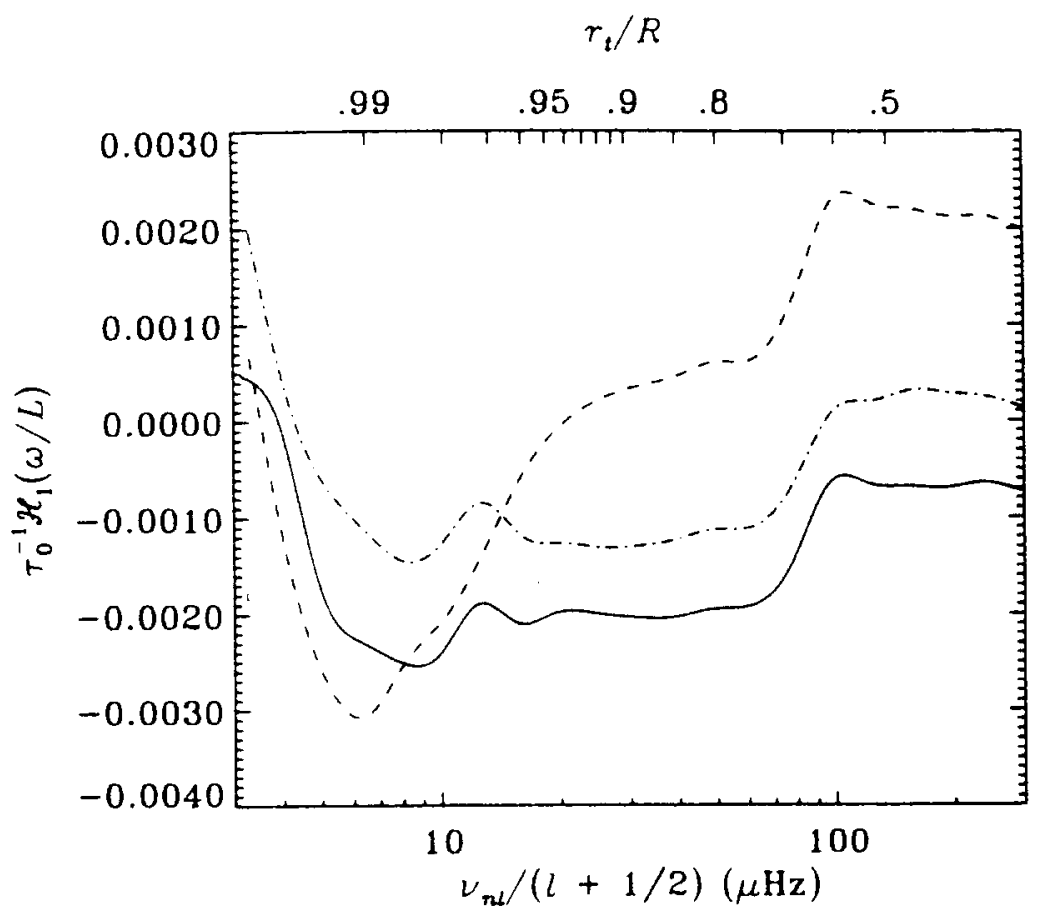

Figure 30: Functions $\mathcal{K}_{1}(\omega / L)$ fitted to asymptotically scaled frequency differinces between the BISON-BBSO set of observed frequencies, in the sense (observations) - (model), and three solar models: a model using the EFF equation of state (dashed line). a model using the CEFF equation of state (solid line), and a model using the MHD equation of state (dot-dashed line). The physics otheruise corresponds to the non-diffusive model of Christensen-Dalsgaard et al. (1993). The upper abscissa shows the location of the lower turning point, which is related to $\nu /(l+1 / 2)$ through equation (10).

the (EFF formulation, compared with EFF. On the other hand, there is no clear - distinction in this type of analysis between the MHD and CEFF formulations (see also Christensen-Dalsgaard \& Däppen 1992). The fairly steep rise in $\mathcal{H}_{1}(\omega)$ at low $\nu /(l+1 / 2)$ may be associated with near-surface errors in the model and oscillation physics or with errors in the assumed asymptotic formulation. Also, the curves clearly show the increase in $\mathcal{H}_{1}$ near $\nu /(l+1 / 2)=100 \mu \mathrm{Hz}$, associatcd with the difference in convection-zone depth between the models and the Sun. The effects of the equation of state on $\mathcal{H}_{1}(\omega / L)$ were also investigated by Antia \& Basu (1994) and Basu \& Antia (1995).

The phase function $\alpha(\omega)$ appearing in the Duvall law (equation 12), or the function $\mathcal{H}_{2}(\omega)$ obtained from the asymptotic fit, apparently provide even more sensitive discrimination between different equations of state and measures of the envelope helium abundance $Y_{\mathrm{e}}$. Vorontsov et al. (1991) analyzed properties of $\alpha(\omega)$ to infer that $Y_{e} \simeq 0.25$. A similar value was obtained by Christensen- 
Dalsgaard \& Pérez Hernández (1991) from analysis of $\mathcal{H}_{2}(\omega)$. Also, Pamyatnykh, Vorontsov \& Däppen (1991) investigated the sensitivity of functions related to $\alpha(\omega)$ to various aspects of the convective envelope. A serious problem in using these phase functions is the fact that they are generally dominated by contributions coming from the uncertain near-surface region. However, it was argued in Section 3.4.4 that these contributions are generally slowly varying functions of frequency, whereas contributions coming from somewhat deeper parts of the Sun oscillation with frequency (see also Figure 4 and Section 4.1 ). Vorontsov, Baturin $\&$ Pamyatnykh (1992) developed a polynomial fitting procedure which provided a separation between the slowly and the rapidly varying parts of the phase function. Working in terms of phase-function differences, Pérez Hernández \& Christensen-Dalsgaard (1994a) introduced a filtered function $\mathcal{H}_{2}^{\mathfrak{f}}(\omega)$, obtained by passing $\mathcal{H}_{2}(\omega)$ through a high-pass filter and hence suppressing the near-surface effects. Here I illustrate the use of such procedures by showing in Figure 31 the result of applying this filter to the $\mathcal{H}_{2}(\omega)$ resulting from differences between observed and computed frequencies, together with the original $\mathcal{H}_{2}(\omega)$. It is evident that the slowly-varying trend has indeed been eliminated, leaving an oscillatory function of frequency with a period corresponding roughly to the depth of the second helium ionization zone ( $c f$. Section 3.4.3).

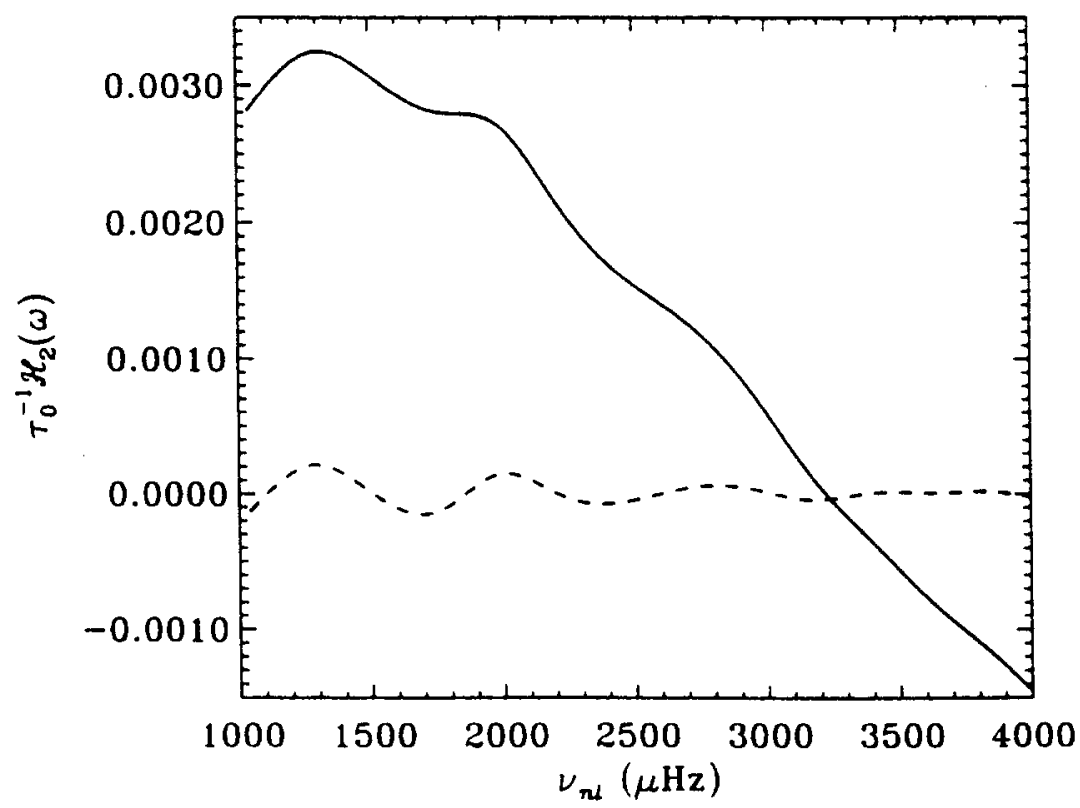

Figure 31: The solid curve shows the function $\mathcal{H}_{2}(\omega)$ obtained in a fit to scaled frequency differences between observations and model (see Figure 27). The dashed curve shows the result $\mathcal{H}_{2}^{\mathfrak{f}}(\omega)$ of applying the high-pass filter of Pérez Hernández \& Christensen-Dalsgaard (1994a) to this function.

The signal shown in Figure 31 contains contributions from several different 


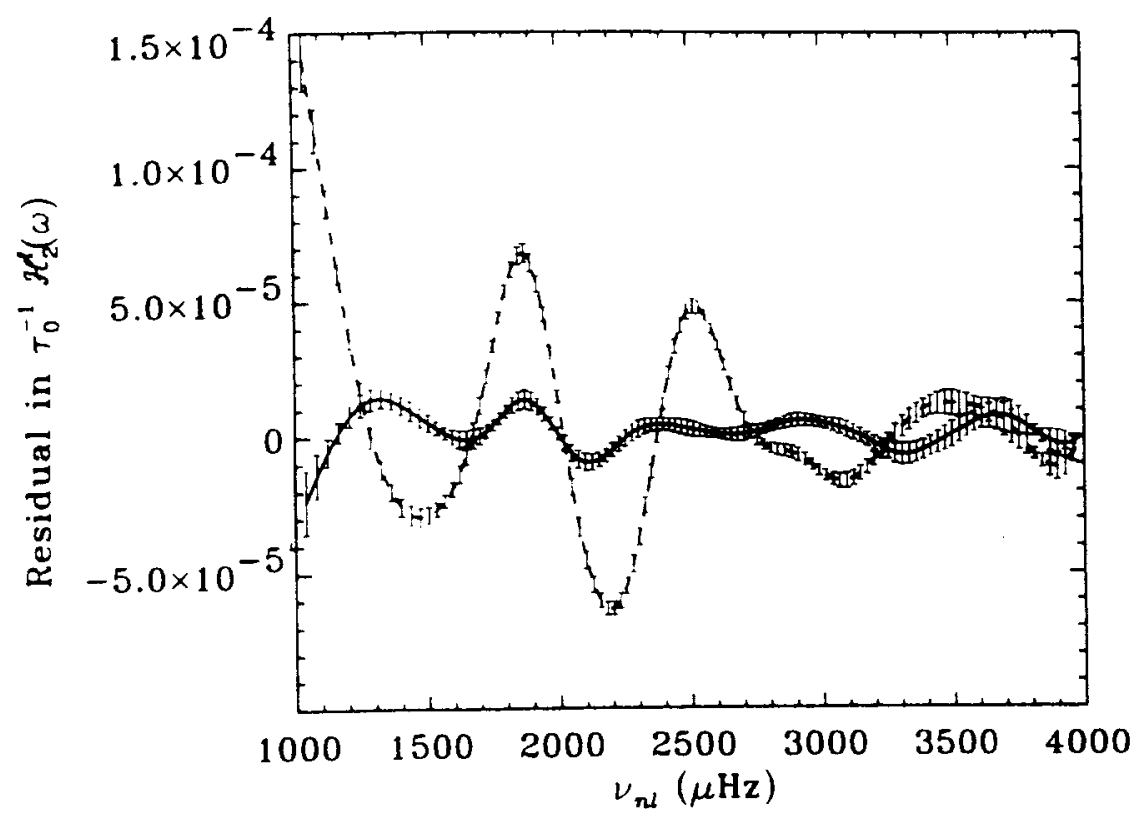

Figure 32: Rcsiduals, with 3o error bars, from fits of the filtered phase functions $\mathcal{H}_{2}^{\mathfrak{f}}(\omega)$ corresponding to differences between observed and model frequencies. The fits involve contributions from changes in the helium abundance, mixing-length parameter and near-surface structure. The solid curve corresponds to a reference model using the MHD tquation of state, while the dashed curve corresponds to the CEFF equation of state. (From Pérez Hernández \& Christensen-Dalsgaard 1994b.)

sources, including errors in the equation of state, differences between the composition and the specific entropy of the solar and the model convection zones and residual effects of the near-surface errors. To separate these effects Pérez Hernández \& Christensen-Dalsgaard (1994b) carried out a least-squares fit to the observed $\mathcal{H}_{2}^{f}(\omega)$ of a linear combination of three contributions: a contribution from a change in the envelope helium abundance; a contribution of a change in the mixing-length parameter (and hence the specific entropy in the adiabatic part of the convection zone); and a contribution corresponding to a change in the atmospheric opacity and representing effects of near-surface errors. The effect of each individual parameter was represented by a function $\mathcal{H}_{2}^{f}(\omega)$ obtained from differences between an envelope model incorporating a change in the given parameter and a reference model. Figure 32 shows the residuals of such fits to $\mathcal{H}_{2}^{f}(\omega)$ obtained from differences between the observed frequencies and frequencies for two reference models: a model computed using the CEFF equation of state and a model computed with the MHD equation of state. It is evident that the MHD model provides a much closer fit to the observations, when analyzed in this way, than does the CEFF model. In fact, the residual for the CEFF case 
is of the same order of magnitude as the oscillatory component of $\mathcal{H}_{2}(\omega)$ for the differences between the CEFF and MHD equations of state ( $c f$. Figure 24b); this suggests that there is relatively little cross-talk between the effects of using the CEFF equation of state and the remaining uncertainties in the model, in particular the helium abundance. It should be noted, however, than even for the MHD model the residuals are substantially larger than the $3 \sigma$ error bars shown in the figure, indicating that the MHD model is still not consistent with the Sun at the level of the observational errors. Nonetheless, these results suggest that the MHD equation of state provides a better representation of the thermodynamic properties of solar matter than the CEFF formulation, at least in the second helium ionization zone which dominates the signal shown in Figure 31. This is hardly surprising: the CEFF treatment is relatively simple, compared with the complex handling by MHD of interactions between the constituents of the gas.

A similarly detailed test of the Livermore equation of state ( $c f$. Section 2.2.1) has yet to be carried out. However, preliminary results indicate that the $\mathcal{H}_{2}(\omega)$ corresponding to the difference between models computed with the Livermore and the MHD equations of state differs in shape from the effect of a change in $Y_{e}$; this suggests that the Livermore formulation may be less successful in fitting the observations than MHD. On the other hand, it appears from sound-speed inversions there may be problems with the MHD formulation at temperatures somewhat exceeding that of the second helium ionization zone (Dziembowski et al. 1992).

As a result of their fit, Pérez Hernández \& Christensen-Dalsgaard (1994b) estimated the envelope helium abundance as $Y_{e} \simeq 0.242$, largely consistent with the value of 0.25 quoted above. Similar values were also obtained by Basu \& Antia (1995) from analyzing $\mathcal{H}_{1}$ and $\mathcal{H}_{2}$, using either the MHD or the Livermore equations of state. However, these results may still compromised by errors in the equation of state, and by possible systematic errors in the al alysis procedure. Kosovichev et al. (1992), using non-asymptotic inversion teciniques, made an extensive investigation of the uncertainties in the inferred value of $Y_{e}$ resulting from differences in the equation of state, the choice of mode set and the inversion procedure; the results suggest that current estimates of $Y_{e}$ must be viewed with some caution. Nevertheless, it is interesting to compare the results with those obtained from the calibration of solar models to the correct present luminosity, where a value of the initial helium abundance $Y_{0}$ of $0.27-0.28$ is typically required. This is probably inconsistent with the helioseismic estimates, even given the uncertainty in the equation of state. However, the results of Section 4.3 on models with helium diffusion and settling (see in particular Figure 11) shows that these effects reduce $Y_{e}$ by about 0.03 relative to the initial value. Thus for calibrated models that include helium settling the present value of $Y_{e}$ is close to the values inferred from helioseismology. 
Despite the problems of separating effects of composition and equation of state. the results shown here demonstrate that current observations of solar uscillations are sensitive to aspects of the equation of state beyond the inclusion of the (oulomb effects. This offers hope that these data, and the substantially more accurate data expected from new helioseismic experiments, will provide new physical insight into the properties of the thermodynamics of hot partially ionized gases.

\subsection{Matching the near-surface effects}

Figure 28 indicates that with the inclusion of helium diffusion and settling the interior structure of solar models is very close to the that of the Sun. Also, the results in Section 5.2 suggested that the MHD equation of state provides a reasonable representation of the thermodynamics of the solar convection zone. To illustrate how these properties are reflected in the comparison between observed and computed frequencies. Figure $33(a)$ shows scaled frequency differences beiween the Sun and a model including helium settling, computed with the MHD cquation of state (Basu $/$ al. 1995). It is obvious that there is little evidence here for errors in the interior of the model, as would have been indicated by an $l$-dependence of the scaled differences or a substantial difference at low frequency. The only significant exception is probably at very high degree where the assumption of vertical propagation in the near-surface layers breaks down (c.g. Antia 1995; see also equation 33). The remaining scatter is likely to be predominantly observational.

I argued in Section 2.5 that the frequency-dependent difference could derive from a number of errors in the model or frequency computations. As a simple illustration of the effects of ncar-surface modifications on the comparison between observations and models I here consider a model using the same physics as for Figure 33(a), except that the opacity has been increased by a factor 2.34 at temperatures below about $8000 \mathrm{~K}$. The resulting scaled frequency differences are shuw11 in Figure $33(b)$. The change in atmospheric structure resulting from the opacity increase has clearly eliminated much of difference between observations and model; similar effects were also noted by Christensen-Dalsgaard (1990), Kim, Demarque \& Guenther (1991) and Turck-Chièze \& Lopes (1993). However, it is evident that there remain significant variations. Some of these are undoubtedly associated with the remaining differences between the interiors of the Sun and the model, illustrated in Figure 28. In addition, there is an indication of an oscillatory variation with frequency which might be related to errors in the equation of state or convection-zone helium abundance, as well as a contribution varying more slowly with frequency and hence probably concentrated very near the surface. 

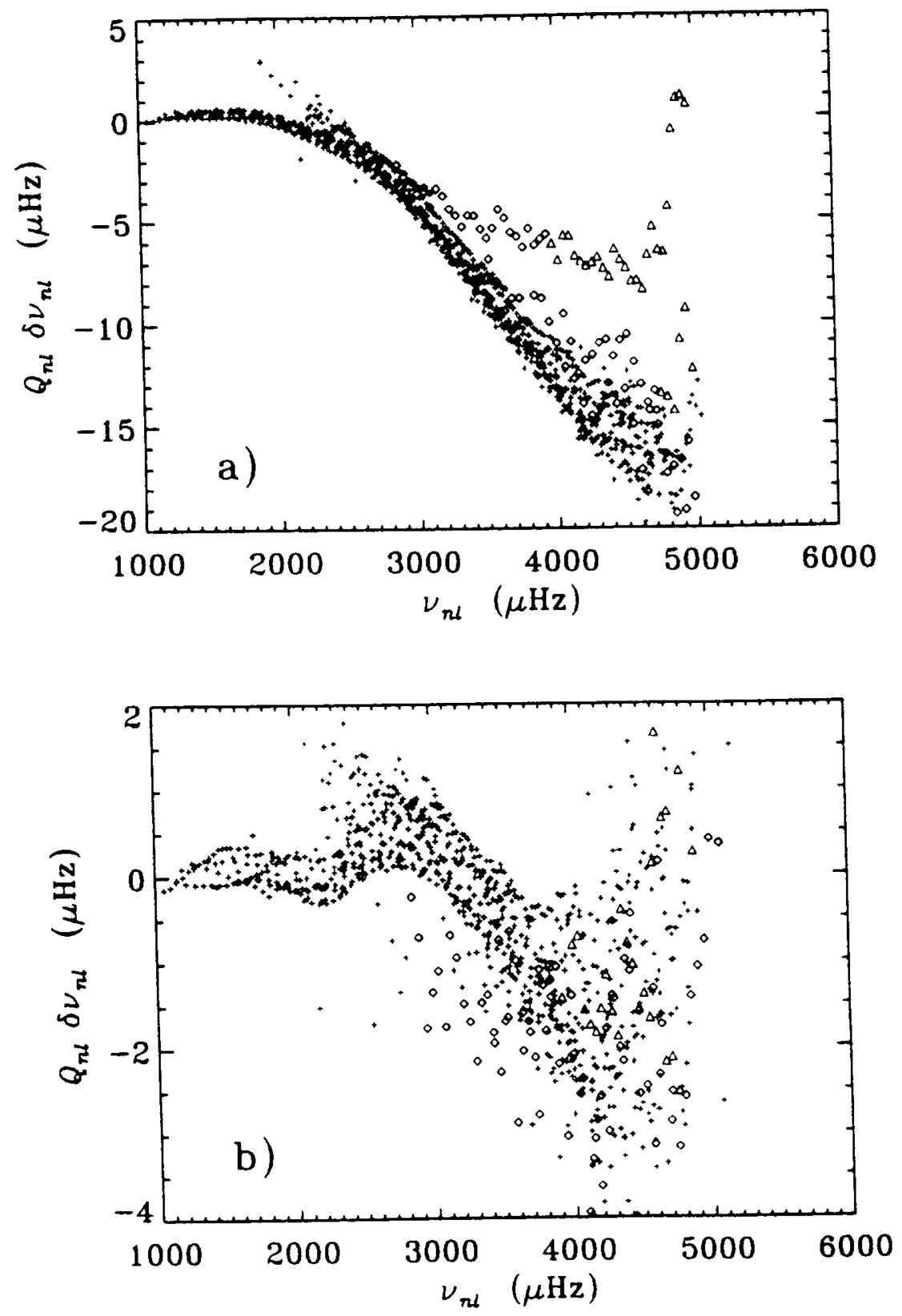

Figure 33: (a) Scaled differences between observed frequencies and frequencies of a model including helium diffusion and settling, computed with the MHD equation of state. Crosses indicated modes with $l \leq 500$, diamonds are modes with $500<l \leq 1000$ and triangles are modes with $1000<l$. (b) As (a), but for a model where in addition the atmospheric opacity has been increased by a factor 2.34 . 
It should be stressed that opacity errors of this magnitude in recent tables are quite unlikely. Thus this calculation cannot be regarded as a realistic attempt 1.) explain the frequency-dependent part of the difference between observations and models. Instead, it is presented here as a simple example of the effects of near-surface changes. Similar effects arise from other types of modifications. It was shown in Section 4.1 that the observed behaviour can be mimicked by making the superadiabatic gradient steeper, as in, for example, the Canuto \& Mazzitelli (1991) formulation (f.g. Paternó el al. 1993; Monteiro et al. 1995ab; cf. Figure 8). Superficially similar effects arise when turbulent pressure is taken into account in the equilibrium nodel, or from effects of convective fluctuations (e.g. Rosenthal et al. 1995b), while the effects of nonadiabaticity or the perturbation in the turbulent pressure are still somewhat uncertain, due to the difficulties in modelling the effects of convection (e.g. Christensen-Dalsgaard (.) Frandsen 1983; Cox. Cuzik \& Kidman 1989; Balmforth 1992b; Guenthrr 1994; Rosenthal et al. 1995b).

The situation concerning the near-surface problems in the modelling is clearly rather unsatisfactory at present. However, hope is provided by the availability of increasingly realistic hydrodynamical simulations of this region, accompanied by a better physical understanding of the relevant processes. Also, the expected more accurate observations may permit us to distinguish between the different models proposed to account for the frequency behaviour. Finally, potentially very important information concerning convective effects might be obtained from observations of solar-like oscillations in other stars covering a range in effective temperature and surface gravity.

\section{TOWARDS THE STARS?}

It is evident that observations of solar oscillations are providing extremely detailed information about the properties of the solar interior. This gives a precise test of stellar evolution theory, including the physical information that enter into it, as applied to the Sun. However, the Sun is only a single specific example of a star, with a comparatively simple structure. It is obviously of great interest to obtain similar information about other stars.

This encounters two problems. Detection of small-amplitude oscillations, such as those observed in the Sun, is greatly complicated by the low light-level available for stars other than the Sun. And the apparent extent of such stars is so small that essentially no spatial resolution is possible; thus with few exceptions the observations are limited to modes of low degree, as in whole-disk observations of solar oscillations (see Leibacher, this volume). As a result, the data for 
any individual remote star will always be much less extensive than the data available for the Sun. This, however, must be balanced against the possibility of studying stars of greatly varying parameters, such as mass and age, covering a corresponding range of physical properties and phenomena. A very important example are convective cores which are only found in stars more massive than the Sun: the properties of such convective cores and the associated mixing, which may include overshooting or weaker turbulence, are highly uncertain; yet such processes play a major rôle for the evolution of the stars on the main sequence and beyond.

The early analyses of this nature predates the first results of helioseismology. For a long time there appeared to be discrepancies between the observed periods of classical Cepheids and evolution models of these stars; the problem was particularly acute for double-mode Cepheids, as first pointed out by $\mathrm{Pe}$ tersen (1973). This and other discrepancies between pulsation observations and evolution calculations were reviewed by Cox (1980). The new computations of opacities, where a more careful treatment of lines has led to very substantial opacity increases (see Section 2.2.2), have largely solved these so-called "Cepheid mass problems" (e.g. Moskalik, Buchler \& Marom 1992; Kanbur \& Simon 1994; Christensen-Dalsgaard \& Petersen 1995). It is interesting that the effects in the Cepheids are dominated by the opacity at temperatures between $10^{5}$ and $10^{6} \mathrm{~K}$ : this temperature range falls within the solar convection zone and the corresponding opacities have no effect on solar structure. Thus studies of the double-mode Cepheids complement the information that can be obtained about opacity from helioseismology.

Extensive data are now available for pulsating white dwarfs (for a review, see e.g. Winget 1991), providing precise measures of white-dwarf masses, information about the thickness of the outer hydrogen layer, constraints on the rotation rate and magnetic field and in some cases measurements of evolutionary effects, visible as frequency changes. On or just after the main sequence observations of $\beta$ Cephei stars and other pulsating $B$ stars are providing information about the properties of relatively massive stars (e.g. Gautschy 1990); the recent opacity revisions have provided a natural explanation for the excitation of oscillations in these stars (e.g. Cox et al. 1992; Kiriakidis, El Eid \& Glatzel 1992; Moskalik \& Dziembowski 1992). Also, extensive sets of frequencies, which may even include $\mathrm{g}$ modes, are becoming available for $\delta$ Scuti stars (e.g. Breger et al. 1993; Belmonte et al. 1994; Frandsen et al. 1995; for a review, see Matthews 1993). Both $B$ stars and $\delta$ Scuti stars have convective cores and hence promise information that is unavailable for the Sun. Furthermore, by solar standards the amplitudes are large, with relative intensity variations of order $10^{-3}$, making the oscillations relatively easy to detect; even so, the identification of the modes still give rise to considerable uncertainty. Finally, the rapidly oscillating Ap stars (e.g. Kurtz 1995) display frequency spectra with some superficial similarity to the solar os- 
cillations of low degree, although strongly affected by the large-scale magnetic fields of these stars.

Even though a variety of stars are therefore good targets for asteroseismic investigations, it would clearly be of particular value to detect and study solarlike oscillations in stars other than the Sun. The diagnostic potential of such data is relatively well understood (Ulrich 1986; (hristensen-Dalsgaard 1984, 1988. 1993; Gough \& Novotny 1993; Brown et al. 1994; Audard, Provost \& ('hristensen-Dalsgaard 1995). Furthermore, information on the dependence of the nocke amplitudes and line widths on stellar parameters would provide important information about the excitation and damping processes, eventually perhaps leading to a hetter understanding of the properties of outer stellar convection zones. 'The detection of such oscillations has been elusive, however. So far a number of suggestive results have been obtained (e.g. Gelly, Grec \& Fossat 1986; Innis et al. 1991; Brown ct al. 1991; Pottasch, Butcher \& van Hoesel 1992), although with no definitive identification of oscillations. Also, a very ambitious project involving a substantial number of large telescopes reached a very low detection threshold but still failed to make definitive detection (Ciilliland of al. 1993). Basic problems in these attempts have been atmospheric noise in hroad-band intensity measurements and photon noise and spectrograph stability in velority measurements.

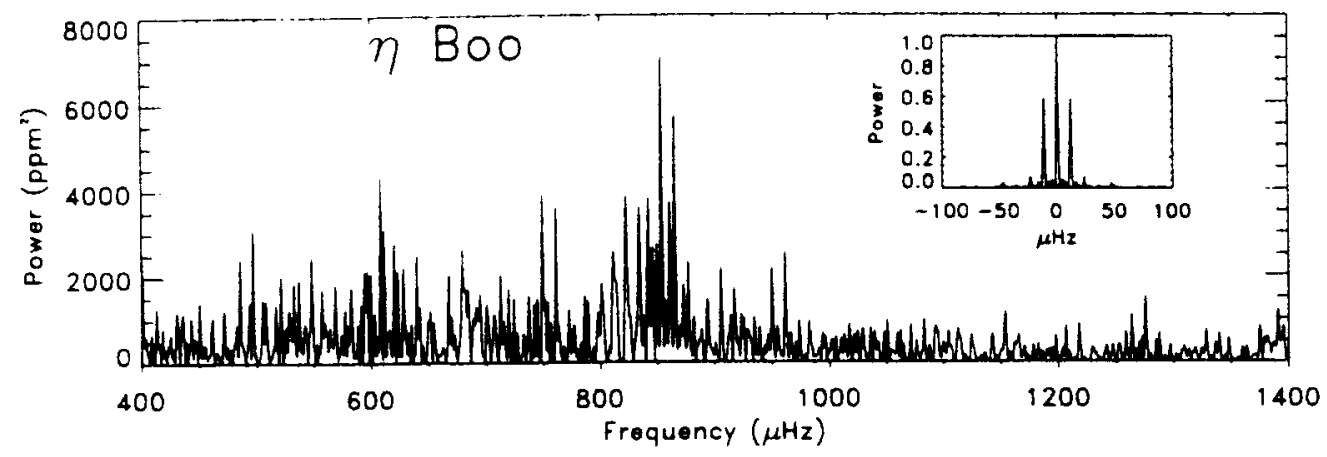

Figure 34: Observed power spectrum of $\eta$ Bootis. The inset shows the window function. (From Bedding \&i Kjeldsen 1995).

\section{$6.1 \eta$ Bootis}

The atmospheric effects can to a large extent be eliminated by making differential measurements, comparing the intensity in spectral lines with the intensity in the neighbouring continuum. Kjeldsen et al. (1995) showed that the integrated intensity in the Balmer lines, expressed in terms of the equivalent width, 
provides a sensitive measure of oscillations in stellar atmospheres. In this way they were able to detect probable solar-like oscillations in the sub-giant star $\eta$ Bootis, from 6 nights of observations with the $2.5 \mathrm{~m}$ Nordic Optical Telescope. Figure 34 shows the relevant part of the resulting power spectrum. There are clear indications of excess power in the frequency range $700-950 \mu \mathrm{Hz}$, of a shape superficially similar to the amplitude distribution in the solar five-minute oscillations.

The interpretation of the spectrum is greatly complicated by the presence of strong daily side lobes. Through a correlation analysis Kjeldsen et al. determined the large frequency separation $\Delta \nu$ (cf. equation 34) as $\Delta \nu \simeq 40.3 \mu \mathrm{Hz}$. To determine individual frequencies they carried out a so-called CLEAN analysis on the spectrum. Such techniques are subject to considerable uncertainty in data with comparatively low duty cycle; in particular, there is some risk of mistaking a side lobe for the main peak. However, Kjeldsen et al. inferred frequencies of thirteen modes, including several closely-spaced pairs which they identified as having $l=0$ and 2 , in accordance with the asymptotic expression (34).

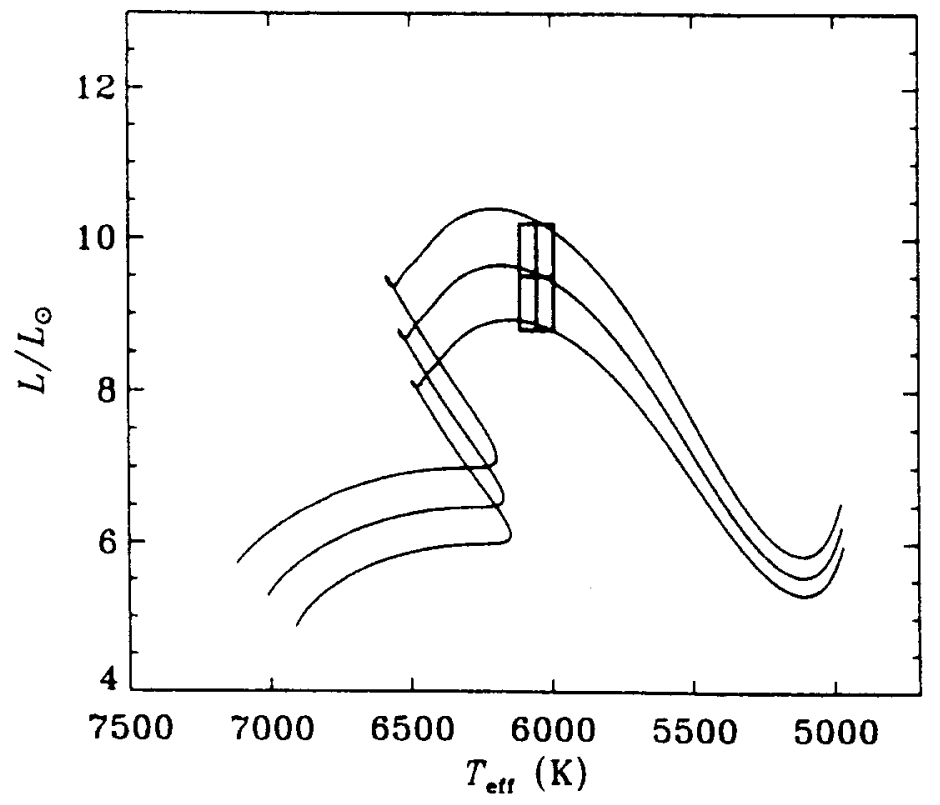

Figure 35: Evolutionary tracks in the $H R$ diagram, for models with $Z=0.03$, $X=0.7$ and a mixing-length parameter calibrated to obtain the proper solar radius. Models are shown with masses of $1.6 \mathrm{M}_{\odot}, 1.63 \mathrm{M}_{\odot}$ and $1.66 \mathrm{M}_{\odot}$. The error box indicates the observed location of $\eta$ Bootis. (Adapted from ChristensenDalsgaard et al. 1995b).

The interpretation of these data was considered by Christensen-Dalsgaard, 
Bedding \& heldsen (19951)). The star is sufficiently close that its distance is known with reasonable precision; from this its luminosity can be determined as $L=9.5 \pm 0.7 \mathrm{~L} .$. . Also, spectroscopy shows that the effective temperature is $T_{\text {eff }}=60.50 \pm 60 \mathrm{~K}$ and that the heavy-element abundance is somewhat higher than solar. Figure 35 shows the location of the star in a Hertzsprung-Russell diagram. together with evolutionary tracks for $Z=0.03$ and three masses. These identify the star as being past the phase of central hydrogen burning, and with a mass of about 1.6 $\mathrm{M}_{1-.}$. Calculation of adiabatic frequencies shows that it is possible to find models in the error box with a $\Delta \nu$ which is consistent with the observed value. This provides an excellent test of the consistency of the frequency observations with the more classical stellar data: $\Delta \nu$ is essentially proportional to the the characteristic dynamical frequency $\omega_{\mathrm{dyn}} \equiv\left(G M / R^{3}\right)^{1 / 2}$ and linnce is predominantly determined by the stellar radius; thus it is largely fixed by the location of the star in the HR diagram. On the other hand, this property also indicates that $\Delta \nu$ is relatively insensitive to the details of the stellar internal structure.

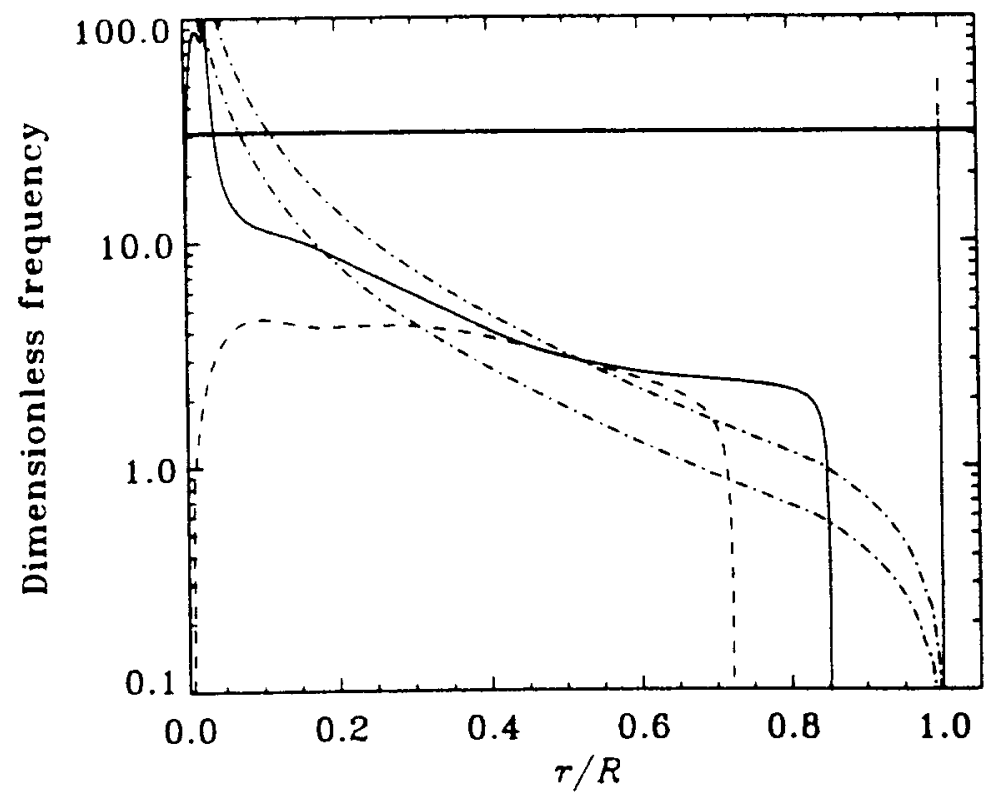

Figure 36: Dimensionless buoyancy frequency $\tilde{N} \equiv\left(G M / R^{3}\right)^{-1 / 2} N$ plotted against fractional radius $r / R$ for a model of the present $S u n$ (dashed line) and a model of $\eta$ Bootis (solid line). The dot-dashed lines show the dimensionless characteristic acoustic frequency $\bar{S}_{l}=\left(G M / R^{3}\right)^{-1 / 2} S_{l}$, where $S_{l}=c L / r$, for $l=1$ and 2 in $\eta$ Bootis. The heavy horizontal line indicates the location of a mode in $\eta$ Bootis of frequency $850 \mu \mathrm{Hz}$, typical of the observed frequencies.

To assist the understanding of the behaviour of the oscillations in $\eta$ Bootis, Figure 36 shows the buoyancy frequency and characteristic acoustic frequencies 
in a model of $\eta$ Bootis, in units of $\omega_{\mathrm{dyn}}$, and compare them with the buoyancy frequency in the present Sun. The dominant difference between the two models is the very large peak in $\tilde{N}$ near the centre of the $\eta$ Bootis model. This is caused by two effects: during main-sequence evolution the retreating convective core leaves behind a steep gradient in the hydrogen abundance, leading to a highly stable stratification and hence contributing to a large value of $N$ (e.g. Dziembowski \& Pamyatnykh 1991; Audard, Provost \& Christensen-Dalsgaard 1995); in addition, the increasing central condensation as the core contracts after hydrogen exhaustion drives up the gravitational acceleration in the core, further increasing $N$. As a result, the maximum value of $N$ exceeds the acoustical cutoff frequency in the stellar atmosphere. Thus all trapped acoustic modes may in principle be affected by the buoyancy frequency, taking on g-mode character in the core.

It should be recalled (see Leibacher, this volume) that a given mode behaves like a $\mathrm{p}$ mode where its frequency $\omega$ satisfies $\omega>N$ and $\omega>S_{l}$ and like a $\mathrm{g}$ mode where $\omega<N$ and $\omega<S_{l}$; in regions where $N<\omega<S_{l}$ the mode is evanescent, either decreasing or growing exponentially. Thus, at the frequencies characteristic for the observations of $\eta$ Bootis, indicated by the horizontal line in Figure 36, the modes have extended p-mode regions in the outer parts of the star and a small $\mathrm{g}$-mode region near the centre. The separation between these two regions is quite small for $l=1$, leading to a substantial coupling between the two types of behaviour; with increasing $l$, the separation increascs rapidly and the coupling becomes small.

The effects of this structure on the oscillations are illustrated in Figure 37. The frequencies of the radial modes, shown by dashed lines in panel $(a)$, decrease approximately with $\omega_{\mathrm{dyn}}$ as a result of the increasing stellar radius. The same general trend is shared by the $l=1$ modes when they behave like p modes. However, the figure shows the presence in addition of g-inode branches, with frequencies increasing with age as the maximum value of $N$ increases. This leads to resonances where frequencies of modes of the same degree undergo avoided crossings instead of crossing (e.g. Aizenman, Smeyers \& Weigert 1977); on the other hand, there is no interaction between modes of different degree. The effect on the mode inertia $E$ normalized at the photospheric amplitude, defined in equation (53), is shown in panel $(b)$; for clarity two modes with $l=1$ have been indicated in both panels by triangles and diamonds, respectively, at the points corresponding to the models in the evolution sequence. Where the $l=1$ modes behave as $\mathrm{p}$ modes, their inertia is very close to that of a radial mode of similar frequency. However, the g-mode behaviour corresponds to an increase in the amplitude in the interior and hence in $E$. At the avoided crossing there is an interchange of character between the two interacting modes. (It should be noted that the density of models in the sequence is insufficient to resolve fully the variations with age in $E$, leading to the somewhat irregular behaviour in 

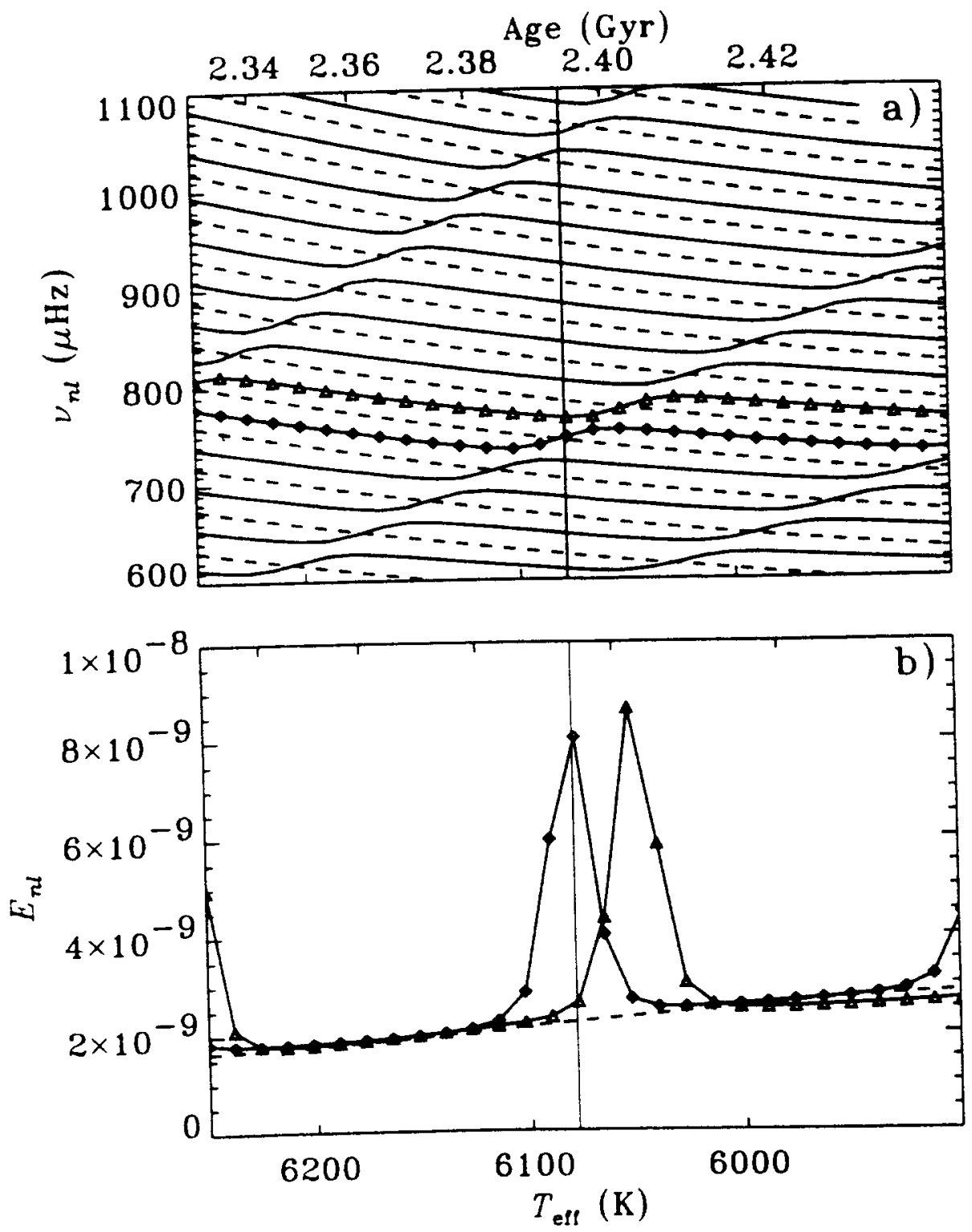

Figure 37: (a) Evolution of adiabatic frequencies for model of mass $1.60 \mathrm{M}_{\odot}$. The lower abscissa shows the effective temperature $T_{\text {eff }}$, the upper abscissa the age of the model in Gyr. The dashed lines correspond to modes of degree $l=0$, and the solid lines to $l=1$. The vertical solid line indicates the location of the model whose frequencies are illustrated in Figure 39. (Adapted from ChristensenDalsgaard et al. 1995b). (b) The change with age in the normalized mode inertia (cf. equation 53). The solid lines show modes with $l=1$, each model being indicated by triangles or diamonds as in panel (a), whereas the dashed line shows the radial mode with approximately the same frequency. 
panel (b); however, the overall variation is clearly visible.)

The properties of the oscillations are further illustrated by the eigenfunctions shown in Figure 38, for the two modes with $l=1$ undergoing avoided crossing at the vertical line in Figure 37 as well as for the neighbouring radial mode. The displacement amplitudes have been weighted by $\rho^{1 / 2} r$, so that they directly shows the contribution at a given radius to the mode inertia $E_{n l}$ ( $c f$. equation 53). The $l=1$ mode in panel $(a)$ is evidently very nearly a pure acoustic mode, with an vertical displacement behaving almost as for the radial mode, apart from the phase shift associated with the difference in frequency. In contrast, the second $l=1$ mode has very substantial displacement amplitudes in the core, leading to the comparatively large normalized inertia shown in Figure 37; this is particularly visible in the enlarged view in Figure 38(c). It should be noted, however, that the separation between the g-mode and p-mode propagation regions is quite small in this case (see also Figure 36), leading to substantial coupling between the two regions and causing the large minimum separation in the avoided crossing and a maximum normalized inertia which is still relatively small, despite the g-mode like behaviour in the core. In contrast, for modes with $l \geq 2$ the separation between the propagation regions is larger and the coupling is much weaker; as a result, a frequency plot corresponding to Figure 37(a) shows two sets of frequencies apparently crossing with no avoidance, and the maximum inertia for, for example, $l=2$ in the frequency region illustrated is around $3 \times 10^{-7}$.

The normalized inertia may provide a rough estimate of the likely surface amplitude of the modes, at least if the modes are excited stochastically by convection (e.g. Houdek et al. 1995): in that case the mode energy is likely to be independent of degree, at fixed frequency. It follows from equation (53) that kinetic energy of a mode can be expressed as $A^{2} E_{n l}$, where $A$ is the surface amplitude. Assuming that the energy is independent of degree, the amplitude $A_{n l}$ of a mode of degree $l$, order $n$ and normalized inertia $E_{n l}$ satisfies

$$
\frac{A_{n l}}{A_{0}\left(\nu_{n l}\right)} \simeq\left[\frac{E_{n l}}{\bar{E}_{0}\left(\nu_{n l}\right)}\right]^{-1 / 2},
$$

where $A_{0}(\nu)$ and $\bar{E}_{0}(\nu)$ are obtained by interpolating to frequency $\nu$ in the results for radial modes. In particular, the modes with strong g-mode character in Figure 37 would be expected to have roughly half the surface amplitude of the pure acoustic modes.

To compare the fine structure in the observed and computed frequency spectra it is convenient to use an echelle diagram (e.g. Grec, Fossat \& Pomerantz 1983). Here, the frequencies are reduced modulo $\Delta \nu$ by expressing them as

$$
\nu_{n l}=\nu_{0}+k \Delta \nu+\dot{\nu}_{n l}
$$



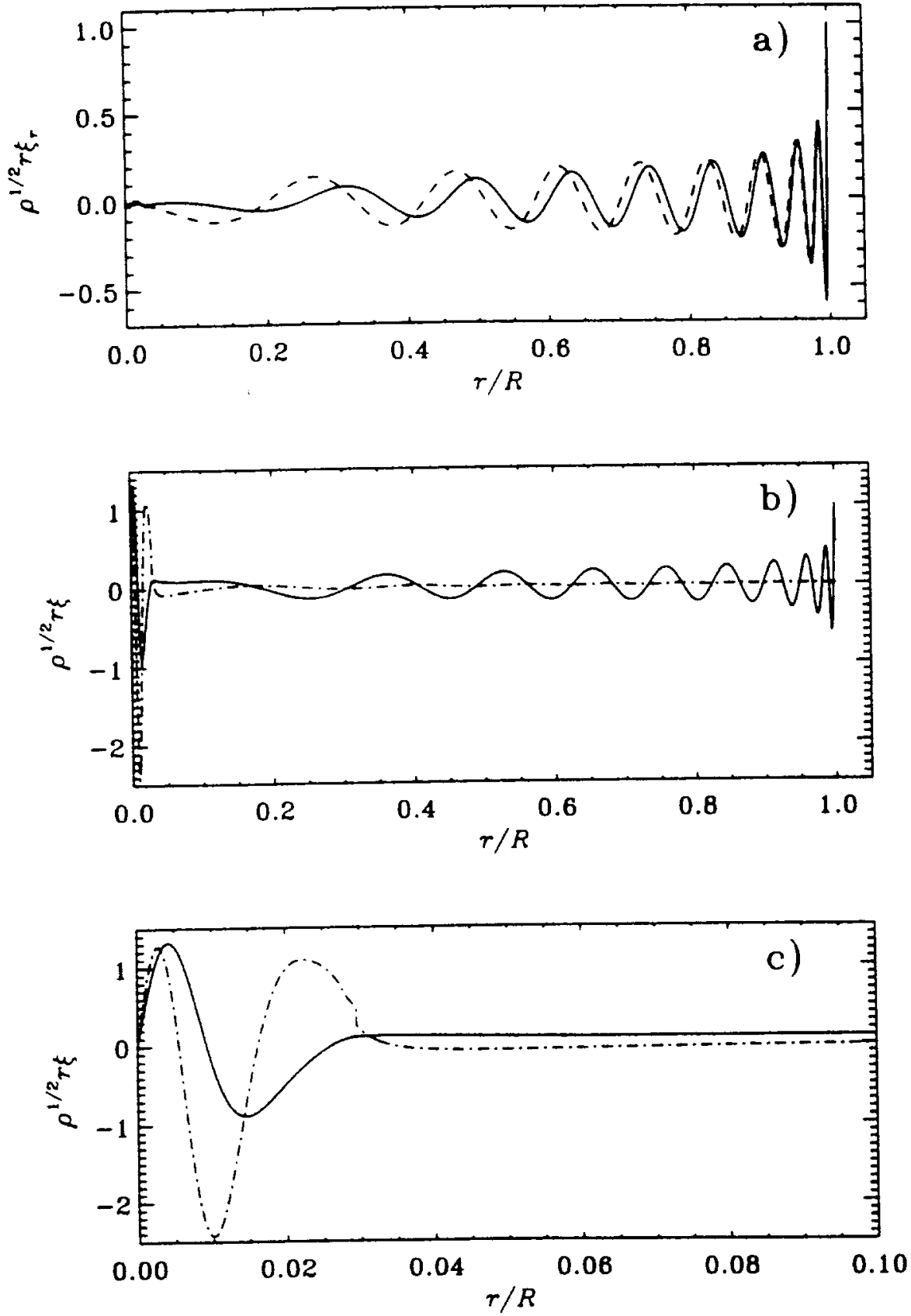

Figure 38: Eigenfunctions of selected modes in the model indicated by a vertical line in Figure 37. In panel (a) the amplitudes of the vertical displacement are shown for the $l=1$ mode indicated by triangles (solid line) and the neighbouring radial mode (dashed line). Panels (b) and (c) are for the $l=1$ mode marked by diamonds: the solid and dot-dashed lines show the amplitudes of the vertical and horizontal displacement, respectively. 


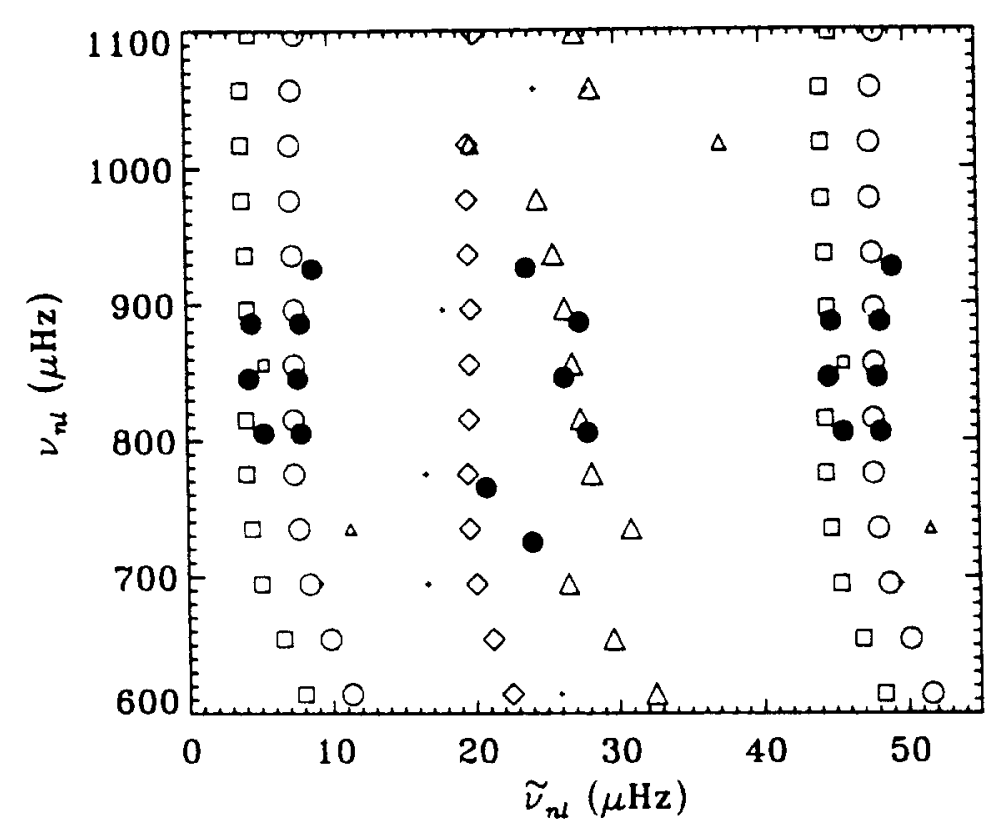

Figure 39: Echelle diagram with a frequency separation of $\Delta \nu=40.3 \mu \mathrm{Hz}$. The open symbols show computed frequencies for a model with $M=1.60 \mathrm{M}_{\odot}$ and $Z=0.03$; here the reference frequency was $\nu_{0}^{(\bmod )}=856 \mu \mathrm{Hz}$. Circles are used for modes with $l=0$, triangles for $l=1$, squares for $l=2$ and diamonds for $l=3$. The size of the symbols indicates the expected relative amplitude of the modes (see text); symbols that would otherwise be too small have been replaced by crosses. The filled circles show observed frequencies from Kjeldsen et al. (1995), plotted with the same $\Delta \nu$ but with a reference frequency of $\nu_{0}^{(\text {obs })}=846 \mu \mathrm{Hz}$.

(where $\nu_{0}$ is a suitable reference frequency and $k$ is an integer), such that $\tilde{\nu}_{n l}$ is between 0 and $\Delta \nu$. In the echelle diagram $\nu_{0}+k \Delta \nu$ is plotted against $\tilde{\nu}_{n l}$. The result is shown in Figure 39 . The open symbols are for a $1.6 \mathrm{M}_{\odot}$ model that was chosen to have $\Delta \nu \simeq 40.3 \mu \mathrm{Hz}$; the reference frequency was $\nu_{0}^{\text {(mod) }}=$ $856 \mu \mathrm{Hz}$. The sizes of the symbols have been scaled by the amplitude ratio $A_{n l} / A_{0}$ determined by equation (68).

The model results for $l=0,2$ and 3 clearly reflect the behavior predicted by equation (34). In particular, the points for $l=0$ and 2 run parallel, with a small separation $\delta_{n 0} \simeq 3.3 \mu \mathrm{Hz}$ resulting from the last term in that equation. For $l=1$, equation (34) predicts an almost vertical series of points separated by roughly $\Delta \nu / 2$ from those for $l=0$. The model frequencies deviate from this. Comparison with Figure $37(a)$ (where the location of this model is marked by a vertical solid line) indicates that this behavior is associated with the avoided crossings, which change the frequency separation and therefore shift the frequencies relative 
to the location expected from p-mode asymptotics. As discussed above, even $l=1$ modes behaving partly like g modes still have sufficiently small normalized inertia $E_{n l}$ that their estimaticd amplitudes are close to those of the pure p modes. (The figure shows a single exception: a mode at $730 \mu \mathrm{Hz}$ shifted almost to the $l=0$ line, with somewhat reduced amplitude.) In contrast, since the g modes of legree 2 and 3 are trapped quite efficiently in the deep interior, their estimated amplitudes are so small as to make the points virtually invisible in Figure 39.

The filled circles in Figure 39 show the frequencies observed by Kjoldsen et al. (1995), again plotted with $\Delta \nu=40.3 \mu \mathrm{Hz}$, but with the reference frequency $\nu_{0}^{(n, s)}=8.46 \mu H_{z}$. We can immediately identify modes with degrees $l=0$ and 2 . and the small frequency separation found by kjeldsen et al. $\left(\delta_{n 0}=3.1 \pm\right.$ $0.3 \mu \mathrm{H} z)$ is in excellent agreement with the model value. The remaining six observed frequencies coincide quite well with $l=1$ modes in the model and display an irregularity similar to the model frequencies (although differing in (retail). This might indicate that the observations of $\eta$ Bootis show evidence for avoided crossings involving g modes. Note, however, that some of the observed frecpuencies may arise from modes with $l=3$.

The interpretation of the observations must clearly be regarded as preliminary, given the uncertainty in extracting individual frequencies in a single-site power spectrum complicated by side lobes. In particular, the indications in the echelle diagram of effects of g-mode trapping is clearly extremely tentative. On the other hand, the close agreement between the observed and computed value of the $l=0-2$ frequency separation is suggestive. The difference of $10 \mu \mathrm{Hz}$ in the reference frequency $\nu_{0}$ required to obtain agreement between the location of the modes in Figure 39 is clearly a concern; however, it should be noted that this is of a similar magnitude to the differences observed in comparisons of solar observed and computed frequencies and attributed to errors in the treatment of the superficial layers. Sinilar effects might be expected for $\eta$ Bootis (see also ('hristensen-Dalsgaard ct al. 1995c).

\subsection{Concluding remarks}

The helioseismic investigations of the solar interior have undergone a dramatic development since the initial determination of individual $p$-mode frequencies of the Sun more than 15 years ago. Inverse analyses now let us determine density and sound speed in most of the solar interior with a relative precision exceeding $10^{-3}$. Remarkably, the results are quite close to normal solar models, provided that gravitational settling and diffusion of helium are taken into account, although significant differences remain. The frequencies have been shown to be sensitive to fine details in the physics of the solar interior, particularly the ther- 
modynamic properties and the opacity; this was used early to indicate the need for opacity increases, later confirmed, and is permitting tests of sophisticated formulations of the equation of state against the Sun.

The study of solar oscillations is on the threshold of a new era, with the deployment of the GONG project (e.g. Harvey et al. 1993; Leibacher et al. 1995) and the instruments on the SOHO satellite, expected to be launched towards the end of in 1995 (e.g. Scherrer et al. 1995; A ppourchaux of al. 1995; Gabriel et al. 1991). Together with the existing BISON and IRIS networks (e.g. Elsworth et al. 1995; Fossat 1995) these projects will provide a major expansion of the helioseismic data. This should result in greatly improved information about the structure of the solar core, of obvious importance to the understanding of solar evolution and the neutrino problem, substantially better knowledge about conditions at the base of the convection zone, constraining possible overshooting and turbulent mixing in this region, and a much stronger basis for testing the equation of state and determining the envelope helium abundance. Furthermore, high-quality data on high-degree modes may improve our understanding of the properties of the near-surface region, including the effects of convection on the structure of the Sun and on the frequencies and excitation of the oscillations.

With the possible detection of modes in $\eta$ Bootis, asteroseismology of solarlike stars may now be in a position similar to helioseismology 15 years ago, with a corresponding promise for the future development. The technique developed by Kjeldsen et al. (1995) is being applied to other bright stars; an important example where extensive observations have already been obtained is $\alpha$ Centauri. Also, it is obvious that further observations of $\eta$ Bootis are required, to confirm and extend the initial results. Particularly important are multi-site observations, to reduce the complications of the side lobes in the spectrum. Also, the theoretical analysis in Section 6.1 indicates the substantial richness in the oscillations of sub-giant stars of somewhat more than solar mass, compared with stars in the phase of central hydrogen burning. Combined with their intrinsic brightness this makes subgiants attractive targets for asteroseismic investigations.

The effects of the Earth's atmosphere are likely to constrain ground-based investigations of solar-like oscillations to bright stars, observed with large telescopes. From space, however, the observations are limited essentially only by photon noise; here broad-band intensity measurements will allow the study of oscillations at solar amplitudes in fairly faint stars with modest-sized telescopes. An initial experiment of this nature, the EVRIS project (Baglin 1991), is scheduled for launch in November 1996 on the Russian Mars probe MARS96. It will use a $9 \mathrm{~cm}$ telescope to observe a limited number of rather bright stars during the 300-day cruise phase of the probe. Two more ambitious projects are currently under evaluation. The French COROT project (Catala et al. 1995) is aimed at providing extensive observations of a modest number of stars, to 
obtain highly accurate measurements of frequencies and frequency splittings. The STARS project, which is currently undergoing Phase A studies within ESA with a view towards possible selection in 1996 , will use an $80 \mathrm{~cm}$ telescope with a C CD detector to make simultaneous observations of several stars (Fridlund ft al. 1995). An important goal is to study solar-like oscillations of stars in open clusters: since stars in a cluster can be assumed to have approximately the same age, distance and chemical composition, measured frequencies for stars in a cluster provide far more stringent constraints on the internal structure of the stars than do frequencies of a single star, where the basic parameters are often rather uncertain.

Given the new helioseismic experiments, the continuing observations of "classical" pulsating stars and the growing potential for study of solar-like oscillations in other stars there seems little doubt that helio- and asteroseismology will remain a very active and fertile field of research for years to come.

\section{Acknowledgements}

I am very grateful to the organizers of the VI IAC Winter School for inviting me to participate in that splendid event. I thank the many colleagues who have contributed to the development of this field and my understanding of it, for enlightening discussions. The work presented here was supported in part by the Danish National Research Foundation through its establishment of the Theoretical Astrophysics Center.

\section{REFERENCES}

Ahrens, B., Stix, M. \& Thorn, M.: 1992, "On the depletion of lithium in the Sun", Astron. Astrophys. 264, 673 - 678.

Aizenman, M., Smeyers, P. \& Weigert, A.: 1977, "Avoided crossing of modes of non-radial stellar oscillations", Astron. Astrophys. 58, $41-46$.

Anders, E. \& Grevesse, N.: 1989, "Abundances of the elements: meteoritic and solar", Geochim. Cosmochim. Acta 53, 197 - 214.

Ando, H. \& Osaki, Y.: 1975, "Nonadiabatic nonradial oscillations: An application to the five-minute oscillation of the sun", Publ. Astron. Soc. Japan 27, $581-603$.

Ando, H. \& Osaki, Y.: 1977, "The influence of the chromosphere and corona on the solar atmospheric oscillations", Publ. Astron. Soc. Japan 29, 221 233 . 
Antia, H. M.: 1995, "Effects of surface layers on helioseismic inversion", Mon. Not. R. astr. Soc. 274, $499-503$.

Antia, H. M. \& Basu, S.: 1994, "Measuring the helium abundance in the solar envelope: the role of the equation of state", Astrophys. J. 426, 801-811.

Appourchaux, T., Domingo, V., Fröhlich, C., Romero, J., Wehrli, Ch., Andersen, B. N., Berthomieu, G., Delache, Ph., Crommelynck, D., Jiménez, A., Roca Cortés, T. \& Jones, A. R.: 1995, "VIRGO - The solar monitor experiment on SOHO", in R. K. Ulrich, E. J. Rhodes Jr \& W. Däppen (eds), Proc. GONG'94: Helio- and Astero-seismology from Earth and Space, ASP Conf. Ser. 76, $408-415$.

Audard, N., Provost, J. \& Christensen-Dalsgaard, J.: 1995, "Seismological effects of convective-core overshooting in stars of intermediate mass", Astron. Astrophys. 297, $427-440$.

Baglin, A.: 1991, "Stellar seismology from space: the EVRIS experiment on board MARS 94", Solar Phys. 133, 155 - 160.

Bahcall, J. N. \& Pinsonneault, M. H.: 1995, "Solar models with helium and heavy element diffusion", Rev. Mod. Phys., in the press.

Balmforth, N. J.: 1992a. "Solar pulsational stability. I: Pulsation-mode thermodynamics", Mon. Not. R. astr. Soc. 255, 603-631.

Balmforth, N. J.: 1992b. "Solar pulsational stability. II: Pulsation frequencies", Mon. Not. R. astr. Soc. 255, $632-638$.

Basu, S. \& Antia, H. M.: 1995, "Helium abundance in the solar envelope", Mon. Not. R. astr. Soc., in the press.

Basu, S., Antia, H. M. \& Narasimha, D.: 1994, "Helioseismic measurement of the extent of overshoot below the solar convection zone", Mon. Not. $R$. astr. Soc. 267, $209-224$.

Basu, S., Christensen-Dalsgaard, J., Schou, J., Thompson, J. \& Tomczyk, S.: 1995, "Solar structure inversion with LOWL data", in V. Domingo et al. (eds), Proc. 4th SOHO Workshop: Helioseismology, ESA SP-376, ESTEC, Noordwijk, in the press.

Bedding, T.R. \& Kjeldsen, H.: 1995, "More on solar-like oscillations in $\eta$ Boo", in R. S. Stobie \& P. A. Whitelock (eds), IAU Colloquium 155: Astrophysical Applications of Stellar Pulsation, ASP Conf. Ser., in the press.

Belmonte, J. A., Michel. E., Alvarez, M., Jiang, S. Y., Chevreton, M., Auvergne, M., Liu, Y. Y., Goupil, M. J., Baglin, A., Roca Cortés, T., Mangeney, A., Dolez, N., Valtier, J. C., Massacrier, G., Sareyan, J. P., Schmider, F. X. \& Vidal, I.: 1994, "Time-resolved photometry of BN and BU Cancri, two $\delta$ Scuti stars in the Praesepe cluster. Fourth photometry campaign of the STEPHI network", Astron. Astrophys. 283, 121 - 128.

Berthomieu, G., Cooper, A. J., Gough, D. O., Osaki, Y., Provost, J. \& Rocca, A.: 1980, "Sensitivity of five minute eigenfrequencies to the structure of the Sun", in H. A. Hill \& W. Dziembowski (eds), Nonradial and nonlinear stellar pulsation, Lecture Notes in Physics 125, Springer-Verlag, Berlin, 307 $-312$ 
Breger. M., Stich. I.. Garrido, R., Martiu. B., Shi-yang, Jiang, Zhi-ping, Li, Hube, D. P., Ostermann, W., Paparo. M., Schreck, M.: 1993, "Nonradial pulsation of the $\delta$ Scuti star BU Cancri in the Praesepe cluster", Astron. Astrophys. 271, 482486.

Brodsky, M. A. \& Vorontsov, S. V.: 1993, "Asymptotic theory of intermediateand high-degree solar acoustic oscillations", Astrophys. J. 409, 455-464.

Brookes, J. R., Isaak, G. R. \& van der Raay, H. B.: 1976, "Observation of free oscillations of the Sun", Nature 259, $92-95$.

Brown, T. M. ('hristensen-Dalsgaard, J.. Mihalas. B. \& Gilliland, R. L.: 1994, "The effectiveness of oscillation frequencies in constraining stellar model parameters". Astrophys. J. 427, 1013 - 1034.

Brown. T. M., Gilliland, R. L., Noyes, R. W. \& Ramsey, L. W.: 1991, "Detection of possible p-mode oscillations of Procyon". Astrophys. J. 368, $599-609$.

(anuto, V. M. \& Mazzitelli, I.: 1991. "Stellar turbulent convection: a new model and applications", Astrophys. J. 370, 295311

Canuto, V. M. \& Mazzitelli, I.: 1992, "Further improvements of a new model for turbulent convection in stars", Astrophys. J. 389, 724-730.

Catala, C.. Auvergne, M., Baglin, A., Bonneau, F., Magnan, A., Vuillemin, A., Coupil, M. J., Michel. E., Boumier, P., Dzitko, H., Gabriel, A., Gautier, D.. Lemaire, P., Mangeney, A., Mosser, B.. Turck-Chièze, S. \& Zahn, J. P.: 1995, "COROT, a space project devoted to the study of convection and rotation in stars", in V. Domingo et al. (eds), Proc. 4th SOHO Workshop: Helioseismology, ESA SP-376, ESTEC. Noordwijk, in the press.

Chaboyer, B., Demarque. P., Guenther, D. B. \& Pinsonneault, M. H.: 1995, "Rotation, diffusion and overshoot in the Sun: effects on the oscillation frequencies and the neutrino flux", Astrophys. J. 446, $435-444$.

Christensen-Dalsgaard, J.: 1981, "The effect of non-adiabaticity on avoided crossings of non-radial stellar oscillations". Mon. Not. R. astr. Soc. 194, $229-250$.

Christensen-Dalsgaard, J.: 1981, "What will asteroseismology teach us?", in F. Praderie (ed.), Space Research Prospects in Stellar Activity and Variability, Paris Observatory Press. 11 - 45.

Christensen-Dalsgaard, J.: 1986, "Theoretical aspects of helio- and asteroseismology", in D. O. Gough (ed.), Seismology of the Sun and the distant Stars, iteidel, Dordrecht, 23 - 53.

Christensen-Dalsgaard, J.: 1988, "A Hertzsprung-Russell diagram for stellar oscillations", in J. Christensen-Dalsgaard \& S. Frandsen (eds), Proc. IAU Symposium No 123, Advances in helio- and asteroseismology, Reidel, Dordrecht, 295 - 298.

Christensen-Dalsgaard, J.: 1990, "Helioseismic investigation of solar internal structure", in G. Berthomieu \& M. Cribier (eds), Proc. IAU Colloquium No 121, Inside the Sun, Kluwer, Dordrecht, $305-326$.

Christensen-Dalsgaard, J.: 1991, "Some aspects of the theory of solar oscillations", Geophys. Astrophys. Fluid Dynamics 62, $123-152$. 
Christensen-Dalsgaard, J.: 1992, "Solar models with enhanced energy transport in the core", Astrophys. J. 385, $354-362$.

Christensen-Dalsgaard, J.: 1993, "On the asteroseismic HR diagram", in T. M. Brown (ed.), Proc. GONG 1992: Seismic investigation of the Sun and stars, ASP Conf. Ser. 42, $347-350$

Christensen-Dalsgaard, J.: 1994, Lecture Notes on Stellar Oscillations (Third Edition), D.f.I. Print, Aarhus.

Christensen-Dalsgaard, J. \& Däppen, W.: 1992, "Solar oscillations and the equation of state", Astron. Astrophys. Rev. 4, $267-361$.

Christensen-Dalsgaard, J. \& Frandsen, S.: 1983, "Radiative transfer and solar oscillations", Solar Phys. 82, 165 - 204.

Christensen-Dalsgaard, J. \& Gough, D. O.: 1976, "Towards a heliological inverse problem", Nature 259, 89 - 92 .

Christensen-Dalsgaard, J. \& Gough, D. O.: 1980, "Is the Sun helium-deficient?", Nature 288, 544 - 547.

Christensen-Dalsgaard, J. \& Gough, D. O.: 1984, "Implications of observed frequencies of solar p modes", in R. K. Ulrich, J. Harvey, E. J. Rhodes Jr \& J. Toomre (eds), Solar Seismology from Space, NASA, JPL Publ. 84-84, $199-204$,

Christensen-Dalsgaard, J. \& Pérez Hernández, F.: 1991, "Influence of the upper layers of the Sun on the p-mode frequencies", in D. O. Gough \& J. Toomre (eds), Challenges to theories of the structure of moderate-mass stars, Lecture Notes in Physics 388, Springer, Heidelberg, $43-50$.

Christensen-Dalsgaard, J. \& Pérez Hernández, F.: 1992, "Phase-function differences for stellar acoustic oscillations - I. Theory", Mon. Not. R. astr. Soc. 257, 62 - 88 .

Christensen-Dalsgaard, J. \& Petersen, J. O.: 1995, "Pulsation models of the double-mode Cepheids in the Large Magellanic Cloud", Astron. Astrophys., in the press.

Christensen-Dalsgaard, J., Duvall, T. L., Gough, D. O., Harvey, J. W. \& Rhodes Jr, E. J.: 1985, "Speed of sound in the solar interior", Nature 315, 378 382.

Christensen-Dalsgaard, J., Däppen, W. \& Lebreton, Y.: 1988, "Solar oscillation frequencies and the equation of state", Nature 336, 634-638.

Christensen-Dalsgaard, J., Gough, D. O. \& Pérez Hernández, F.: 1988, "Stellar disharmony", Mon. Not. R. astr. Soc. 235, $875-880$.

Christensen-Dalsgaard, J., Gough, D. O. \& Thompson, M. J.: 1989, "Differential asymptotic sound-speed inversions", Mon. Not. R. astr. Soc. 238, 481 502.

Christensen-Dalsgaard, J., Gough, D. O. \& Thompson, M. J.: 1991, "The depth of the solar convection zone", Astrophys. J. 378, $413-437$.

Christensen-Dalsgaard, J., Gough, D. O. \& Thompson, M. J.: 1992, "On the rate of destruction of lithium in late-type main-sequence stars", Astron. Astrophys. 264, 518 - 528 . 
('hristensen-Dalsgaard, J.. Proffitt, C. R. \& 'Thompson, M. J.: 1993, "Effects of diffusion on solar models and their oscillation frequencies", Astrophys. J. 403. L75 - L78.

('hristensen-Dalsgaard, J.. Monteiro, M. J. P. F. G. \& Thompson, M. J.: 1995a. "Helioseismic estimation of convective overshoot in the Sun", Mon. Not. $R$. astr. Soc, in the press.

('hristensen-Dalsgaard, J., Bedding, T. R. \& Kjeldsen, H.: 1995b. "Modelling solar-like oscillations in $\eta$ Bootis", Astrophys. J. 443, L29 - L32.

(hristensen-Dalsgaard. J.. Bedding. T'. R., Houdek, G., Kjeldsen, H., Rosenthal, ('.. Trampedach, R.. Monteiro, M. J. P. F. G. \& Nordlund, §.: 1995c. "Near-surface effects in modelling oscillations of $\eta$ Boo", in R. S. Stobie \& P. A. Whitelock (eds). IAU Colloquium 155: Astrophysical Applications of Stellar Pulsation. ASP Conf. Ser., in the press.

Claverie, A., Isaak, G. R., McLeod, C. P., van der Raay, H. B. \& Roca Cortes, 'T.: 1979, "Solar structure from global studies of the 5-minute oscillations", Nature 282, 591 - 644.

('ox, A. N.: 1980, "The masses of Cepheids", Ann. Rev. Astron. Astrophys. $18,15 \cdot 41$.

(ox, A. N., Guzik, J. A. ( Kidman, R. B.: 1989, "Oscillations of solar models with internal element diffusion", Astrophys. J. 342, $1187-1206$.

Cox, A. N., Morgan, S. M.. Rogers, F. J. \& Iglesias, C. A.: 1992, "An opacity mechanism for the pulsations of OB stars", Astrophys. J. 393, 272 - 277.

Däppen. W.: 1992, "The equation of state for stellar envelopes: comparison of results from different formalisms", in C. Lynas-Gray, C. Mendoza \& C. Zeippen (eds), Proc. Workshop on Astrophysical Opacities, Revista Mexicana de Astronomia y Astrofisica 23, 141 - 149.

Däppen, W. \& Gough, D. O.: 1984, "On the determination of the helium abundance of the solar convection zone", in Theoretical Problems in Stellar Stability and Oscillations, Institut d'Astrophysique, Liège, $264-269$.

Däppen. W. \& Gough, D. O.: 1986, "Progress report on helium abundance determination", in D. O. Gough (ed.), Seismology of the Sun and the distant Stars, Reidel, Dordrecht, $275-280$.

Demarque, P. \& Guenther, D. B.: 1988, "Sensitivity of solar p-modes to solar envelope structure", in J. Christensen-Dalsgaard \& S. Frandsen (eds), Proc. IAU Symposium No 123, Advances in helio- and asteroseismology, Reidel, Dordrecht, 91 - 94 .

Deubner, F.-L.: 1975, "Observations of low wavenumber nonradial eigenmodes of the Sun", Astron. Astrophys. 44, $371-375$.

Deubner, F.-L. \& Gough, D. O.: 1984, "Helioseismology: Oscillations as a diagnostic of the solar interior", Ann. Rev. Astron. Astrophys. 22, 593 619.

Duvall, T. L., Dziembowski, W. A., Goode, P. R., Gough, D. O., Harvey, J. W. \& Leibacher, J. W.: 1984, "The internal rotation of the Sun", Nature 310, $22-25$. 
Dyson, J. \& Schutz, B. F.: 1979, "Perturbations and stability of rotating stars. I. Completeness of normal modes", Proc. Roy. Soc. London A368, 389 410.

Dziembowski, W. A. \& Pamjatnykh, A. A.: 1991, "A potential asteroseismological test for convective overshooting theories", Astron. Astrophys. 248, L11 - L14.

Dziembowski, W. A., Pamyatnykh, A. A. \& Sienkiewicz, R.: 1992, "Seismological tests of standard solar models calculated with new opacities", Acta Astron. 42, 5 - 15.

Dziembowski, W. A., Goode, P. R., Pamyatnykh, A. A. \& Sienkiewicz, R.: 1994, "Seismic model of the sun's interior", Astrophys. J. 432,417-426.

Eddington, A. S.: 1926, The internal constitution of the stars, Cambridge University Press, Cambridge.

Eggleton, P. P., Faulkner, J. \& Flannery, B. P.: 1973, "An approximate equation of state for stellar material", Astron. Astrophys. 23, 325-330.

Eisenfeld, J.: 1969, "A completeness theorem for an integro-differential operator", J. Math. Anal. Applic. 26, $357-375$.

Elsworth, Y., Howe, R., Isaak, G. R., McLeod, C. P. \& New, R.: 1990, "Evidence from solar seismology against non-standard solar-core models", Nature $\mathbf{3 4 7}$, $536-539$.

Elsworth, Y., Howe, R., Isaak, G. R., McLeod, C. P., Miller, B. A., New, R., Speake, C. C. \& Wheeler, S. J.: 1994, "Solar p-mode frequencies and their dependence on solar activity: recent results from the BISON network", Astrophys. J. 434, $801-806$.

Elsworth, Y., Howe, R., Isaak, G. R., McLeod, C. P., Miller, B. A., van der Raay, H. B. \& Wheeler, S. J.: 1995, "Performance of the BISON network 1981-present", in R. K. Ulrich, E. J. Rhodes Jr \& W. Däppen (eds), Proc. GONG'94: Helio- and Astero-seismology from Earth and Space, ASP Conf. Ser. 76, $392-397$.

Faulkner, J. \& Gilliland, R. L.: 1985, "Weakly interacting, massive particles and the solar neutrino flux", Astrophys. J. 299, $994-1000$.

Fossat, E.: 1995, "IRIS status report", in R. K. Ulrich, E. J. Rhodes Jr \& W. Däppen (eds), Proc. GONG'94: Helio- and Astero-seismology from Earth and Space, ASP Conf. Ser. 76, 387 - 391.

Fossat, E. \& Ricort, G.: 1975, "Photospheric oscillations. I. Large scale observations by optical resonance method", Astron. Astrophys. 43, 243 252.

Frandsen, S., Balona, L. A., Viskum, M., Koen, C. \& Kjeldsen, H.: 1995, "Multisite CCD photometry of $\delta$ Scuti stars in the open cluster NGC6134 (the 1. STACC campaign)", submitted to Astron. Astrophys..

Fridlund, M., Gough, D.O., Jones, A., Appourchaux, T., Badiali, M., Catala, C., Frandsen, S., Grec, G.. Roca Cortés, T. \& Schrijver, K.: 1995, "STARS: A proposal for a dedicated space mission to study stellar, structure and evolution", in R. K. Ulrich, E. J. Rhodes Jr \& W. Däppen (eds), Proc. 
Testing a solar model: the forward problem

CONC99: Helio- and Astero-seismology from Earth and Space, ASP Conf. Ser. $76,416425$.

(iabricl. A. H. and the (GOLF team: 1991. "(ilobal oscillations at low frequency from the SOHO mission (GOLF)", Adv. Space Res. vol. 11, No. 4, 103 112.

(iabriel. M.: 1989, "The $D_{n}$ values and the structure of the solar core", Astron. Astrophys. 226, 278 28:3.

(iabriel, M., Scuflaire. R. (L Noels: 1982, "The solar structure and the low $l$ five-minute oscillation. I", Astron. Astrophys. 110,50-53.

(iautschy, A.: 1990, "On the pulsation evolution connection of early-type stars", in D. Baade (col.). Proc. ESO Workshop on Rapid variability of OB-stars: nature and disgnostic nalue, ESO Munich, 315 - 329.

(ielly. B., Grec, G. \& Fossat. E.: 1986, "Evidence for global pressure oscillations in Procyon and $\alpha$ Centauri", Astron. Astrophys. 164, $383-394$.

(iilliland. R. L., Brown, T. M., Kjeldsen, H., McCarthy, J. K., Peri, M. L., Belmonte, J. A., Vidal, I.. Cram, L, E.. Palmer, J., Frandsen, S., Parthasarathy, M.. Petro, L., Schneider, H., Stetson, P. B. \&. Weiss, W. W.: 1993, "A search for solar-like oscillations in the stars of M67 with CCD ensemble photometry on a network of $4 \mathrm{~m}$ tclescopes", Astron. J. 106, $2441-2476$.

Gough. D. O.: 1976. "The current state of stellar mixing-length theory", in E. Spiegel \& J.-P. Zahn (eds), Problems of stellar convection, IAU Colloq. No. 38. Lecture Notes in Physics 71, Springer-Verlag, Berlin, 15-56.

Gough, D. O.: 1977a. "Random remarks on solar hydrodynamics", in R. M. Bonnet \& Ph. Delache (eds), Proc. IAU Colloq. No. 36: The energy balance and hydrodynamics of the solar chromosphere and corona, G. de Bussac, Clairmont-Ferrand, $3-36$.

(iough, D. O.: 197Tb. "Mixing-length theory for pulsating stars", Astrophys. J. $\mathbf{2 1 4}, 196-213$

Crough, D. O.: 1984a. "On the rotation of the Sun", Phil. Trans. R. Soc. London, Ser. A 313, 27 - 38.

Gough. D. O.: 1984b. "Towards a solar model", Mem. Soc. Astron. Ital. 55, $13-35$.

Gough, D. O.: 1986, "EBK quantization of stellar waves", in Y. Osaki (ed.), $H_{3}$ :drodynamic and magnetohydrodynamic problems in the Sun and stars, University of Tokyo Press, 117 - 143.

Gough, D. O.: 1990, "Comments on helioseismic inference", in Y. Osaki \& H. Shibahashi (eds), Progress of seismology of the sun and stars, Lecture Notes in Physics 367, Springer, Berlin, 283 - 318.

Gough, D. O.: 1993, "Course 7. Linear adiabatic stellar pulsation", in J.-P. Zahn \& J. Zinn-Justin (eds), Astrophysical fluid dynamics, Les Houches Session XLVII, Elsevier, Amsterdam, 399 - 560.

Gough, D. O. \& Novotny, E.: 1993, "Asteroseismic calibration of stellar clusters", in T. M. Brown (ed.), Proc. GONG 1992: Seismic investigation of the Sun and stars, ASP Conf. Ser. 42, 355 -- 357. 
Gough, D. O. \& Thompson, M. J.: 1991, "The inversion problem", in A. N. Cox, W. C. Livingston \& M. Matthews (eds), Solar interior and atmosphere, Space Science Series, University of Arizona Press, 519 - 561.

Gough, D. O. \& Toomre, J.: 1991, "Seismic observations of the solar interior", Ann. Rev. Astron. Astrophys. 29, $627-685$.

Gough, D. O. \& Vorontsov, S. V.: 1995, "Seismology of the solar envelope: measuring the acoustic phase shift generated in the outer layers", Mon. Not. R. astr. Soc. 273, $573-582$.

Gough, D. O. \& Weiss, N. O.: 1976, "The calibration of stellar convection theories", Mon. Not. R. astr. Soc. 176, $589-607$.

Grec, G., Fossat, E. \& Pomerantz, M.: 1980, "Solar oscillations: full disk observations from the geographic South Pole". Nature 288, 541 - 544.

Grec, G., Fossat, E. \& Pomerantz, M.: 1983, "Full-disk observations of solar oscillations from the geographic South Pole: latest results", Solar Phys. $\mathbf{8 2}, 55-66$.

Grevesse, N. \& Noels, A.: 1993, "Cosmic abundances of the elements", in N. Prantzos, E. Vangioni-Flam \& M. Cassé (eds), Origin and evolution of the Elements, Cambridge Univ. Press, Cambridge, 15 - 25.

Guenther, D. B.: 1989, "Age of the Sun", Astrophys. J. 339, $1156-1159$.

Guenther, D. B.: 1994, "Nonadiabatic nonradial p-mode frequencies of the standard solar model, with and without helium diffusion", Astrophys. J. 422, $400-411$.

Guenther, D. B., Pinsonneault, M. H. \& Bahcall, J. N.: 1993, "The effects of helium diffusion on solar p-mode frequencies", Astrophys. J. 418, 469 475 .

Harvey, J. W. \& Duvall, T. L.: 1984, "Observations of intermediate-degree solar oscillations", in R. K. Ulrich, J. Harvey, E. J. Rhodes Jr \& J. Toomre (eds), Solar Seismology from Space, NASA, JPL Publ. 84-84, 165 - 172.

Harvey, J. W., Hill, F., Kennedy, J. R. \& Leibacher, J.: 1993, "GONG project update", in T. M. Brown (ed.), Proc. GONG 1992: Seismic in:estigation of the Sun and stars, ASP Conf. Ser. 42, $397-409$.

Hill, H. A. \& Stebbins, R. T.: 1975, "The intrinsic visual oblateness of the Sun", Astrophys. J. 200, $471-483$.

Hill, H. A., Stebbins, R. T. \& Brown, T. M.: 1976, "Recent oblateness observations: Data, interpretation and significance for earlier work", in J. H. Sanders \& A. H. Wapstra (eds), Atomic Masses and Fundamental Constants 5, Plenum Press, $622-628$.

Houdek, G., Rogl, J., Balmforth, N. J. \& Christensen-Dalsgaard, J.: 1995, "Excitation of solarlike oscillations in main-sequence stars", in R. K. Ulrich, E. J. Rhodes Jr \& W. Däppen (eds), Proc. GONG'94: Helio- and Asteroseismology from Earth and Space, ASP Conf. Ser. 76, 528 - 531.

Hummer, D. G. \& Mihalas, D.: 1988, "The equation of state for stellar envelopes. I. An occupation probability formalism for the truncation of internal partition functions", Astrophys. J. 331, $794-814$. 
Testing a solar model: the forward problem

Iben, 1. \& Mahaffy. J.: 1976, "On the sun's acoustical spectrum", Astrophys. J. 209, L:39 - L43.

Iglesias, C. A., Rogers, F. .J. \& Wilson, B. (i.: 1992, "Spin-orbit interaction effects on the Rosseland mean opacity", Astrophys. J. 397, 717- 728.

Innis, J. L., Isaak, G. R.. Speake, C. C., Brazier, R. I. \& Williams, H. K.: 1991, "High-precision velocity observations of Procyon A - I. Search for $p$-mode oscillations from 1988. 1989 and 1990 observations", Mon. Not. R. astr. Swe. 249, 643 653.

Jcans, J. H.: 1929. Astronomy and cosmogeny. Cambridge University Press, ('ambridge (1961: Dover Publications, New York).

Jiménez, A., Pallé, P. L., Pérez, J. C.., Régulo, C., Roca Cortés, T., Isaak, G. R., Mcleod, C. P. \& van der Raay, H. B., 1988. "The solar oscillations spectrum and the solar cycle", in J. Christensen-Dalsgaard \& S. Frandsen (eds), Proc. IAI Symposium No 123 , Advances in helio- and asteroseismology, Reidel, Dordrecht, 205- 209.

Kanbur, S. M. \& Simon, N. R.: 1994, "Comparative pulsation calculations with OP and OPAL opacities", Astrophys. J. 420, $880-883$.

Kim, Y.-C.., Demarque, P. \& Guenther, D. B.: 1991. "The effect of the Mihalas, Hummer, and Däppen equation of state and the molecular opacity on the standard solar model". Astrophys. J. 378, $107-412$.

Kiriakidis, M. El Eid. M. F. \&. Glatzel, W.: 1992, "Heavy element opacities and the pulsations of 3 (ephei stars", Mon. Not. R. astr. Soc. 255, $1 \mathrm{P}-$ $5 \mathrm{P}$.

Kjeldsen, H., Bedding. T. R., Viskum, M. \& Frandsen, S.: 1995, "Solarlike oscillations in $\eta$ Boo", Astron. J. 109, $1313-1319$.

Kosovichev, A. G., Christensen-Dalsgaard, J., Däppen, W., Dziembowski, W. A., Gough, D. O. \& Thompson, M. J.: 1992, "Sources of uncertainty in direct seismological measurements of the solar helium abundance", Mon. Not. R. astr. Soc. 259, $536-558$.

Kurtz. D. W.: 1995. "Seismology and cyclic frequency variability in roAp stars", in R. K. Ulrich, E. J. Rhodes Jr \& W. Däppen (eds), Proc. GONG'94: Helio- and Astero-seismology from Earth and Space, ASP Conf. Ser. 76, $606-617$.

Kurucz, R. L.: 1991, "New opacity calculations", in L. Crivellari, I. Hubeny \& D. G. Hummer (eds), Stellar atmospheres: beyond classical models, Kluwer, Dordrecht, 441 - 448 .

Leibacher, J. and the GONG project team: 1995, "The Global Oscillation Network Group project", in R. K. Ulrich, E. J. Rhodes Jr \& W. Däppen (eds), Proc. GONG'94: Helio- and Astero-seismology from Earth and Space, ASP Conf. Ser. 76, $381-386$.

Leibacher, J. \& Stein, R. F.: 1971, "A new description of the solar five-minute oscillation", Astrophys. Lett. 7, $191-192$.

Libbrecht, K. G., Woodard, M. F. \& Kaufman, J. M.: 1990, "Frequencies of solar oscillation", Astrophys. J. Suppl. 74, 1129-1149. 
Lubow, S. H., Rhodes Jr, E. J. \& Ulrich, R. K.: 1980, "Fi e minute oscillations as a probe of the solar interior", in H. A. Hill \& W. Dziembowski (eds), Nonradial and nonlinear stellar pulsation. Lecture Notes in Physics 125, Springer-Verlag, Berlin, 300 - 306.

Lydon, T. J., Fox, P. A. \& Sofia, S.: 1992, "A formulation of convection for stellar structure and evolution calculations without the mixing-length theory approximations. I. Application to the Sun", Astrophys. J. 397, $701-716$.

Lynden-Bell, D. \& Ostriker, J. P.: 1967, "On the stability of differentially rotating bodies", Mon. Not. R. astr. Soc. 136, $293-310$.

Matthews, J. M.: 1993, "Observing the eigenmodes of $\delta$ Scuti and roAP stars", in T. M. Brown (ed.), Proc. GONG 1992: Seismic investigation of the Sun and stars, ASP Conf. Ser. 42, $303-316$.

Mihalas, D., Däppen, W. \& Hummer, D. G.: 1988, "The equation of state for stellar envelopes. II. Algorithm and selected results", Astrophys. J. 331, $815-825$.

Mihalas, D., Hummer, D. G., Mihalas, B. W. \& Däppen, W.: 1990, "The equation of state for stellar envelopes. IV. Thermodynamic quantities and selected ionization fractions for six elemental mixes", Astrophys. J. 350, 300 $-308$.

Monteiro, M. J. P. F. G., Christensen-Dalsgaard, J. \& Thompson, M. J.: 1994, "Seismic study of overshoot at the base of the solar convective envelope", Astron. Astrophys. 283, $247-262$.

Monteiro, M. J. P. F. G., Christensen-Dalsgaard, J. \& Thompson, M. J.: 1995a. "Helioseismic constraints on theories of convection", in R. K. Ulrich, E. J. Rhodes Jr \& W. Däppen (eds), Proc. GONG'94: Helio- and Asteroseismology from Earth and Space, ASP Conf. Ser. 76, 128 - 131.

Monteiro, M. J. P. F. G., Christensen-Dalsgaard, J. \& Thompson, M. J.: 1995b. "Seismic properties of the Sun's superadiabatic layer. I. Theoretical modelling and parametrization of the uncertainties", Astron. Astrophys., submitted.

Moskalik, P. \& Dziembowski, W. A.: 1992, "New opacities and the origin of the $\beta$ Cephei pulsation", Astron. Astrophys. 256, L5 - L8.

Moskalik, P., Buchler, J. R. \& Marom, A.: 1992, "Toward a resolution of the bump and beat Cepheid mass discrepancies", Astrophys. J. 385, 685-693.

Noerdlinger, P. D.: 1977, "Diffusion of helium in the Sun", Astron. Astrophys. 57, $407-415$.

Pérez Hernández, F. \& Christensen-Dalsgaard, J.: 1994a. "The phase function for stellar acoustic oscillations. II. Effects of filtering", Mon. Not. R. astr. Soc. 267, $111-124$.

Pérez Hernández, F. \& Christensen-Dalsgaard, J.: 1994b. "The phase function for stellar acoustic oscillations - III. The solar case", Mon. Not. R. astr. Soc. 269, $475-492$.

Pamyatnykh, A. A., Vorontsov, S. V. \& Däppen, W.: 1991, "A calibration of solar envelope models using the frequencies of intermediate-degree solar 
acoustic oscillations". Astron. Astrophys, 248, $263-269$.

Parker. P. D.: 1986. "Thermonuclear reactions in the solar interior", in P. A. Sturrock, T. Holzer, I). Mihalas \& R. K. (llich (eds), Physics of the Sun, nol. 1, Reidel, Dordrecht. 15 - 32.

Paterno, L., Ventura, R.. ('anuto, V. M. \& Mazzitelli, I.: 1993, "Helioseismological test of a new model for stellar convection", Astrophys. J. 402, 733 7.10 .

Petersen. J. O.: 1973, "Masses of double mode ('epheid variables determined by analysis of period ratios". Astron. Astrophys. 27,89-93.

Pidatella, R. M. \& Stix, M.: 1986. "Convective overshoot at the base of the Sun's convection zone". Astron. Astrophys. 157, $338-340$.

Pinsonneault, M. H.. Kawaler, S. D.. Sofia, S. \& Demarque, P.: 1989, "Evolulionary models of the rotating Sun", Astrophys. J. 338, 424-452.

Pottasch, E. M., Butcher, H. R. \& van Hoesel, F. H. J.: 1992, "Solar-like oscillations on $\alpha$ ('entauri $A "$, Astron. Astrophys. 264, 138-146.

Proffitt, C'. R.: 1994, "Effects of heavy-element settling on solar neutrino fluxes and interior structure". Astrophys. J. 425, $849-855$.

Proffit., ('. R. \& Michaud. G.: 1991, "Gravitational settling in solar niodels", Astrophys. J. 380, $238-250$.

Rhodes Jr, E. J., Utrich, R. K. \& Simon, G. W.: 1977, "Observations of nonradial p-mode oscillations on the Sun", Astrophys. J. 218, $901-919$.

Rogers, F. J., Swenson, F. .I. \& Iglesias, C. A.: 1995, "OPAL Equation of State Tables for Astrophysical Applications", Astrophys. J., in the press.

Rosenthal, C. S. \& Christensen-Dalsgaard, J.: 1995, "The interfacial f mode in a spherical solar model", Mon. Not. R. astr. Soc., in the press.

Rosenthal, C. S. \& Gough. D. O.: 1994, "The solar $f$-mode as an interfacial mode at the chromosphere-corona transition", Astrophys. J. 423, 488 49.5 .

Rosenthal. C. S., Christensen-Dalsgaard, J. Nordlund, §. \& Trampedach, R.: 1995a. "Convective perturbations to solar oscillations: the f mode", in V. Domingo et al. (eds), Proc. 4th SOHO Workshop: Helioseismology, ESA SP-376, ESTEC, Noordwijk, in the press.

Rosenthal, C. S., Christensen-Dalsgaard, J., Houdek, G, Monteiro, M.J.P.F.G., Nordlund, $\AA$. \& Trampedach, R.: 1995b. "Seismology of the solar surface regions", in V. Domingo et al. (eds), Proc. 4th SOHO Workshop: Helioseismology, ESA SP-376, ESTEC, Noordwijk, in the press.

Rouse, C. A.: 1977, "On the first twenty modes of radial oscillation of the 1968 non-standard solar model", Astron. Astrophys. 55, $477-480$.

Roxburgh, I. W. \& Vorontsov, S. V.: 1994, "Seismology of the solar envelope: the base of the convection zone as seen in the phase shift of acoustic waves", Mon. Not. R. astr. Soc, 268, $880-888$.

Schatzman, E., Maeder, A., Angrand, F. \& Clowinski, R.: 1981, "Stellar evolution with turbulent diffusion mixing. III. The solar model and the neutrino problem", Astron. Astrophys. 96, 1 - 16. 
Scherrer, P. H., Bogart, R. S., Bush, R. I., Hoeksema, J. T., Milford, P., Schou, J., Pope, T., Rosenberg, W., Springer, L., Tarbell, T., Title, A., Wolfson, J. \& Zayer, I.: 1995, "Status of the Solar Oscillations Investigation - Michelson Doppler Imager", in R. K. Ulrich, E. J. Rhodes Jr \& W. Däppen (eds), Proc. GONG'94: Helio- and Astero-seismology from Earth and Space, ASP Conf. Ser. 76, $402-407$.

Schiff, L. I.: 1949, Quantum Mechanics, McGraw Hill, New York.

Schmitt, J. H. M. M., Rosner, R. \& Bohn, H. U.: 1984, "The overshoot region at the bottom of the solar convection zone", Astrophys. J. 282, 316-329.

Scuflaire, R., Gabriel, M., Noels, A. \& Boury, A.: 1975, "Oscillatory periods in the Sun and theoretical models with or without mixing", Astron. Astrophys. 45, $15-18$.

Seaton, M. J., Yan, Y., Mihalas, D. \& Pradhan, A. K.: 1994, "Opacities for stellar envelopes", Mon. Not. R. astr. Soc. 266, $805-828$.

Severny, A. B., Kotov, V. A. \& Tsap, T. T.: 1976, "Observations of solar pulsations", Nature 259, 87 - 89 .

Shaviv, G. \& Salpeter, E. E.: 1973, "Convective overshooting in stellar interior models", Astrophys. J. 184, 191 - 200.

Spergel, D. N. \& Press, W. H.: 1985, "Effect of hypothetical, weakly interacting, massive particles on energy transport in the solar interior", Astrophys. J. 294, $663-673$.

Spiegel, E. A.: 1963, "A generalization of the mixing-length theory of turbulent convection", Astrophys. J. 138, $216-225$.

Steigman, G., Sarazin, C. L., Quintana, H. \& Faulkner, J.: 1978, "Dynamical interactions and astrophysical effects of stable heavy neutrinos", Astron. J. 83, 1050 - 1061.

Stein, R. F. \& Nordlund, $\AA .:$ 1989, "Topology of convection beneath the solar surface", Astrophys. J. 342, L95 - L98.

Stix, M. \& Skaley, D.: 1990, "The equation of state and the frequencies of solar p modes", Astron. Astrophys. 232, $234-238$.

Swenson, F. J., Faulkner, J., Iglesias, C. A., Rogers, F. J.\& Alexander, D. R.: 1994, "The classical Hyades lithium problem resolved?", Astrophys. J. 422, L79 - L82.

Tassoul, M.: 1980, "Asymptotic approximations for stellar nonradial pulsations", Astrophys. J. Suppl. 43, $469-490$.

Thompson, M. J.: 1988, "Evidence for a thin perturbative layer near the base of the solar convection zone", in V. Domingo \& E. J. Rolfe (eds), Seismology of the Sun 83 Sun-like Stars, ESA SP-286, 321 - 324.

Tomczyk, S., Schou, J. \& Thompson, M. J.: 1995, "Measurement of the rotation rate in the deep solar interior", Astrophys. J. Lett., in the print.

Turck-Chieze, S. \& Lopes, I.: 1993, "Toward a unified classical model of the Sun: On the sensitivity of neutrinos and helioseismology to the microscopic physics", Astrophys. J. 408, $347-36 \mathrm{~T}$.

Ulrich, R. K.: 1970, "The five-minute oscillations on the solar surface", Astro- 
phys. J. 162.993 - 1001.

Urich, R. K.: 1986. "Determination of stellar ages from asteroseismology", Astrophys. J. 306, Li3T - I,40.

Ilrich, R. h. \& Rhodes Jr. E. J.: 1977, "The sensitivity of nonradial $p$ mode eigenfrequencies to solar envelope structure", Astrophys. J. 218, 521-529.

I'mo. W.. Osaki, Y.. Anclo, H., Saio, H. \& Shibahashi, H.: 1989, Nonradial Oscillations of Stars, 2nd Edition (University of Tokyo Press).

Vorontsor. S. V.: 1988. "A search of the effects of magnetic field in the solar five-ninute oscillations", in J. Christensen-Dalsgaard \& S. Frandsen (eds), Pros. IAU Symposium No 123, Advances in helio- and asteroseismology, Reidel. Dordrecht, $151-154$.

Vorontsov, S. V.: 1991. "Asymptotic theory of acoustic oscillations of the sun and stars", Astron. Zh. 68, $808-824$ (English translation: Sov. Astron. 35, $400-408$ ).

Vorontsov, S. V. \& Shibahashi, H.: 1991, "Asymptotic inversion of the solar oscillation frequencies: sound speed in the solar interior", Publ. Astron. Soc. Japan 43. $739-753$.

Vorontsov. S. V., Baturin. V. A. \& Pamyatnykh, A. A.: 1991, "Seismological measurement of solar helium abundance", Nature 349, $49-51$.

Vorontsov, S. V., Baturin. V. A. \& Pamyatnykh, A. A.: 1992, "Seismology of the solar envelope: towards the calibration of the equation of state", Mon. Not. R. astr. Soc, 257, $32-46$.

Wambsganss, J.: 1988, "Hydrogen-helium-diffusion in solar models", Astron. Astrophys. 205, $125-128$.

Winget, D. E.: 1991. "Asteroseismology of white dwarf stars with the Whole Earth Telescope". in G. Vauclair \& E. Sion (eds), White dwarfs, Proc. 7th European workshop on white dwarfs, Toulouse, France, Kluwer, Dordrecht, $129-141$.

Xiong, D. R. \& (hen, Q. I..: 1992, "A nonlocal convection model of the solar convection zone", Astron. Astrophys. 254, 362 - 370.

Zahn, J.-P.: 1991, "Convective penetration in stellar interiors", Astron. Astrophys. 252, $179-188$.

Zahn, J.-P.: 1992, "Circulation and turbulence in rotating stars", Astron. Astrophys. 265, $115-132$. 